Rodrigo Bento Rebouças

Shear sensitive particle suspension flows in slot coating

Dissertation presented to the Programa de Pós-Graduação em Engenharia Mecânica of the Departamento de Engenharia Mecânica, PUC-Rio as partial fulfillment of the requirements for the degree of Mestre em Engenharia Mecânica.

Advisor: Prof. Márcio da Silveira Carvalho 


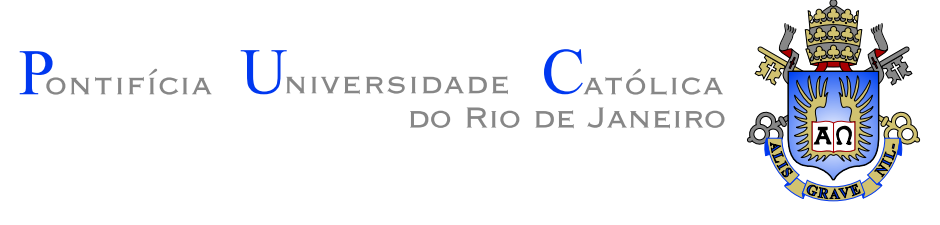

Rodrigo Bento Rebouças

\title{
Shear sensitive particle suspension flows in slot coating
}

Dissertation presented to the Programa de Pós-Graduação em Engenharia Mecânica of the Departamento de Engenharia Mecânica do Centro Técnico Científico da PUC-Rio as partial fulfillment of the requirements for the degree of Mestre.

\author{
Prof. Márcio da Silveira Carvalho \\ Advisor \\ Departamento de Engenharia Mecânica - PUC-Rio
}

Prof. Paulo Roberto de Souza Mendes

Departamento de Engenharia Mecânica - PUC-Rio

Prof. Roney Leon Thompson

Departamento de Engenharia Mecânica - COPPE/UFRJ

Prof. Márcio da Silveira Carvalho Coordinator of the Centro Técnico Científico da PUC-Rio 
All rights reserved.

\section{Rodrigo Bento Rebouças}

Rodrigo Bento Rebouças received the diploma in Mechanical Engineering from the Department of Mechanical Engineering of the University of Brasília in August 2014 with an undergraduate final project entitled $A$ study of the boundary layer equations of dilute monodisperse emulsions under the supervision of prof. Taygoara Oliveira. After that, began his master's degree at the Laboratory of Microhydrodynamics and Porous Media of the Department of Mechanical Engineering of PUCRio under the supervision of prof. Márcio Carvalho.

Bibliographic data

Rebouças, Rodrigo Bento

Shear sensitive particle suspension flows in slot coating / Rodrigo Bento Rebouças; Advisor: Márcio da Silveira Carvalho. - 2016 (em Inglês).

$104 \mathrm{f:}$ il.(color) ; 29,7 cm

Dissertação (mestrado) - Pontifícia Universidade Católica do Rio de Janeiro, Departamento de Engenharia Mecânica, 2016.

Inclui bibliografia.

1. Engenharia Mecânica - Teses. 2. Revestimento de substratos por extrusão. 3. Suspensões. 4. Líquidos Pseudoplásticos. 5. Método dos Elementos Finitos. I. Carvalho, Márcio da Silveira. II. Pontifícia Universidade Católica do Rio de Janeiro. Departamento de Engenharia Mecânica. III. Revestimento de substratos com escoamentos de suspensões pseudo-plásticas. 


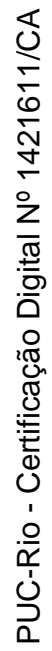

I dedicate this work to my family. 


\section{Acknowledgments}

I would like to thank, first, my dear parents and sister for the support, unconditional love and opportunity to grow in a healthy home. I can not access how I consider myself lucky to have you in my life. Love you so much!

To my girlfriend, Ranan, my great source of inspiration. Thank you for being exactly the way you are, my love. For your care, love and attention inherent of your personality. Thank you for the years we have spent together and I hope that this is just the beginning of a long journey. I love you! Also, I would like to thank Aunt $\mathrm{Su}$ for always receive me very well in your home.

To all my family and friends from Brasilia for the constant support, always very positive. Especially to my cousin Rafael for advice and help during my Doctoral application process abroad. Thank you very much, cousin.

To my friend, Ivan, for the moments of distraction, study and learning during the last years. Thanks for the academic achievements we have had together and the great opportunity to follow your constant evolution, always very sharp. I wish you all the best luck in this next step of your career towards the $\mathrm{PhD}$ and that you always keep your dream alive. Thanks a lot, my friend!

To my advisor, Prof. Marcio Carvalho, for the great opportunity to work in his research group, the classes, trips to conferences and discussions on various topics. Thank you for the attention and patience always present. Thank you very much, Professor.

To my Professor and friend, Prof. Taygoara, for leading me to a serious and committed academic life. I have great admiration for your work and conduct as a human being. My sincere acknowledgment! A special thanks to his wife, Isabel, who I had the great privilege to know better recently.

To the Professors of PUC-Rio: Paulo Roberto de Souza Mendes, Luis Fernando Azevedo, Angela Ourivio Nieckele, Ricardo Alonso and Carlos Tomei for the distinguished and valuable classes.

To the members of the examining committee of this Dissertation. In particular to Prof. Roney Leon Thompson for the very accurate and constructive comments regarding this work.

To the great Professors of UnB: Prof. Francisco Ricardo da Cunha and Prof. Gustavo Coelho Abade for the excellent lessons about the various topics of Fluid Mechanics.

To my roommates: Danilo Bona, Paula e Gustavo for the ever present company that marked my stay in Rio de Janeiro.

To all my fellow friends from the group Lmmp, in particular: Ademir, Glaucia, Nicolle, Marisa, Danmer, Jose, Ana Paula, Sergio Leal, Patrick and 
everyone else with whom I shared many laughs and knowledge during those two years.

To the staff of the department: Rosely and Roberta for the attention and help.

Finally, to the National Council of Scientific and Technological Development $(\mathrm{CNPq})$ for the scholarship and to all Brazilians that invest, through your taxes, in the promotion of serious research in many different fields. 


\section{Abstract}

Rebouças, Rodrigo Bento; Carvalho, Márcio da Silveira (Advisor). Shear sensitive particle suspension flows in slot coating. Rio de Janeiro, 2016. ए04p. MSc. Dissertation - Departamento de Engenharia Mecânica, Pontifícia Universidade Católica do Rio de Janeiro.

Coating is the main step in the manufacturing process of many common and new products such as paper, adhesive and magnetic tapes, and flexible and transparent electronics. Most of the available analyses consider the coating liquid as Newtonian. However, in many applications the coating liquid is a complex fluid composed by particles suspended in a viscous medium. The effect of suspended particles in the coating liquid on the coating flow remains unknown. Local variation of particle concentration associated with different mechanisms of particle migration may lead to viscosity gradients, which can change the flow pattern and final particle structure in the coated film. Moreover, if the particles are small enough, colloidal forces are important and hence non-Newtonian behavior such as shear-thinning and shear-thickening may occur. In this study, the conservation equations of mass, momentum and particle transport, are considered in which the viscosity is assumed to vary not only with local particle concentration but also with local shear rate (i.e. shear sensitive liquids). With this in mind, we employ the Diffusive Flux Model (DFM) to capture particle diffusion where irreversible particle-particle interactions are dominant. The conservation equations together with the set of differential equations that describes the mapping between the unknown flow domain to a fixed reference domain are solved using the Galerkin/Finite Element Method (GFEM). The resulting non-linear system of algebraic equations is solved using Newton's method coupled with a robust LU frontal solver. The results show how the rheological behavior of colloidal suspensions affects the particle distribution on the deposited liquid film and the process limits in a typical slot coating flow.

\section{Keywords}

Slot coating flows; particle suspension; shear sensitive liquids; Finite Element Method. 


\section{Resumo}

Rebouças, Rodrigo Bento; Carvalho, Márcio da Silveira. Revestimento de substratos com escoamentos de suspensões pseudo-plásticas . Rio de Janeiro, 2016. ए04p. Dissertação de Mestrado — Departamento de Engenharia Mecânica, Pontifícia Universidade Católica do Rio de Janeiro.

O processo de revestimento de substratos é uma das principais etapas na manufatura de diversos produtos, tais como eletrônicos transparentes, fitas magnéticas e adesivos. Em sua grande maioria, os estudos disponíveis na literatura consideram que o líquido de revestimento é um fluido Newtoniano. No entanto, em grande parte das aplicações, este fluido é mais complexo, sendo composto por partículas dispersas em uma fase contínua. Nesse sentido, efeitos reológicos relacionados à presença das partículas no escoamento ainda não são bem estabelecidos. Por exemplo, variações nos valores locais de taxa de cisalhamento e concentração induzem à diferentes mecanismos de migração de partículas que, por sua vez, podem mudar os padrões do escoamento e a estrutura final do filme. Além disso, se o tamanho das partículas é pequeno o suficiente de modo que forças coloidais são relevantes, fenômenos locais como redução ou aumento de viscosidade podem ocorrer dependendo das contribuições macroscópicas do escoamento. Neste trabalho, as equações de conservação de massa, momento e transporte de partículas são consideradas juntas com um modelo de viscosidade sensível a variações locais tanto de concentração quanto da taxa de cisalhamento. Nesse sentido, o Modelo de Fluxo Difusivo é empregado com o intuito de capturar fluxos difusivos de partículas em casos nos quais interações irreversíveis entre partículas são relevantes. As equações de conservação são acopladas a um sistema de equações diferencias que descreve o mapeamento numérico entre o domínio físico e outro de referência, e são discretizadas usando o Método de Elementos Finitos com a formulação fraca de Galerkin. O sistema não-linear resultante é resolvido por meio do método de Newton associado à um robusto método LU de decomposição matricial. Os resultados mostram que o comportamento reológico de suspensões coloidais afeta a distribuição final de partículas no filme depositado e os limites de operação de um processo típico de revestimento de substrato por extrusão.

\section{Palavras-chave}

Revestimento de substratos por extrusão; suspensões; líquidos pseudoplásticos; Método dos Elementos Finitos. 


\section{Contents}

1 Introduction 16

1.1 Motivation 16

$\begin{array}{lll}1.2 & \text { Slot Coating Process } & 18\end{array}$

1.3 Bibliographic Review 20

Suspension Rheology 20

Migration Mechanisms/Free Surface Flows 23

Slot Coating $\quad 25$

$\begin{array}{lll}1.4 & \text { Dissertation goals } & 27\end{array}$

1.5 Scope of the work 28

2 Mathematical formulation $\quad 29$

$\begin{array}{llr}2.1 & \text { Equations of Motion } & 29\end{array}$

Viscosity Model $\quad 32$

Boundary Conditions: Momentum Equation $\quad 35$

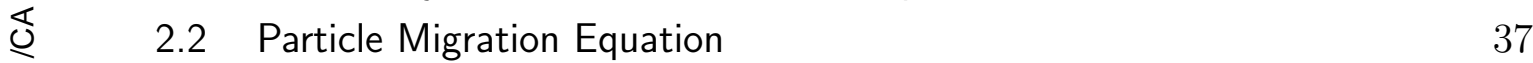

Effect of spatially varying interaction frequency 39

Effect of spatially varying viscosity 40

$\begin{array}{ll}\text { Curvature-induced migration } & 41\end{array}$

Volume fraction dependency of phenomenological parameters 42

Brownian Diffusion 43

Boundary Conditions: Diffusive Particle Iransport 46

$\begin{array}{llll}3 & \text { Numerical Methodology } & 47\end{array}$

3.1 Formulation of Free Surface Problems 48

3.2 Mesh Generation Scheme 50

Boundary Conditions: Mesh-Generation Equations 51

3.3 Interpolated Velocity Gradient 53

3.4 Weighted Residual Form 55

Expansion of the Unknown Fields 57

Element Type $\quad 58$

3.5 Solution Method 59

$\begin{array}{llll}3.6 & \text { Numerical Aspects } & 61\end{array}$

$4 \quad$ Results and discussion $\quad 62$

4.1 Numerical Validation 62

4.2 Slot Coating Analysis $\quad 67$

Mesh convergence test 68

$\begin{array}{ll}\text { Effect of shear sensitivity } & 71\end{array}$

Effects of Capillarity $\quad 80$

Low-flow limit 87 
5 Final remarks $\quad 93$

5.1 Future Works 95

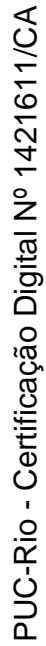




\section{List of Figures}

$1.1 \quad$ Slot coating characteristics and main components. 19

1.2 Pressure, viscous, surface tension and inertial forces must be in balance in order to sustain the flow

2.1 Cross' non-Newtonian viscosity model for a concentrated suspension at $\phi=0.30$ and particle size $a=1 \mu \mathrm{m}$. Note that $\eta_{r}=\eta / \eta_{s}$ is a dimensionless viscosity.

2.2 Boundary conditions for the conservation of momentum equations. 37

2.3 Irreversible two-body collisions: (a) constant shear rate and (b) spatially varying shear rate

2.4 Irreversible two-body collision: (a) constant viscosity and (b) spatially varying viscosity.

2.5 Direction of the migration due to the curvature of the streamline.

3.1 Mapping from the unknown physical domain to a given reference domain. The physical domain is transformed into regions geometrically simple composed by unit quadrangular domains (Adapted from Siqueira (2016)).

3.2 Stretching functions used in the calculation of the diffusion coefficients $D_{\xi}$ and $D_{\eta}$.

3.3 Boundary conditions for the equations (3.4) and (3.5) that denotes the mapping between the reference and physical domains. 53

3.4 Node representation of a typical quadrangular element where $\mathrm{B}$ and $\eta$ are local coordinates.

4.1 Flow domain of a cylindrical tube used in the numerical validation. 64

4.2 Comparison between the numerical results of the velocity profiles with Phillips' analytical solutions. The parabolic profile of a typical Poiseuille flow (i.e. $\bar{\phi}=0.0$ ) is plotted as reference.

4.3 Comparison between the numerical results of the concentration profiles with Phillips' analytical solutions.

4.4 Countors for bulk concentrations 0.40 and 0.60 , respectively. ments and 9,621 unknowns.

4.6 Representative Mesh 2 used to discretize the problem: 418 elements and 12,135 unknowns.

4.7 Representative Mesh 3 used to discretize the problem: 608 elements and 17,405 unknowns.

4.8 Predictions using three different meshes of the particle concentration profile at the exit of the deposited film.

4.9 Contour of particle concentration for a suspension in which the viscosity is a constant function of the bulk concentration, $\bar{\phi}=0.30$. 72

4.10 Contour map of particle concentration of the upstream region considering migration Model I and the viscosity model of Krieger. 
4.11 Contour map of particle concentration of the upstream region considering migration ModeI II and the viscosity model of Krieger. 74

4.12 Contour map of particle concentration of the upstream region considering migration Model III and the viscosity model of Krieger. 74

4.13 Particle concentration profiles the deposited film (i.e., $X=1.7$ $\mathrm{mm}$ ) for a viscosity model $\eta=\eta(\phi)$ and for migration Models $\mathrm{T}$, III and III

4.14 Graphs of the position and shape of the upstream meniscus when $\phi=0.30$ : (a) using Krieger's viscosity model and (b) using Cross' model

4.15 Contour map of particle concentration considering migration Model I and the viscosity model of Cross.

4.16 Contour map of particle concentration considering migration Model II and the viscosity model of Cross.

4.17 Contour map of particle concentration considering migration Model III and the viscosity model of Cross.

4.18 Particle concentration profiles at the final section of the deposited film (i.e., $X=1.7 \mathrm{~mm}$ ) for a suspension in which the viscosity is a function of local particle concentration only, $\eta=\eta(\phi, \dot{\gamma})$ and for Models I, II and III.

4.19 Contours of particle concentration for Capillary number 0.077 using Krieger's viscosity model and migration ModelT. 81

4.20 Contours of particle concentration for Capillary number 0.077 using Cross' viscosity model and migration Model III.

4.21 Contours of particle concentration for Capillary number 0.386 using Cross' viscosity model and migration Model II.

4.22 Contours of particle concentration for Capillary number 1.158 using Cross' viscosity model and migration Model II.

4.23 Scheme of the Couette and Poiseuille contributions at the downstream region of a slot coating flow.

4.24 Contours of particle concentration for Capillary number $\mathrm{Ca}=$ 0.386: (a) for a final film thickness $t=H / 2$ (b) for a final film thickness $t=7 \mathrm{H} / 20$.

4.25 Profiles of particle concentration and upstream meniscus (a) and (b), respectively, for a Capillary number $\mathrm{Ca}=0.077$.

4.26 Profiles of particle concentration and upstream meniscus (a) and (b), respectively, for a Capillary number $\mathrm{Ca}=0.386$

4.27 Profiles of particle concentration and upstream meniscus (a) and (b), respectively, for a Capillary number $C a=1.158$

4.28 Coating window of a slot coating process as a function of the pressure difference $\left(P_{a t m}-P_{v a c}\right)$, fixed Capillary number $C a$ and gap-top-thickness ratio $H / t$.

4.29 Low-flow limit predictions by: viscocapillary model and viscosity models of Krieger and Cross considering migration Model T.

4.30 Downstream free surface at different gap-to-thickness ratio and Capillary numbers considering concentration-dependent only viscosity. 
4.31 Contour map of concentration field considering viscosity model of Cross' considering a fixed Capillary number $\mathrm{Ca}=0.2316$.

4.32 Particle concentration profiles comparing both viscosity models considering a fixed Capillary number $\mathrm{C} a=0.2316$.

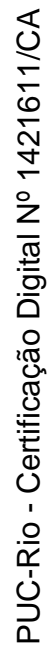




\section{List of Tables}

3.1 Table with different combination of basis functions $\varphi$ and $\psi / \chi \quad 58$

3.2 Relationship between local numbering and unknown coefficients degrees of freedom in each element. 59

$4.1 \quad$ Number of elements in each region of Mesh 1. $\quad 69$

4.2 Number of elements in each region of Mesh 2.

4.3 Number of elements in each region of Mesh 3.

4.4 Minimum and maximum local concentrations for each mesh. 70

4.5 X-coordinate of the dynamic contact line related to migration models I, II and III.

4.6 Capillary numbers and adjustable parameters for a stable slot coating flow considering three cases. $\quad 81$

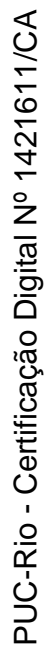




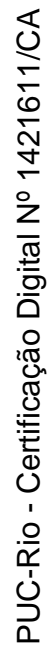

"Although no one can go back and make a new start, anyone can start now and make a new ending".

Chico Xavier. 


\section{Introduction}

\section{1}

\section{Motivation}

Coating is an industrial manufacturing process where a gas medium in contact with a solid surface is replaced by a layer of a liquid that, afterwards, is cured or solidified. Usually the coating layer must be uniform, thin, continuous and smooth in order to meet technological and industrial specifications. This kind of process can be found in the manufacturing process of many common and old products such as paper, adhesive and magnetic tapes. Moreover, plenty of modern devices, such as flexible and transparent electronics and displays like Plasma, LCD ${ }^{m}$, OLED $^{\boldsymbol{\square}}$ and so forth, are manufactured using coating technology.

There is a wide range of coating processes available in industry, such as roll, blade/knife, curtain, dip and slot coating. Essentially, the final film thickness and its micro or nano structure need to be controlled and are directly related to the quality of the coated product, whether optically, photochemically, electronically or mechanically. In many coating applications, the coating liquid is a complex fluid composed by particles suspended in a viscous medium. As a consequence, in an effort to better understand and monitor the aspects of the deposited film, a complete flow model of these complex fluid is needed.

The rheology of hard or smooth particles suspended in a viscous fluid is a topic in constant development in order to meet many scientific and industrial requirements. In many biological systems (e.g. bloods), engineering applications and industrial processing, the properties of a fluid in which small particles or corpuscles are suspended and convected by the motion of the fluid are of great concern. This type of complex fluid is known in the literature as dispersion.

\footnotetext{
${ }^{1}$ Liquid Crystal Display

${ }^{2}$ Organic Light-Emitting Diode
} 
In general, a dispersion can be subdivided into three main categories. Firstly, if the suspended medium is composed by solid particles, then the dispersion is classified as a suspension. Second, one can find dispersions classified as emulsions which are liquid droplets immersed in another liquid and, finally, if the suspending medium is a gas, then the given denomination for the dispersion is foam (Macosko, 1994; Larson, 1999.9). A special attention will be given to the characteristics and rheology of suspensions, as this subcategory is closely linked to the main application of this work, which is slot coating.

According to the size and distribution of particles, suspensions can be classified in different ways. Suspensions are mono-dispersed if all the particles have the same size (given by their radius $a$, for spherical particles); and polydispersed if the suspension presents a distribution of sizes over the continuous phase. Moreover, suspensions with particle size: $a<1 \mathrm{~nm}$ are considered homogeneous solutions; $1 \mathrm{~nm}<a<1 \mu \mathrm{m}$ are colloidal suspensions; $a>1 \mu \mathrm{m}$ are non-colloidal suspensions ([Dbouk, [201]).

The presence of the particles changes the material properties of the suspension and, especially, the viscosity of the bulk fluid is affected. This distinct behavior is closely related to inter-particle and particle-fluid interactions that are, consequently, influenced by the volume fraction of particles, $\phi$. According to the volume concentration, a suspension is considered diluted if $\phi<0.05$, semi-diluted if $0.15<\phi<0.30$ and concentrated for higher values in concen-

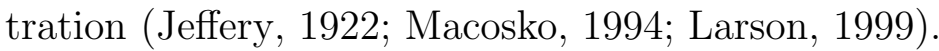

In the scale of the particles, electrostatic (repulsion), van der Waals (attraction) and Brownian (thermal) forces coexist and are in balance with the hydrodynamic forces acting over them as a result of the imposed flow. The magnitude of these colloidal and Brownian forces will depend on the size and shape of the particles. For instance, for concentrated colloidal suspensions where Brownian motion dominates, non-Newtonian behavior such as shearthinning and shear-thickening may occur. On the other hand, if the particles are big enough (non-colloidal suspensions), Brownian motion can be neglected and the suspension can be considered a Newtonian fluid with viscosity varying only as a function of local particle concentration.

All of these rheological characteristics are of great importance in an effort to define the optimum set of operating parameters that will deliver the best final product possible in a coating process. In general, as the deposition of the liquid layer is in course, the particles inside the suspending medium may migrate in response to gradients in concentration and shear rate. Other types of migration mechanisms may also occur due to sedimentation, curvature and thermal agitation. The main purpose of this work is to study how rheological 
behavior of suspensions and particle migration can influence the quality of the final film delivered as a result of a slot coating manufacturing operation.

\section{2}

\section{Slot Coating Process}

This method, as other coating processes, employs a chamber-and-slot die configuration that drives the coating liquid towards the surface to be coated and meters the flow. A typical slot coating apparatus and its most important components are shown in Figure $\mathbb{L}$. In this method it is relevant to emphasize that the gap between the slot die and the moving substrate is set as one of the process parameters. Also, slot coating is a type of pre-metered process in which the thickness of the deposited liquid layer is set a priori by the flow rate fed into the coating die (through the feed slot) and the substrate velocity or web speed. In fact, the film thickness is directly proportional to the flow rate, thus, the greater the flow rate, the thicker is the final film, for a fixed web speed.

Provided that a liquid film layer is deposited onto a solid substrate, two distinct free menisci are formed. Downstream is the free surface of the deposited film that extends itself in the upstream direction to a contact line where the flowing liquid departs from the solid wall. On the upstream side, the other free meniscus bounds the region between the moving substrate, a moving wetting line where the liquid touches the solid surface and another contact line where the liquid departs from the upstream die. Higgins \& Scriven (11980) defined the region between these two menisci, regardless how far apart they are, as the coating bead.

The flow uniformity in the bead region is also strongly affected by parameters like the die configuration (i.e. lip design and gap profile), the size of the gap, web speed and rheological properties of the liquid. Essentially, the longer the coating bead, the greater is the ability to overcome variations in the the gap width (re-metering) due to, for instance, local changes in the topography of the substrate. For viscous liquids, imposing a pressure difference across the bead is a useful approach in order to maintain this layer of fluid as stable as possible (Beguin, 1954). This is usually accomplished by using an upstream vacuum chamber so that a constant pressure less than atmospheric can be maintained and creates an adverse pressure gradient necessary in the case of thin liquid layers. This Poiseuille flow combines with a downstream Couette or viscous flow, due to the drag of the substrate, and delivers the velocity profile under the upstream and downstream dies. The use of a vacuum chamber often broadens the range of thicknesses given for a specific gap. 


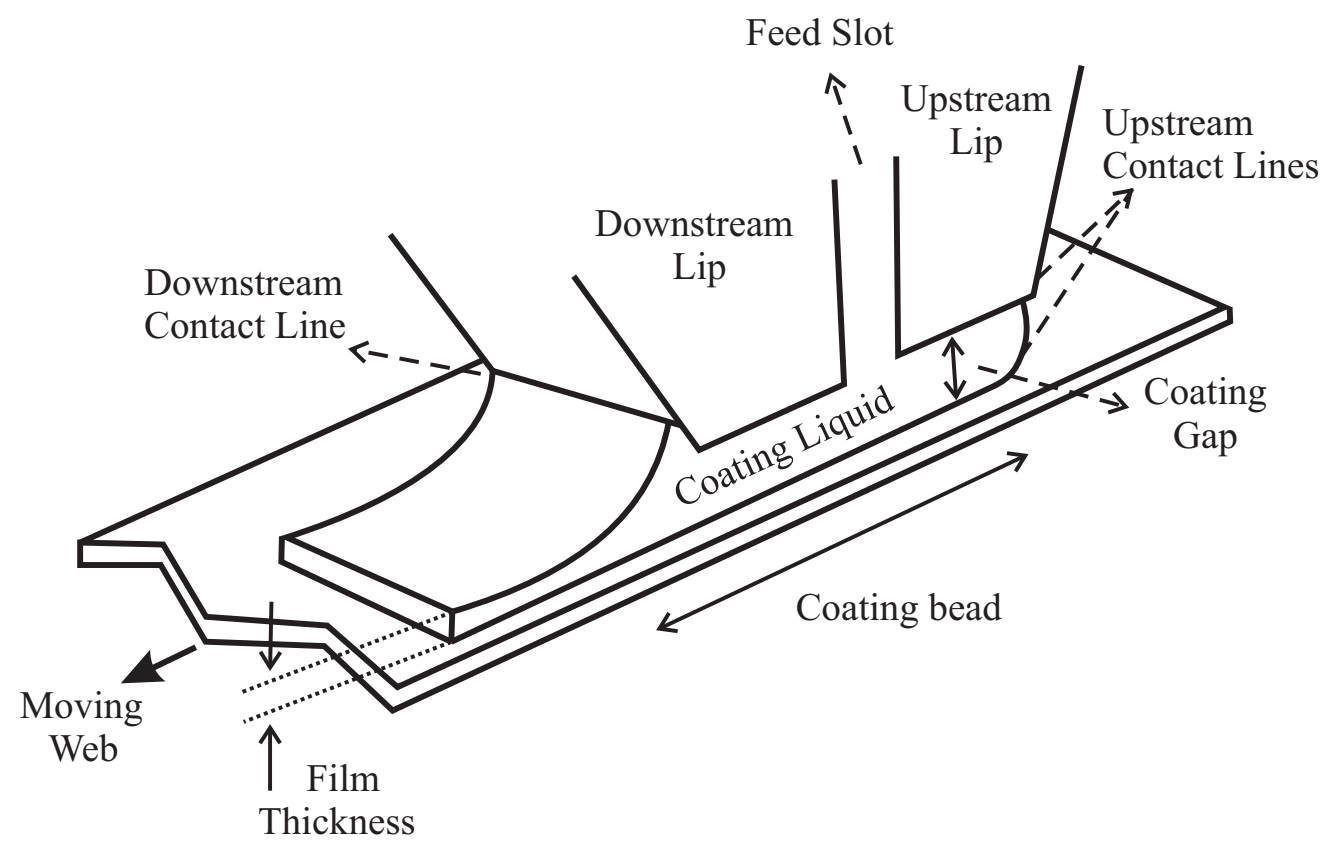

Figure 1.1: Slot coating characteristics and main components.

These operating conditions control the volume of the coating bead, the attachment of both separation lines (upstream and downstream), the occurrence of recirculating flow (microvortices) inside the bead and the generation of instabilities such as bead break-up or rivulets and down-web ribbing. The main objective of the process is to determine and control all the operating conditions in order to attain a stable, steady state and two-dimensional flow. Explanations for this controlled stability lay on the forces acting upon the flow, in a way that, pressure, viscous, surface tension and inertial forces must be in balance. If those forces are not in balance, coating bead will break into a 3-D (i.e. rivulets and ribbing) or transient flow. The set of all these operating parameters that delivers a uniform and steady deposited film defines the widespread concept of coating window. Coating windows can be constructed from careful experimentation or from theoretical modelling of the coating flow. Usually, the experimental approach is considerably expensive. On the other hand, trustful theoretical models can be applied to analyse the quality of a coating process over large ranges of variables at significantly low cost. In any case, theoretical modelling predictions should be compared with experimental data by means of validation (Carvalho \& Kheshgi, 20(0) 


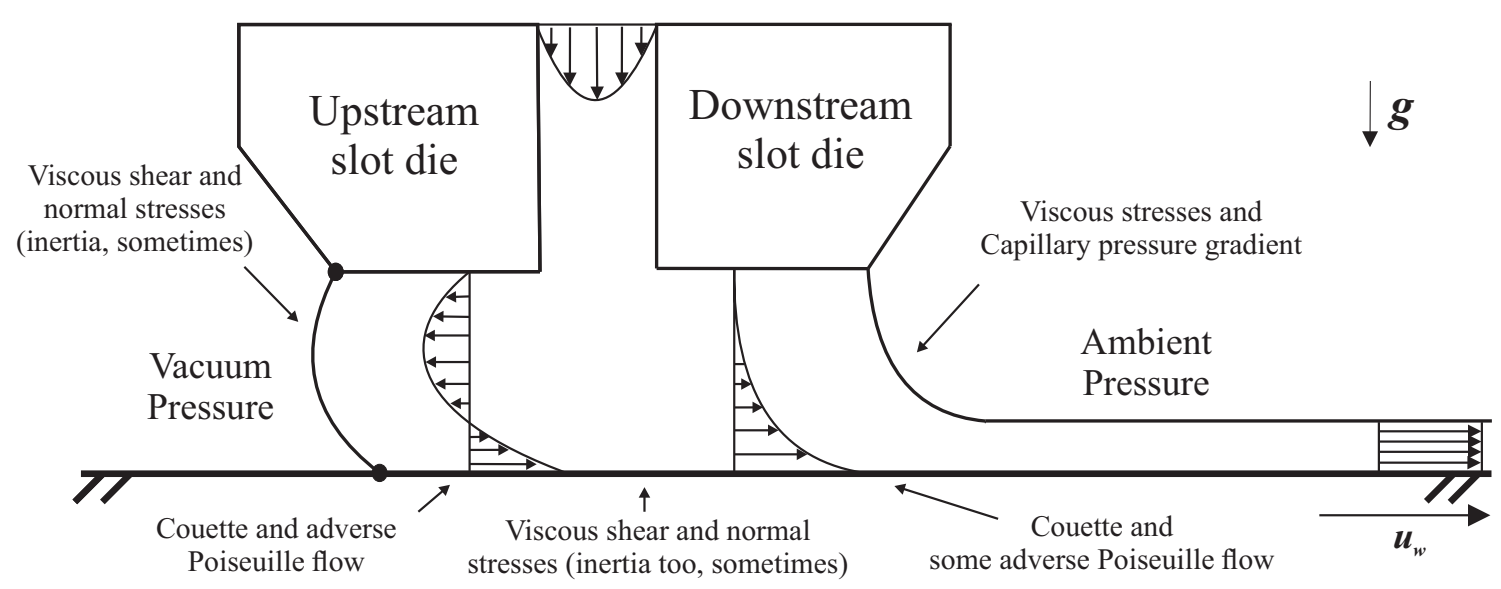

Figure 1.2: Pressure, viscous, surface tension and inertial forces must be in balance in order to sustain the flow.

\section{3}

\section{Bibliographic Review}

As this work is concerned with shear sensitive flows of particle suspensions, specifically spherical particle suspensions, this section will present a literature review on the rheology of suspensions taking into account particle migration mechanisms in free surface flows such as those found in slot coating operations. This section is subdivided into three main bibliographic reviews: first, on the viscosity of non-colloidal and colloidal suspensions; second, on the developments of particle migration mechanisms directed to free surface flows; and, finally, on the most important studies regarding, mainly, slot coating processes and operating limits.

\subsection{1}

\section{Suspension Rheology}

Investigations on suspension's rheology date from the early $1900 s$ when Einstein (1906, 1917) first calculated the effective viscosity, $\eta_{r}$, of a very dilute suspension of spherical rigid particles. Here, the effective viscosity of a suspension is defined as the ratio between the viscosities of the suspension, $\eta$, and of the suspending medium, $\eta_{s}$, respectively. In this case, Einstein suggested that adding particles into the suspending medium increases its viscosity by thermal dissipation, so that, the viscosity is a function of the particle volume concentration, $\phi$. Likewise, Jeffery (1.922) extended the latter work to the case of particles of ellipsoidal shape. Both authors established a linear relationship between the viscosity of the suspension and the volumetric concentration of particles given by 


$$
\eta=\eta_{s}(1+K \phi)
$$

where $K$ is a factor that assumes the value of 2.5 for rigid spheres and is a function of the axial ratio for ellipsoids. Commonly, for elongated particles, it is found that $K>2.5$, although for soft spherical particles the values of $K$ can decay below 2.5 (Vand, 1.947). It is important to highlight that Einstein's viscosity equation is valid for very dilute suspensions with a particle volume fraction below 0.05. It is remarkable that Eq. (ㅁ. $)$ does not take into account hydrodynamic interactions between particles. For a slightly higher volume fraction $(\phi<0.15)$, in a semi-dilute regime, this inter-particle interactions start to be relevant for the computation of the relative viscosity. Batchelor and co-workers proposed a model in which pair interactions between particles are regarded by a second order term in concentration, $\phi^{2}$ (Batchelor \& Green, 1.972; Batchelor, 1.977).

A considerable amount of work has been done in order to predict the viscosity of dilute suspensions of spherical particles. However, it is common sense that in most industrial applications, the suspensions have a higher order of concentration so that research addressed to this area has been also of great importance (Barnes et al., 1.98.9).

At phase volume greater than 0.3 , out of the semi-dilute regime, multibody interactions between particles become important and empirical models were first developed by Guth \& Simha (11936); Vand (11947); Simha (1949). For instance, Simha (1.94.9) proposed an empirical equation in which the dependance of the viscosity on the concentration can be expressed as a power series of higher order of $\phi$. Later on, Eilers (1947) and Mooney (19.5T) presented a work on the viscosity of concentrated suspensions of rigid and spherical particles. The latter author evolved an analysis that considered the space-crowding effect of the suspended spheres on each other in such a way that the interaction parameters were determined experimentally. This limiting concentration of particles is known as the maximum crowding factor or packing fraction, $\phi_{m}$, and this parameter ranges from around 0.5 to 0.75 (Barnes et al,, 1989).

Thereafter, Krieger \& Dougherty (195.9) modified the logarithmic equation presented by Mooney (1951) in an effort to obtain an excellent fit over their entire range of concentration reports. The resulting relation is well-known as Krieger-Dougherty equation,

$$
\eta=\eta_{s}\left(1-\phi / \phi_{m}\right)^{-[\eta] \phi_{m}}
$$


where $[\eta]$ is the intrinsic viscosity of the suspension. Equation (ㄹ) is highly cited in the literature and has been the basis for the determination of the viscosity of suspensions in both Newtonian plateaus at low and high shear rate, $\eta_{0}$ and $\eta_{\infty}$, respectively. Indeed, similar studies were performed by other authors in which the fitting parameters, $[\eta]$ and $\phi_{m}$, were modified in order to adjust the model with existing experimental data (Maron \& Pierce, 1956; Quemada, 1977; de Kruif et al, 1985; Choi \& Krieger, 14986; Shewan \& Stokes, 2015).

Up until this point, the analysis has been concentrated on suspensions of the type viscosity/phase volume relationships that agree considerably well with experimental data at very low and high values of shear rates. Nonetheless, experimentation has revealed that a general viscosity curve, as a function of shear rate or dimensionless shear stress, for all types of suspensions will also present regions of shear thinning and possible shear thickening. This nonNewtonian behavior depend not only on the magnitude of the forces acting in the microstructure, but also on the microstructure itself (Laun, 1984; Barnes et al, 1989; Stickel \& Powell, 2005; Hinch, 2011).

A notable number of researchers have investigated the viscosity of suspensions including the whole spectrum of shear rates, concentrations and particle sizes. As a consequence, empirical models have been developed in order to capture these zones of Newtonian and non-Newtonian behavior previously mentioned. In this manner, Cross (1970) derived an equation to evaluate the viscosity of pseudo-plastic, i.e., shear-thinning fluids. This equation depends on the low- and high-shear viscosities at a given concentration, on the microstructure network and on the shear rate imposed by the flow. A few years later, Carrean (1972) proposed an equivalent model in which both cases, Cross' and Carreau's relations, the shear rate was used as an independent variable. Alternatively, a similar model, known as Ellis model (Bird et al, ए987; Barnes et al, 1498.9), based on an analysis done by Krieger (1972), applied a modified Peclet number as an independent variable. Albeit these approaches seek to predict pseudo-plastic behavior, other works have been done in an effort to capture shear thickening, normal stress differences and self diffusion patterns as well (Sato, 1995; Foss \& Brady, 2000; Cheng et al., 2011). For instance, shear thickening may appear at high shear rates elucidating the transition from a non-frictional rheology, where the microstructure is well defined by layers of continuous fluid and lanes of particles, to another determined by frictional contacts (Seto et al, 2013; Wyart \& Cates, 2014). As a matter of fact, computer simulations have helped to predict suspension mechanics and rheology. Among these simulation methods one can identify simulations applying 
Stokesian dynamics, dissipative particle dynamics and the lattice Boltzmann method (Bossis \& Brady, 1.98.9; Sato, 11997; Hill et al., 2001).

\subsection{2}

\section{Migration Mechanisms/Free Surface Flows}

With regards to particle migration mechanisms, the first experimental evidence was presented by Karnis et al. (1966). In this work the authors identified that the velocity profiles of spheres, rods, and discs suspensions in a tube flow assumed a blunted delineation in the center line as the concentration increased. This deviation from the parabolic distribution of velocities was shown to result from a migration of particles towards the center of the tube due to wall effects firstly described by Vand (11947). Similar measurements were also performed in a Couette flow, but still, this novel phenomenon was not fully understood.

Pointing to this end, Eckestein et al. (11977) presented a study in which a self-diffusion coefficient, $\mathcal{D}$, was determined using a concentric-cylinder Couette apparatus to account for particle migration in suspensions of spherical and disk-like particles. In this sense, the authors had found values of $\mathcal{D}$ proportional to $a^{2} \dot{\gamma}$ for suspensions with low concentration $(0<\phi<0.2)$. Three years later, Gadala-Maria \& Acrivos (11980), also using a Couette device, verified that the torque signal and hence, the viscosity of the suspension, decreased with the time of shearing and eventually reached an equilibrium value for concentrations higher than 0.3. Later on, Leighton \& Acrivos (1987a,b) suggested that the phenomenon observed by Gadala-Maria \& Acrivos ([1980) was intrinsically related to the arrangement assumed by the particles, in response to a shearing flow input, due to shear-induced migration out of the Couette gap and into the fluid reservoir, which decreases particle concentration in the gap and, therefore, the measured viscosity. Their theoretical analysis and experimental evidence also showed that this shear-induced migration arises from gradients in concentration and shear stress as a result of irreversible two-body collisions between particles in concentrated suspensions. Further discussion around this topic will be held on the following chapters of the present work.

With this in mind, Abbott et al. (1.991) used Nuclear Magnetic Resonance (NMR) to evaluate the dependence of shear-induced migration of particles on the evolution of particle concentration and velocity profiles. In the same path, Phillips et al. (1992) used a modified version of Leighton and Acrivos' diffusive flux expressions to generate a constitutive equation that accounts for flow-induced particle migration. Therefore, two adjustable constants arose in this relation, one associated to a gradients in shear rate, $k_{c}$, and the other to a gradients in viscosity, $k_{\eta}$. These constants are $\mathcal{O}(1)$ and are evaluated by 
velocity and concentration profiles measured experimentally by using NMR in a Couette device (Majors et al., 1989). This equation is known in the literature as the Diffusive Flux Model or DFM. Similar studies using this model to examine a Couette flow have been performed with finite volume and finite element methods in order to capture shear-induced migration in viscous flows (Zhang \&. Acrivos, 11994; Fang \& Phan-Thien, 19995). Krishnan et al. (11995) apud Subia et al. (1998) proposed adaptations to the DFM which incorporated effects of curvature in accordance to experimental observations made by chow et al. (11994) in parallel plate flows using NMR. Subia et aL. (11998), in turn, integrated the model of Phillips et al. (11992) into a Finite Element Method (FEM) context and verified that the model was consistent in capturing essential features of different flow problems, comparing their numerical results with NMR imaging experiments. In the same year, Graham et al. (1998) used suspension rheometry to observe that an additional source of particle migration, rather then surface roughness, may lead inter-particle interactions. In this sense, they proposed that the phenomenological parameters of the DFM should also vary with local particle concentration. Later on, Tetlow et al. (11998) used experimental data to investigate the latter approach and showed that the ratio $k_{c} / k_{\eta}$ can be modeled as a linear function of the local particle concentration. With this in mind, Kim et al. (2008) gathered all those variations of the DFM and studied the influence of considering curvature-induced migration and volume fraction dependent parameters. More recently, Rebouças et al. (2016) analyzed the fully-developed flow of particle suspensions in a tube flows using the original DFM to evaluate the effect of both particle migration and shear-dependent viscosity.

Another approach in an effort to study particle migration, acknowledged as the Suspension Balance Model (SBM) (Jenkins \& McTigue, 1990); Nott \& Brady, 1994; Morris \& Boulay, 1.999), was presented to explain that particle migration is due to the divergence of the normal components of the particle phase stress. More recently, Snook et al. (2016) have employed this method to study shear-induced migration in oscillatory pipe flow comparing their results with realistic rheological laws. The main difference between these two models is that the DFM brings a more phenomenological analysis and gives a better insight on the fundamental physics of the problem. In this work, the Diffusive Flux Model was chosen because of its relative simplicity, good accuracy and lower computational cost.

Since the seminal report released by Gadala-Maria \& Acrivos (1980); Leighton \& Acrivos (1987a,, $\mathrm{b})$; Phillips et al. (11992), there have been a large body of studies on migration mechanisms of particles in different types of 
geometries, however, focusing mostly on flows bounded by solid walls. Based on this, it is believed that the first experimental attempt to describe migration of particles in an unbounded, i.e., free-surface flow has been done by Hushand et al. (19.94). In this research the authors analyzed the shear-induced particle migration in a bimodal concentrated suspension flowing in a rectangular channel held in an inclined base. Afterwards, Tirumkudulu et al. (19.99, 2000) observed experimentally that the particles tend to accumulate in bands along the axis of a partially filled rotating horizontal cylinder. Timberlake \& Morris (20102), in turn, dealt with the dynamics of this band formation, but now, using a partially filled Couette apparatus and imaging analysis techniques. Further analysis on shear-induced migration aiming free-surface flows were also exploited by Loimer et al. (2002); Singh et al. (2006); Min \& Kim (2010). As matter of fact, Min \& Kim (2010) verified that even though the downstream velocity profile for a slot coating application is fully developed and flat, the particle concentration never reaches a uniform distribution. Furthermore, they also pointed out to the fact that scarcely studies have been done so far in order to precisely measure or estimate the particle concentration profile near the free surface boundary. Recent advances in this field have been made by Silva (201:3) and Araújo (2014) where particle concentration profiles were computed considering the suspension as a Newtonian fluid with a concentration dependent viscosity. In addition to that, Siqueira (2016) enlarged the latter works to investigate slot coating flows of suspensions of elliptical particles. The main results aim to investigate the effects of typical operating parameters on particle orientation and distribution.

As the scope of this work is related to the shear-sensitivity of particle suspension flows in a slot coating precess, it will be presented in the next section a brief review on this industrial application. Indeed, a typical slot coating flow combines aspects of the rheology of suspension, such as local variations in viscosity, linked to mechanisms of particle migration in free-surface flows due to gradients in concentration and shear rate.

\subsection{3}

\section{Slot Coating}

One of the first attempts to evaluate the film thickness of a coating process can be attributed to Landau \& Levich (1942). In this paper, the authors tackled the problem of depositing a thin layer of liquid upon a surface of a solid that is submerged and pulled out of a coating liquid vessel, i.e., dip coater. The main idea was to determine the thickness of the dragged liquid layer with respect to the speed of the fluid and its physical properties such 
as its viscosity, surface tension and density. The model treated the flow in the thin film as one-dimensional and as a function of small Capillary and Reynolds numbers, $C a$ and $R e$, respectively. The Capillary number expresses a balance between viscous and interfacial forces and is defined by $C a=\eta V / \sigma$, where $V$ is the substrate's velocity, $\eta$ is the viscosity of the coating liquid and $\sigma$ is the interfacial tension. On the other hand, the Reynolds number defines a ratio between inertial and viscous contributions and is expressed by $R e=\rho V H / \eta$, where $\rho$ is the liquid density and $H$ is a characteristic length of the flow. In slot coating process, the film thickness is prescribed by the flow rate fed into the coating die and the web speed. In this case, the flow rate is independent of liquid properties and slot coating is considered a pre-metered method. Later on, Ruschak (11976) used a singular perturbation method in coating flows dominated by surface tension to extend the work performed by Landau \& Levich (1.942) and demonstrated that the pressure drop across the downstream region is a function of $1.34 \mathrm{Ca}^{2 / 3} \sigma / t$, where $\mathrm{t}$ is the final film thickness. The former author assumed that the flow rate was low enough so that viscous effects were negligible within the coating bead and hence capillary pressure alone dictates the process operability limits.

Still in this track, Higgins \& Scriven (1980) defined a region of flowing liquid between the gas-liquid surfaces, regardless how far apart they are, as the coating bead in an effort to explain the existence of the so called coating window. In this manner, the authors approximated the flow in this region as a sum of lubrication flow regimes: one, upstream of the feed slot where a Poiseuille flow exactly counters a Couette flow in order to guarantee the stability of the upstream meniscus; other, downstream of the feed slot where there is a Poiseuille contribution that either adds or subtracts from the Couette induced flow so that the net flow is equal to the prescribed value into the coating die. From the results of the viscocapillary model, the authors concluded that, in many applications, the viscous effects due to viscous drag of the moving substrate are also important in settling the bounds of a coating window as well as to control of the vacuum pressure and web speed. Henceforth, additional theoretical and experimental efforts were made in order to determine the limits of operability and flow stability (Sartor, ए990); Gates, 11996).

Deryagin \& Levi (1964) were the first to point out a more important limit, in terms of industrial applications, which is known in the literature as low-flow limit. This concept is related to the maximum web speed possible for a fixed film thickness, or the minimum film thickness at a given substrate velocity, at which the coating bead is stable. For instance, as the gap width is fixed in a slot coating operation, the flow rate under the downstream die, 
due to the viscous drag of the substrate, is constant. Therefore, if the film thickness and, consequently, the flow rate in the feed slot decreases then the adverse contribution from the Poiseuille flow must increase in order to meet this reduction in flow rate under the light of mass conservation principles. The minimum flow rate possible (and hence the minimum film thickness) is determined by the maximum pressure gradient attainable at the downstream meniscus. Further studies in this area were performed by Lee \& Lin (1992) and Carvalho \& Kheshgi (2000). The latter authors showed that low-flow limit in cases of high Capillary and Reynolds numbers varies significantly from those cases of lower ones. They found that at large Capillary and Reynolds numbers, the coating window is broadened and also the range of applicability of the coating operation. Still in this path, Romero et al. (2004) analysed the lowflow limit in the slot coating flow of a non-Newtonian liquid both by theoretical and experimental approaches. A few years later, Lin et al. (2010) applied a flow visualization technique to study the positions and shapes of the liquid-gas interfaces just before and after coating failure and compared their experimental results with theoretical predictions.

Recently, Maza \& (arvalho (2015) extended the work done by Romero $\&$ Carvalho (20108) to determine the amplitude of oscillation of each coated layer in a two-layer slot coating process. The results showed that variations of each layer can be minimized by changing geometrical and physical parameters of the flow. In terms of particle migration mechanisms, as stated earlier in this work, Min \& Kim (2010), Silva (2013), Araújo (2014) and Siqueira (2016) presented novel results indicating that the particle concentration profile in the final coated film is not uniform.

\section{4 \\ Dissertation goals}

Most available studies on slot coating consider the coating liquid as a Newtonian fluid. In the case of particle suspensions, the available models are restricted to non-colloidal suspensions, at which the local viscosity is a function of the local particle concentration and independent of the shear rate. However, depending on particle size, inter-particle forces may balance with macroscopic forces so that non-Newtonian behavior may appear. In this sense, the main goal of this work is to develop a mathematical model to describe slot coating flows of spherical particle suspensions that takes into account particle transport mechanisms and also shear-sensitive behavior. In fact, the main focus is to apply this model to study the effect of liquid rheological properties 
on processes limits and particle distribution in the coated film. With this in mind, we employ the Diffusive Flux Model (DFM) with a few adaptations to capture particle diffusion and the viscosity model of Cross (1970) to express the shear-rate dependency. The final set of coupled differential equations is solved numerically by using Galerkin/Finite Element Method (GFEM). The results show the rheological behavior of shear-sensitive particle suspension on particle distribution and on operating limits of a typical slot coating application.

\section{5}

\section{Scope of the work}

This dissertation is organized as follows. The mathematical formulation of the problem is presented in Chapter $\S 2$. Chapter $\S 3$ describes the numerical methodology and issues related to this work. In addition to that, Chapter $\S 4$ shows the main results and discussions of this dissertation. To sum up, Chapter $\S 5$ presents a brief conclusion and sheds light to a few important future works. 


\section{2 \\ Mathematical formulation}

\section{1}

\section{Equations of Motion}

The physical laws that govern the motion and transfer of momentum between particles immersed in a liquid medium are Newton's second law of motion and the mass conservation principle. The application of those laws in an infinitesimal control volume of a given fluid gives birth to the mass-conservation and Navier-Stokes equations, respectively. For incompressible fluids, they are:

$$
\nabla \cdot \boldsymbol{u}=0
$$

$$
\rho \frac{D \boldsymbol{u}}{D t}=\nabla \cdot \boldsymbol{\sigma}+\rho \boldsymbol{g}
$$

where $\rho$ is density of the fluid, $\boldsymbol{u}$ is the Eulerian velocity vector, $\boldsymbol{\sigma}$ is the stress tensor (Cauchy stress tensor) and $\mathbf{g}$ represents a body force per unit of mass due to the action of the earth's gravitational field. In Eqs. (2.]) and ([2.2) the notation $D / D t=\partial / \partial t+\mathbf{u} \cdot \nabla$ is said to give a time derivative following the motion of the fluid, or a material derivative. This operator is meaningful only when applied to a field that varies as a function of time $t$ and position $\boldsymbol{x}$, such as, the velocity field (Batchelor, 1967).

Considering the following characteristic scales of length, velocity and time: $H, V$ and $H / V$ and, also, assuming that the stress tensor scales with $\eta V / H$, Eq. (2.T2) can be written in a dimensionless form as

$$
R e\left(\frac{\partial \widetilde{\boldsymbol{u}}}{\partial \widetilde{t}}+\widetilde{\boldsymbol{u}} \cdot \widetilde{\nabla} \widetilde{\boldsymbol{u}}\right)=\widetilde{\nabla} \cdot \widetilde{\boldsymbol{\sigma}}+S t \widetilde{\boldsymbol{g}}
$$

where 


$$
\widetilde{\boldsymbol{x}}=\frac{\boldsymbol{x}}{H}, \quad \widetilde{t}=\frac{t}{H / V}, \quad \widetilde{\nabla}=H \nabla, \quad \widetilde{\boldsymbol{u}}=\frac{\boldsymbol{u}}{V}, \quad \widetilde{\boldsymbol{\sigma}}=\frac{\boldsymbol{\sigma}}{\eta V / H}, \quad \widetilde{\boldsymbol{g}}=\frac{\boldsymbol{g}}{\|\boldsymbol{g}\|}
$$

and $S t=\rho g H^{2} / \eta V$ is the Stokes number characterizing the ratio between gravity and viscous forces. As stated before, Re is the Reynolds number which represents a ratio between inertial and viscous contributions. The dimensionless form of the mass conservation equation is $\widetilde{\nabla} \cdot \widetilde{\boldsymbol{u}}=0$.

In a typical slot coating flow of particle suspensions, a macroscopic analyses of the flow can be made in order to evaluate the contribution of each term in Eq. ( $\left[\begin{array}{ll}2.3 \\ )\end{array}\right)$. In this regard, considering a characteristic length and velocity proportional to the coating gap $(H \approx 100 \mu m)$ and substrate velocity $(V \approx 0.1 \mathrm{~m} / \mathrm{s})$, respectively; a suspension viscosity $\eta \approx 10^{-1} \mathrm{~Pa} \cdot \mathrm{s}$ and $\rho \approx 10^{3} \mathrm{~kg} / \mathrm{m}^{3}$, the Reynolds and Stokes numbers are $\mathcal{O}\left(10^{-1}\right)$ which are significantly low so that inertial and gravity effects can be neglected (Carvalho \& Kheshgi, 2000). Also, assuming a particle radius $a \approx 1 \mu \mathrm{m}$, the Knudsen number, $K n=a / H$, is $\mathcal{O}\left(10^{-2}\right)$ and low enough so that the continuum hypothesis is guaranteed (Batchelor, 1967). In addition to that, coating flows are laminar and, ideally, steady and two-dimensional (Carvalho, ए996). As a result, the flow in a typical slot coating application is governed by the Stokes equations

$$
\nabla \cdot \boldsymbol{u}=0
$$

and

$$
\nabla \cdot \boldsymbol{\sigma}=0
$$

Newton's second law of motion defines that the rate of change in linear momentum of a given body equals the sum of all external forces acting upon it. Among them, one can highlight body forces per units of mass and surface forces per units of volume. In Eq. ([2.6]) the term related to surface forces is the divergence of the stress tensor, $\nabla \cdot \boldsymbol{\sigma}$, and this term reveals the behavior of a fluid element which is in contact to the surrounding medium. The stress tensor $\boldsymbol{\sigma}$ is a second order tensor which can be understood as a sum of an isotropic or spherical part and a non-isotropic or deviatoric part (Batchelor, 1967). Moreover, an isotropic tensor is one whose components are invariant in relation to rotation. For instance, the identity tensor $\boldsymbol{I}$, by definition, is a second order isotropic tensor that serves as a basis for the calculation of other isotropic tensors of the same order (Aris, 1962). In this sense, the stress tensor can be expressed as 


$$
\boldsymbol{\sigma}=-p \boldsymbol{I}+\boldsymbol{\Sigma}^{d}
$$

in which $p=-1 / 3(\operatorname{tr} \boldsymbol{\sigma})$ is a mechanical pressure and $\boldsymbol{\Sigma}^{d}$ is the deviatoric or non-isotropic part of the stress tensor which is intrinsically related to the existence of motion in the fluid. From a phenomenological point of view, the departure from a spherical shape (i.e. equilibrium) of an element of fluid represents a transport of molecular momentum or internal friction that is accounted by the deviatoric stress tensor neglecting elastic effects. In this case, for ideal or stationary fluids, the stress tensor $\boldsymbol{\sigma}$ reduces to the spherical part of Eq. (区.7), i.e, $\boldsymbol{\sigma}=-p \boldsymbol{I}$.

For an incompressible and inelastic material, the deviatoric stress is a function of the rate-of-strain tensor given by

$$
\dot{\gamma}=\nabla \boldsymbol{u}+\nabla \boldsymbol{u}^{T}
$$

so that

$$
\Sigma^{d}=\Sigma^{d}(\dot{\gamma})
$$

As this work is about shear sensitive particle suspensions, we assume that the constitutive equation incorporates the concept of a shear-rate-dependent viscosity. Also, it should be sensitive to local particle concentration, $\phi$, so that $\eta=\eta(\phi, \dot{\gamma})$, where $\dot{\gamma}=|\dot{\gamma}| / \sqrt{2}$. More details about the viscosity model used in this work will be presented in the following subsection. With this in mind, and treating the suspension as a generalized Newtonian fluid, the deviatoric stress tensor can be modeled as

$$
\Sigma^{d}=\eta(\phi, \dot{\gamma}) \dot{\gamma}
$$

In Eq. (ए.Tा) $\dot{\gamma}=2 \boldsymbol{E}$ where $\boldsymbol{E}$ is the rate-of-deformation tensor that corresponds to the symmetric part o the velocity gradient tensor. In fact, a given second order tensor $\boldsymbol{A}$ may be decomposed as a sum of a symmetric part and an antisymmetric part, respectively, using the following identity: $\boldsymbol{A}=\frac{1}{2}\left(\boldsymbol{A}+\boldsymbol{A}^{T}\right)+\frac{1}{2}\left(\boldsymbol{A}-\boldsymbol{A}^{T}\right)$ (Aris, 1.962)

Thereupon, applying this result to Eqs. ([2.]) and ([2.2), one can obtain the most common form of the stress tensor and momentum equations of a generalized Newtonian fluid in a complete form as follows 


$$
\boldsymbol{\sigma}=-p \boldsymbol{I}+2 \eta(\dot{\gamma}, \phi) \boldsymbol{E}
$$

and

$$
\rho\left(\frac{\partial \boldsymbol{u}}{\partial t}+\boldsymbol{u} \cdot \nabla \boldsymbol{u}\right)=\nabla \cdot[-p \boldsymbol{I}+2 \eta(\dot{\gamma}, \phi) \boldsymbol{E}]+\rho \boldsymbol{g}
$$

\subsection{1}

\section{Viscosity Model}

In the flow of suspensions, three main forces coexist in different degrees according to physical parameters of the flow. Firstly, there are colloidal forces that act attracting or repealing particles interacting with other particles. In this sense, repulsion may occur due to the presence of surfactants coated on the surface of the particle or as a result of electrostatic charges. On the other hand, attraction might be related to van der Waals forces between particles or due to opposing charges on different particles. Secondly, for particle sizes $a \leq 1 \mu \mathrm{m}$ the ever-present Brownian random force is of significant importance (Barnes et al, 198.9). For particle sizes above this threshold, random thermal agitation of the fluid molecules is not strong enough to overcome inertial effects so that the particle Peclet, $P e_{a}$, number is very high. It is important to highlight that the Peclet number on the scale of the particle is determined by $P e_{a}=a^{2} \dot{\gamma}_{c} / \mathcal{D}$ and expresses a ratio between advective and Brownian diffusive contributions. In this latter relation, $\mathcal{D}=k_{B} T / 6 \pi \eta a$ is the Stokes-Einstein coefficient where $k_{B} \approx 1.380 \times 10^{-23} \mathrm{~J} \cdot \mathrm{K}^{-1}$ is the Boltzmann constant, $T \approx 300 \mathrm{~K}$ is the absolute temperature, $a$ is the particle radius and $\dot{\gamma}_{c}$ is a typical local shear rate of the flow. Finally, the last important force acting on the scale of the particles is the viscous force which is related to hydrodynamic or particle-fluid interactions.

In this work, we consider concentrated suspensions of spherical monodispersed colloidal particles with diameters $a \approx 1 \mu \mathrm{m}$ so that we are in the limit where Brownian restoring forces are still important. As a result, when these particles are introduced in a fluid at rest they tend to randomly assume a state of thermodynamic equilibrium also known as rest state (Larson, 1999); Macosko, 1994). In a common slot coating flow there are regions of low local shear rate, such as at the center of the feed slot region, and regions of high local shear rate near solid walls. Under those circumstances, at regions of low shear rates the particles tend move around each other in order to make the overall flow to happen and hence the viscosity is high. As the local Peclet number in those regions is very low, Brownian motion dominates the forces 
imposed by shear. In this sense, the distribution of particles remains almost the same and, consequently, the viscosity is approximately constant defining the so-called low shear-rate Newtonian plateau. On the other hand, at regions of slightly higher rates of shear, the random and cohesive particle structure tends to orientate in the direction of the flow in such a way that Brownian forces cannot restore the previous random state. In this case, the particles experience a lower resistance to move past each other and, therefore, the viscosity is also lower. In fact, in regions where the shear rate is even greater, this structure is so effectively oriented that it is possible to detect layers of particles bounded by layers of the continuous phase medium (Chen et al, 1.994). At this stage, the viscosity assumes its minimum value (i.e., the suspension shear-thins) and this well defined structure gives birth to the second well known Newtonian plateau at high rates of shear. Experiments have shown that at even higher shear rates the suspension may experience shear-thickening due to a shear-induced breakup of this layered structure (सoftman, 1972). Also, shear-thickening may appear due to a transition from a non-frictional rheology, where particles are well separated by lubrication layers, to another one dominated by frictional contacts (Seto et al, 2013; Wyart \& Cates, 2014). All of these phenomena put together show or predict the general viscosity curve of a typical shear sensitive suspension (Hinch, [201]; Laun, 1984).

In the light of what have been discussed so far, shear sensitive suspensions of hard spheres have a shear rate dependant viscosity which is also dependent on local particle concentration. In this case, we consider the viscosity model proposed by Cross (11970) defined as

$$
\eta(\dot{\gamma}, \phi)=\eta_{\infty}(\phi)+\frac{\eta_{0}(\phi)-\eta_{\infty}(\phi)}{1+b P e_{a}}
$$

where $b$ and $n$ are rheological fitting parameters and $\eta_{0}(\phi)$ and $\eta_{\infty}(\phi)$ refer to the asymptotic values of viscosity at very low and very high shear rates, respectively. Still in Eq. ([.T.3),$P e_{a}$ is the particle Peclet number calculated locally as $P e_{a}=\eta_{s} \dot{\gamma} a^{3} / k_{B} T$ where $\eta_{s}$ is the solvent viscosity. Note that the latter two viscosities depend only on local particle concentration and are modeled in accordance to the model presented by Krieger \& Dougherty (195.9) so that

$$
\eta_{0}(\phi)=\eta_{0}\left(1-\frac{\phi}{\phi_{m-0}}\right)^{-\alpha_{0}}
$$

and 


$$
\eta_{\infty}(\phi)=\eta_{0}\left(1-\frac{\phi}{\phi_{m-\infty}}\right)^{-\alpha_{\infty}} .
$$

In Eqs. (2.14) and (2.15) $\alpha_{0}$ and $\alpha_{\infty}$ are material constants and $\phi_{m-0}$ and $\phi_{m-\infty}$ are the maximum packing fractions or crowding factors for both low and high shear rate Newtonian plateaus. At particle concentrations above this limiting value, the particles start to jam and the viscosity diverges. The values of the rheological parameters in equations $([2.5),([2.14)$ and $([2.15)$ were fitted according to experimental observations made by Choi \& Krieger (1986). In this regard, we assume that $b=5.7, \phi_{m-0}=0.579, \phi_{m-\infty}=0.631, \alpha_{0}=1.85$ and $\alpha_{\infty}=1.67$. In addition to that, Cross' model is able to capture a well defined shear-thinning pattern between these two Newtonian plateaus as depicted in Fig. [2.]. It is important to note that this model directly incorporates the effect of particle size. If the particles are very small or very large such that the $P e_{a}<<10^{-2}$ or $P e_{a}>>10^{2}$, the viscosity is not a function of the local shear rate and only varies with the local concentration.

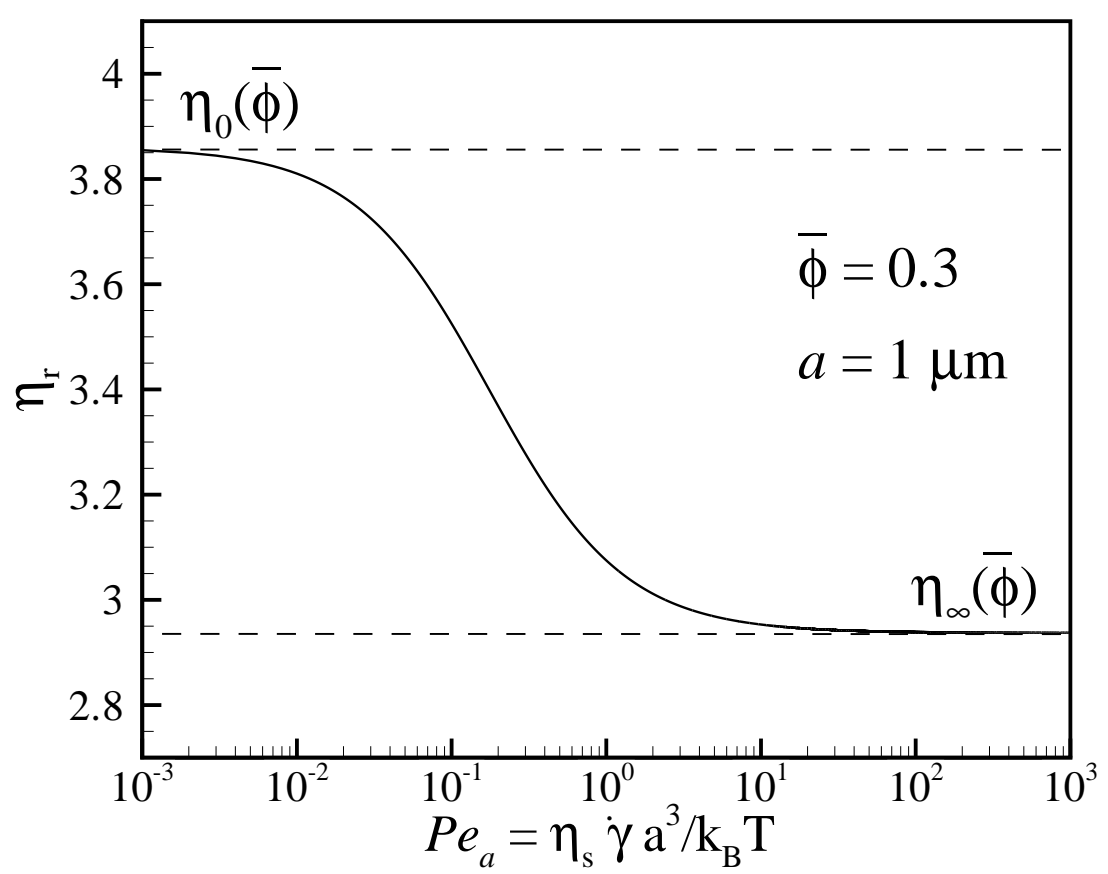

Figure 2.1: Cross' non-Newtonian viscosity model for a concentrated suspension at $\bar{\phi}=0.30$ and particle size $a=1 \mu \mathrm{m}$. Note that $\eta_{r}=\eta / \eta_{s}$ is a dimensionless viscosity. 


\subsection{2}

\section{Boundary Conditions: Momentum Equation}

Boundary conditions for the momentum equation can specify either velocity or traction, or a relationship between both. In the case of coating flows, the most common boundary conditions are sketched in Fig. 2.2 which are:

1. No-Slip or no-penetration: At solid/liquid interfaces the velocity of the liquid is the same as the velocity of the surface. In this sense,

$$
\boldsymbol{u}=\boldsymbol{u}_{w}
$$

where $\boldsymbol{u}_{w}$ is the velocity of the wall or the solid boundary.

2. Local slip or Navier slip: According to the works presented by Huh \& Scriven (IT7T) and Silliman ([T79), the movement over a solid/liquid/gas interface violates the adherence of the no-slip condition as this latter hypothesis leads to infinite high stresses (i.e. velocity gradients) in the vicinity of the dynamic contact line. In order to overcome this stress singularity, the no-slip condition can be replaced by the Navier slip condition so that the velocity discontinuity is proportional to the shear stress at the wall. With this in mind, this boundary condition can be written as follows

$$
\boldsymbol{t}_{w} \cdot\left(\boldsymbol{u}-\boldsymbol{u}_{w}\right)=\beta \boldsymbol{t}_{w} \cdot\left(\boldsymbol{n}_{w} \cdot \boldsymbol{\sigma}\right)
$$

where $\beta$ is the slip coefficient, $\boldsymbol{t}_{w}$ and $\boldsymbol{n}_{w}$ are the unit tangential and normal vectors, respectively. This relation is valid for low Reynolds numbers situations, typical of coating flows, and, note that, in Eq. ([2.]), if $\beta=0$ the no-slip condition is recovered. The slip coefficient is an empirical parameter since the flow near a dynamic contact line is still not fully understood.

3. Contact line/Contact angle: In order to apply this boundary condition, either the position of the contact line is specified or the inclination of the free surface at this region is prescribed by an apparent contact angle given by

$$
\boldsymbol{n}_{w} \cdot \boldsymbol{n}_{f s}=\cos \left(\theta_{\alpha}\right)
$$


in which $\boldsymbol{n}_{w}$ and $\boldsymbol{n}_{f s}$ are the unit outward normal vectors to the solid wall and free surface, respectively, and recall that this angle is applied at the contact line. Also, the magnitude of the contact angle should be determined experimentally and this parameter can be related to a dynamic or static contact line depending on the application.

4. Inflow and Outflow: In typical slot coating operations the region of study is bounded by inflow and outflow planes. The former, at the die feeding slot and, the latter, at the end of the final coated film located downstream. At the inflow plane, the suspension is assumed to have a uniform concentration profile, i.e. with a constant viscosity $\eta=\eta(\bar{\phi})$, and the flow is considered fully developed so that a parabolic velocity profile is specified:

$$
\boldsymbol{n} \cdot \boldsymbol{u}=-\frac{6 Q}{H}\left[\left(\frac{x}{H}\right)-\left(\frac{x}{H}\right)^{2}\right]
$$

where $\boldsymbol{n}$ is the unit vector normal to the boundary surface, $Q$ is the flow rate fed into the coating die which defines the thickness $t$ of the liquid layer deposited onto the substrate, i.e., $t=Q / V$. On the other hand, at the outflow plane, the flow is assumed to be fully developed and hence $\boldsymbol{n} \cdot \nabla \boldsymbol{u}=0$.

5. Force Balance at liquid/gas interfaces: Considering the gas is inviscid and free of inertial effects, the shear stress at the liquid/gas interface is zero and hence the normal liquid stress must be a balance by two contributions: the gas pressure and capillary pressure at the interface. Therefore,

$$
\boldsymbol{n} \cdot \boldsymbol{\sigma}=\frac{1}{C a} \frac{d \boldsymbol{t}}{d s}-\boldsymbol{n} P_{a m b}
$$

where $C a$ is the Capillary number, $s$ is a given distance over the free surface, $\boldsymbol{t}$ and $\boldsymbol{n}$ are the unit tangent and normal vectors to the free surface, respectively.

6. Kinetic Condition: This boundary condition imposes that there is no flow through a liquid/gas interface in such a way that the free surface can be treated as a streamline for a steady flow. In this region, 


$$
\boldsymbol{n} \cdot \boldsymbol{u}=0 \text {. }
$$

This condition implicitly defines the configuration of the free boundaries of the flow together with relation (2.201).

1) $\underline{\text { No-Slip }}$

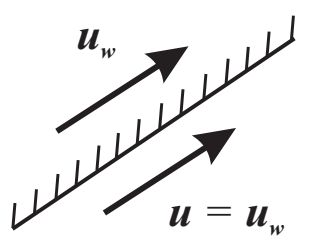

4) Inflow and outflow

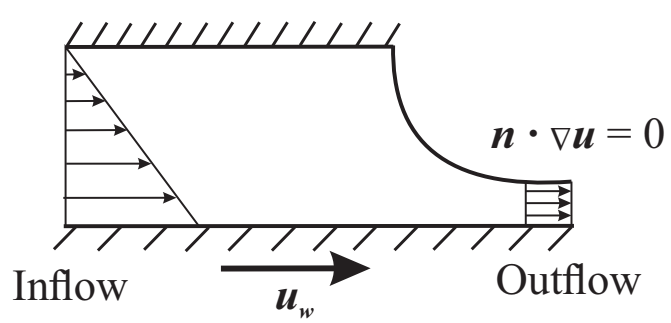

2/3) Dynamic contact line/angle

Free surface

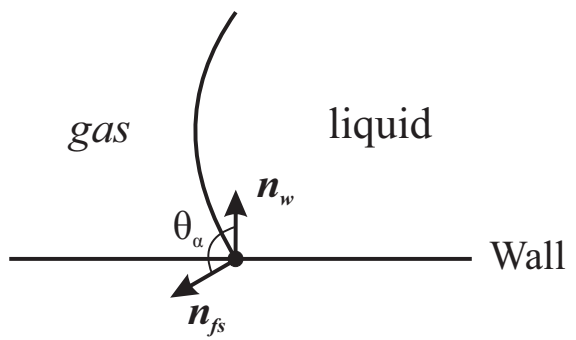

5) Capillary Pressure

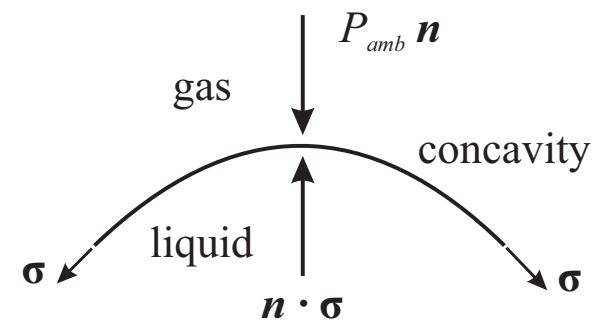

6) $\underline{\text { Kinematic }}$

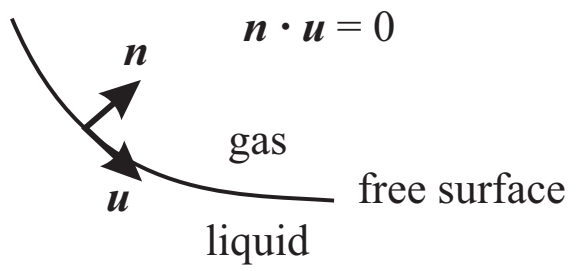

Figure 2.2: Boundary conditions for the conservation of momentum equations.

\section{2}

\section{Particle Migration Equation}

In the field of continuum mechanics, many laws state that the total amount of a certain quantity in a medium is either invariant or varies according to external 
influences such as molecular transport through a control volume surface. In this manner, consider an arbitrary volume of fluid, $v$, bounded by a surface $S$ and that $d \boldsymbol{S}$ is the area vector normal to a differential area $d S$. Now, consider an arbitrary extensive quantity of the fluid per unit of volume given by $\theta(\boldsymbol{x}, t)$. Also, let $\boldsymbol{N}_{\theta}$ be the total diffusive flux of this quantity, $\boldsymbol{u} \theta$ the transport flux of $\theta$ and $\zeta$ the effective density of source strength. In this manner, the conservation law for this extensive quantity is as follows (Batchelor, 1967),

$$
\frac{\partial \theta}{\partial t}+\nabla \cdot\left(\boldsymbol{N}_{\theta}+\boldsymbol{u} \theta\right)=\zeta
$$

In the case that $\theta$ is equal to the particle volume concentration $\phi$ and assuming that there is no generation or loss of particles in the domain, Eq. ([2.22) reduces to

$$
\frac{\partial \phi}{\partial t}+\nabla \cdot\left(\boldsymbol{N}_{\phi}+\boldsymbol{u} \phi\right)=0
$$

which is the typical equation that describes the transport of particles in a general flow.

In order to better understand the net migration of particles, it is important to briefly explain how they interact with each other. In cases of dilute regimes, due to the linearity of the equations of motion in the scale of the particles, kinematic reversible interactions might occur which means that the particles tend to recover the same trajectory if the flow is reversed. However, for concentrated suspensions, irreversible interactions may happen in situations where three-body collisions come into consideration leading to a shear-induced self-diffusion (Eckestein et al, 1.977; Cunha \&. Hinch, 1.996). Leighton \& Acrivos (1987a) have shown experimentally that the difference between theoretical and experimental predictions for the latter diffusion is approximately of one orderof-magnitude. In this regard, they proposed that this additional flux is due to irreversible particle-particle interactions that drives the particles from regions of high to low local concentrations, against gradients in concentration, and from regions of high to low collision frequencies, which scales with $\dot{\gamma} \phi$, against gradients in local shear rate.

With regards to particle migration modelling, we follow the work presented by Leighton \& Acrivos (1987a, [1) and Phillips et al. (1992) in which the total flux of particles $\boldsymbol{N}_{\phi}$ in Eq. ([2.23) is solely accounted for irreversible particle-particle interactions. This global flux can be understood as a contribu- 
tion of different factors such as: Brownian diffusion, effect of spatially varying interaction frequency, effect of spatially varying viscosity, migration due to the curvature of the streamlines, concentration-dependent empirical parameters and also sedimentation. In the present work we use the hypothesis of neutrally buoyant particles, so that sedimentation is not important. In this sense, particle flux is modeled by an extension of the Diffusive Flux Model (DFM) and more details with respect to different migration mechanisms are presented in the following subsections.

\subsection{1}

\section{Effect of spatially varying interaction frequency}

A general shear flow can be understood as a combination of shearing surfaces sliding in relative motion to each other. Then, embedded in these shearing surfaces are particles that interact with one another in such a way that irreversible two-body collisions dominates. In fact, as this particle-particle interactions are irreversible, the particles tend to migrate from a region of higher frequency of collisions to regions of lower frequency of collisions in the direction normal to their plane of shear (Fig. [2.3). According to the works of Leighton \& Acrivos (1.987a, b) and Phillips et al. (1.992), in a concentrated suspension the collision frequency experienced by a test particle scales with $\dot{\gamma} \phi$ in a typical shear flow. Moreover, the change in those collisions over a distance $\mathcal{O}(a)$ is proportional to $a \nabla(\dot{\gamma} \phi)$ and it is assumed that the particle migration velocity is linearly proportional to this variation in collision frequency. Also, considering that an irreversible interaction generates a displacement $\mathcal{O}(a)$, the flux of particles due to spatially varying interaction collision frequency is given by

$$
\boldsymbol{N}_{c}=-k_{c} a^{2} \phi \nabla(\dot{\gamma} \phi)
$$

where $k_{c}$ is a diffusive constant $\mathcal{O}(1)$ to be determined experimentally. Equation ([2.24), can be rewritten as follows

$$
\mathbf{N}_{c}=-k_{c} a^{2}\left(\phi^{2} \nabla \dot{\gamma}+\phi \dot{\gamma} \nabla \phi\right)
$$

so that the two terms on the right-hand side give rise to two opposing mechanisms discussed previously. To illustrate, assume a concentrated suspension with uniform particle concentration. Now, if a varying shear rate is imposed on 
this suspension, a flux of particles will happen due the term $\sim \nabla \dot{\gamma}$ from high to low shear rate regions. At the same time, the particle concentration profile will change, giving birth to a second opposing flux from regions of higher concentrations to lower concentrations due to the term $\sim \nabla \phi$.

(a)

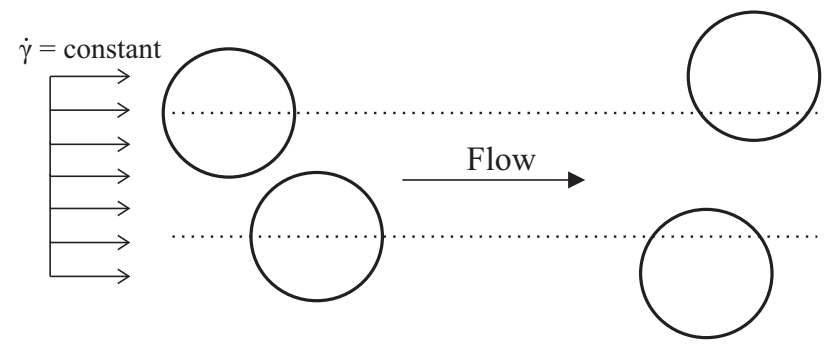

(b)
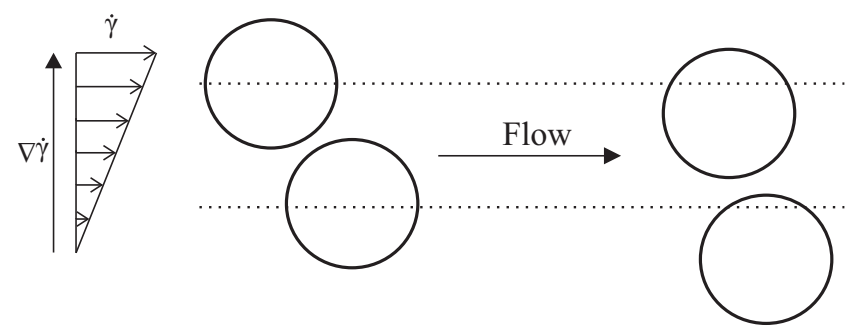

Figure 2.3: Irreversible two-body collisions: (a) constant shear rate and (b) spatially varying shear rate

\subsection{2}

\section{Effect of spatially varying viscosity}

Still under the light of the works developed by Leighton \& Acrivos ([प987a, $\underline{\mathrm{b}}$ ) and Phillips et al. (1992), another important migration flux is highlighted, which is related to spatially varying viscosity. By the way, as we are dealing with shear sensitive suspensions, i.e. $\eta=\eta(\dot{\gamma}, \phi)$, this additional flux takes into account gradients in concentration and also shear rate. Furthermore, a gradient in viscosity can be understood as a resistance to the motion experienced by a test particle undergoing a two-body irreversible collision. Consequently, it tends to migrate towards regions in which the resistance to motion is lower so that this flux of particles is shaped from regions of high to low viscosity as sketched in Fig. [2.4.

In order to better understand this phenomenon, a scaling analysis is available in which, during each irreversible interaction, the magnitude of the displacement $\mathcal{O}(a)$ experienced by a given test particle scales with $\left(a^{2} / \eta\right) \nabla \eta$. In this manner, since the rate of interactions is $\dot{\gamma} \phi$, the drift velocity of each test particle is proportional to $\dot{\gamma} \phi\left(a^{2} / \eta\right) \nabla \eta$ which leads to an expression for the overall effective diffusion when multiplied by $\phi$, so that, 


$$
\boldsymbol{N}_{\eta}=-k_{\eta} \dot{\gamma} \phi^{2} \frac{a^{2}}{\eta} \nabla \eta
$$

where $k_{\eta}$ is a diffusive constant of $\mathcal{O}(1)$ to be determined by experiments. Again, Eq. (2.26) can be rearranged as follows

$$
\boldsymbol{N}_{\eta}=-k_{\eta} \dot{\gamma} \phi^{2} \frac{a^{2}}{\eta}\left(\frac{d \eta}{d \phi} \nabla \phi+\frac{d \eta}{d \dot{\gamma}} \nabla \dot{\gamma}\right)
$$

owing to the fact the viscosity is a function of both local particle concentration and shear rate.

(a)
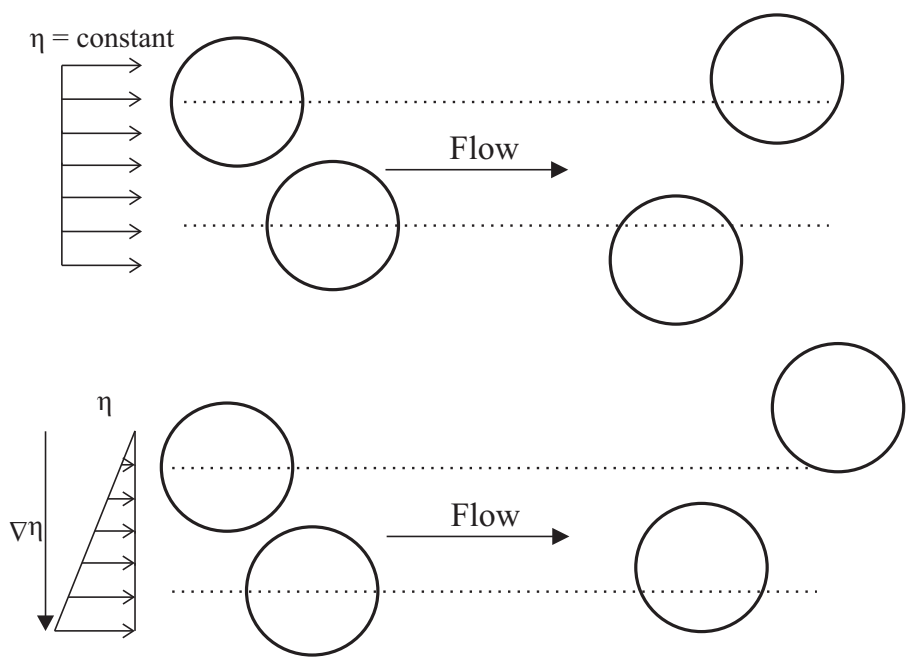

Figure 2.4: Irreversible two-body collision: (a) constant viscosity and (b) spatially varying viscosity.

\subsection{3}

\section{Curvature-induced migration}

Chow et al. (1994) employed NMR ${ }^{\boxplus}$ imaging to study particle migration in Couette and parallel plate viscometers. In Couette flow, the authors confirmed the observations made by Leighton \& Acrivos (1987a, b) that the particles tend to migrate from regions of high to low shear rates due to shear-induced migration. However, in torsional flow (i.e., parallel plates viscometer) they verified no change in the particle concentration profile in the radial direction. In the latter geometry, the shear rate monotonically increases from the center to the outer part of the parallel plate device so that one should expect to see an

\footnotetext{
${ }^{1}$ Nuclear Magnetic Ressonance
} 
inward particle migration. In an effort to explain this behavior, Krishnan et al. (11995) suggested that there should be another migration mechanism in the outward direction to account for this experimental observation and the authors defined this extra diffusion as a curvature-induced migration. Additionally, the curvature decreases from the inner to the outer part of the parallel plate viscometer which induces particle migration in the same direction. As a result, this curvature-induced migration is then expected to balance with the inward shear-induced migration and is given by

$$
\boldsymbol{N}_{\kappa}=k_{\kappa} \kappa a^{2} \dot{\gamma} \phi^{2} \boldsymbol{n}_{\boldsymbol{S T}}
$$

in which $k_{\kappa}$ is a empirical parameter, $\kappa$ is the local curvature of the streamline and $\boldsymbol{n}_{\boldsymbol{S T}}$ is the unit radially outward vector normal to the streamline. In Eq. $(2 \cdot 28)$ it is assumed that the particles migrate in the same direction of the unit vector normal to a streamline with curvature $\kappa$ in a frame-invariant formulation as depicted in Fig. 2.5. The expressions for $\boldsymbol{n}_{\boldsymbol{S T}}$ and $\kappa$ in a steady-state regime are as follows

$$
\boldsymbol{n}_{\boldsymbol{S T}}=\frac{[(\boldsymbol{u} \cdot \nabla \boldsymbol{u}) \cdot \nabla \boldsymbol{u}] \boldsymbol{u}-|\boldsymbol{u}|^{2} \boldsymbol{u} \cdot \nabla \boldsymbol{u}}{|\boldsymbol{u}||(\boldsymbol{u} \cdot \nabla \boldsymbol{u}) \times \boldsymbol{u}|}
$$

and

$$
\kappa=\frac{|(\boldsymbol{u} \cdot \nabla \boldsymbol{u}) \times \boldsymbol{u}|}{|\boldsymbol{u}|^{3}} .
$$

In this work we assume that $k_{\kappa}$ is equal to $k_{c}$ since these two mechanisms should be in balance with each other (Krishnan et al, 1995; Kim et al, 20108).

\subsection{4}

\section{Volume fraction dependency of phenomenological parameters}

The volume fraction dependency of the phenomenological parameters $k_{c}$ and $k_{\eta}$ was first presented in experimental and theoretical investigations made by Tetlow et al ([1998) and Graham et aL (1998). The latter authors proposed that particle roughness might not be the principal source of flux so that an alternative cause of irreversibility shall occur. With this in mind and considering that the original DFM proposed by Phillips et al. (1.992) takes into account irreversible two-body collisions, they verified that the empirical diffusive parameters should be concentration-dependent. In this dissertation, 


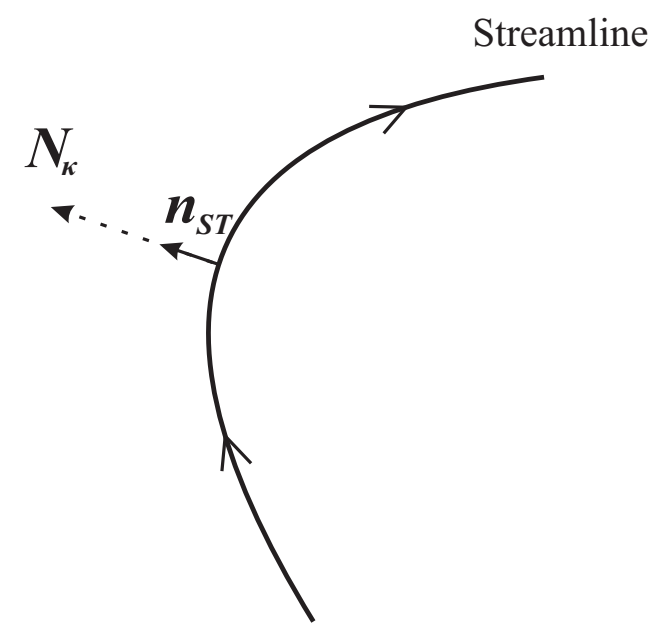

Figure 2.5: Direction of the migration due to the curvature of the streamline.

we present an analysis of this approach and follow the predictions shown by Kim et al. (2008) which developed a set of volume fraction dependent parameters given by: $k_{c}=1.5 \phi k_{c}^{P}, k_{\eta}=k_{\eta}^{P}$ and $k_{\kappa}=0.75 k_{c}^{P}$, where $k_{c}^{P}=0.41$ and $k_{\eta}^{P}=0.62$ are the empirical parameters determined by the seminal work of Phillips et al. (1992).

\subsection{5}

\section{Brownian Diffusion}

The botanist Robert Brown, in 1827, was the first to observe that colloidal particles suspended in a viscous fluid are subjected to an isotropic random movement caused by constant collisions of the molecules of the fluid upon them also known as Brownian movement in his honor (Brown, 1827). In a pioneering work, Einstein (1.906) apud Gontijo (2013) investigated this phenomena and determined a analytical relation for the mean squared displacement of a test particle undergoing Brownian motion as

$$
<x^{2}>=2 \mathcal{D} t
$$

in which $\left\langle>\right.$ denotes an average over a set of numbers, $x^{2}$ represents the squared displacement, $\mathcal{D}$ is a Brownian diffusion coefficient and $t$ is the time over which the observation was held. In general, anisotropic diffusion coefficients can be expressed as a second order tensor $\mathcal{D}$, however, due to the isotropic nature of the Brownian movement, this tensorial quantity is reshaped as $\mathcal{D}=\mathcal{D} \boldsymbol{I}$. In addition to that, the magnitude of $\mathcal{D}$ is proportional to the molecular agitation of the fluid (i.e., temperature) and to the level o mobility of a test particle so that 


$$
\mathcal{D}=k_{B} T \mathcal{M}
$$

where $\mathcal{M}$ is a mobility tensor of a solid particle given by

$$
\mathcal{M}=\frac{\boldsymbol{I}}{6 \pi \eta a}
$$

Note that the diffusion coefficient represented by Eqs. ([2.32) and ([2.33) is the well-known Stokes-Einstein diffusion coefficient so that $\mathcal{D}=k_{B} T / 6 \pi \eta a$. Under those circumstances, Brownian diffusion of particles can be shaped as

$$
\mathbf{N}_{b}=-\mathcal{D} \nabla \phi
$$

and, in case of colloidal suspensions, Brownian forces tend to restore the microstructure to a previously random state, as explained before in this Chapter.

In order to evaluate the importance of this type of diffusion, a comparison with the others mechanisms of migration must be made. Also, to clarify the following analysis the transport equation of particles will the presented in its dimensionless form. In this sense, rearranging Eq. ([2.2.3]), the Diffusive Flux Model reduces to

$$
\frac{\partial \phi}{\partial t}+\boldsymbol{u} \cdot \nabla \phi+\phi \nabla \cdot \boldsymbol{u}=-\nabla \cdot \boldsymbol{N}_{\phi}
$$

where for a stationary regime of an incompressible fluid, $\nabla \cdot \boldsymbol{u}=0$ as $D \rho / D t=0$, so that,

$$
\boldsymbol{u} \cdot \nabla \phi+\nabla \cdot \boldsymbol{N}_{\phi}=0 .
$$

From a simple scaling analysis of all migration mechanisms studied so far, one shall demonstrate that $\boldsymbol{N}_{\phi}$ have a scale of velocity. With this in mind, consider the following characteristic relations for the terms in Eq. (2.36])

$$
\widetilde{\nabla}=H \nabla, \quad \widetilde{\boldsymbol{u}}=\frac{\boldsymbol{u}}{V}, \quad \widetilde{\boldsymbol{N}}_{\phi}=\frac{\boldsymbol{N}_{\phi}}{a \dot{\gamma}_{c}}
$$

where $a \dot{\gamma}_{c}$ is a typical velocity on the scale of the particles and $\dot{\gamma}_{c}=V / H$ is a 
characteristic shear rate of a typical slot coating flow. In this case, recall that $a$ is the particle size, $V$ is the substrate velocity and $H$ is the gap between the feed slot and the moving substrate. After a few algebraic steps, one shall obtain the dimensionless form of Eq. (2.36) as follows

$$
\widetilde{\boldsymbol{u}} \cdot \widetilde{\nabla} \phi+\frac{H}{a} \frac{1}{P e} \widetilde{\nabla} \cdot \widetilde{\boldsymbol{N}}_{\phi}=0
$$

in which $P e=(V H) / a^{2} \dot{\gamma}_{c}$ is the Peclet number that denotes a balance between advection and diffusive contributions. In an alternative form, since $\dot{\gamma}_{c}=V / H$, the Peclet number can be rewritten as $P e=(H / a)^{2}$ so that Eq. (2.38) reduces to

$$
\widetilde{\boldsymbol{u}} \cdot \widetilde{\nabla} \phi+\frac{a}{H} \widetilde{\nabla} \cdot \widetilde{\boldsymbol{N}}_{\phi}=0
$$

Note from Eq. (2.39) that for a fixed coating gap, the overall diffusion of particles depends on their size. In order words, if the particles are too small, shear- and curvature- induced particle migration which scale with $a^{2}$ shall be very weak and, eventually, Brownian diffusion may dominate since $\mathcal{D} \sim 1 / a$. On the other hand, for greater particle sizes, such as $a \approx 1 \mu \mathrm{m}$, those mechanisms may be of the same order of magnitude. In order to investigate their contribution separately, recall that the total flux of particles may be evaluated in terms of $\boldsymbol{N}_{\phi}=\boldsymbol{N}_{c}+\boldsymbol{N}_{\eta}+\boldsymbol{N}_{b}$ where $\boldsymbol{N}_{c}, \boldsymbol{N}_{\eta}, \boldsymbol{N}_{b}$ and $\boldsymbol{N}_{\kappa}$ are given by Eqs. ([2.25), ([2.27), (2.34) and ([2.28). As a result, the Diffusive Flux Model, in its dimensional form, can be rewritten as

$\boldsymbol{u} \cdot \nabla \phi+\nabla \cdot\left[-k_{c} a^{2}\left(\phi^{2} \nabla \dot{\gamma}+\phi \dot{\gamma} \nabla \phi\right)-k_{\eta} \dot{\gamma} \phi^{2} \frac{a^{2}}{\eta}\left(\frac{d \eta}{d \phi} \nabla \phi+\frac{d \eta}{d \dot{\gamma}} \nabla \dot{\gamma}\right)-\mathcal{D} \nabla \phi+k_{\kappa} \kappa a^{2} \dot{\gamma} \phi^{2} \boldsymbol{n}_{\boldsymbol{S T}}\right]=0$

and, rearranging the terms in Eq. ( 2.40$)$ it is possible to show that

$$
\boldsymbol{u} \cdot \nabla \phi+\nabla \cdot\left(\mathcal{D}_{\phi} \nabla \phi+\mathcal{D}_{\dot{\gamma}} \nabla \dot{\gamma}-\mathcal{D} \nabla \phi+\mathcal{D}_{\kappa} \boldsymbol{n}_{\boldsymbol{S T}}\right)=0
$$

where $\mathcal{D}_{\phi}=-k_{c} a^{2} \phi \dot{\gamma}-k_{\eta} \dot{\gamma} \phi^{2} \frac{a^{2}}{\eta} \frac{d \eta}{d \phi}, \mathcal{D}_{\dot{\gamma}}=-k_{c} a^{2} \phi^{2}-k_{\eta} \dot{\gamma} \phi^{2} \frac{a^{2}}{\eta} \frac{d \eta}{d \dot{\gamma}}, \mathcal{D}$ is the Stokes-Einstein diffusion coefficient and $\bar{D}_{\kappa}=k_{\kappa} \kappa a^{2} \dot{\gamma} \phi^{2}$ is a curvatureinduced coefficient. Now, considering a colloidal suspension with particle size $a=1 \mu \mathrm{m}$ and a ratio of $k_{c} / k_{\eta}=0.66$, one can observe that $\overline{\mathcal{D}}_{\phi}, \overline{\mathcal{D}}_{\dot{\gamma}}$ and $\overline{\mathcal{D}}_{\kappa}$ are $\mathcal{O}\left(10^{-8}\right)$ and $\mathcal{D}$ is $\mathcal{O}\left(10^{-17}\right)$ so that Brownian diffusion can be neglected. Note that Brownian diffusion is not considered because its contribution to particle migration is at least one order of magnitude lower than the other migration 
mechanisms. However, Brownian forces are still present in the scale of the particles and are important in order to restore a random rest state of the microstructure which is reflected by a pseudo-plastic behavior of the fluid for a given range of local shear rate and particle size.

\subsection{6}

\section{Boundary Conditions: Diffusive Particle Transport}

The boundary conditions imposed for the particle migration equation in a typical slot coating process are:

1. No-flux of particle: It is assumed that there is no-flux of particles at solid/liquid or liquid/gas interfaces so that,

$$
\boldsymbol{n} \cdot\left(\phi \boldsymbol{u}+\boldsymbol{N}_{\phi}\right)=0 .
$$

2. Inflow and Outflow: At the inflow region, a prescribed concentration profile is usually imposed. In this work, a uniform concentration profile is set at the feed slot entrance. At the outflow region, the boundary condition of no-diffusive flux of particles given by Eq. (2.42) is applied:

$$
\boldsymbol{n} \cdot \boldsymbol{N}_{\phi}=0 .
$$




\section{Numerical Methodology}

In general, the great majority of physical problems can be modeled by differential equations. In this sense, numerical approaches are usually employed in order to solve them by discretizing the domain under investigation into a set of algebraic equations valid in each node or element of the mesh. For instance, the Finite Difference Method (FDM) is a classical model to solve differential equations that is simple to implement and accurate mostly in cases of regular geometries. In parallel, in order to tackle real engineering problems with complex boundaries, a class of variational methods evolved during the $19^{\text {th }}$ and $20^{\text {th }}$ century due to investigations performed by renowned researches like Rayleigh, Ritz and Galerkin. Among these methods the Galerkin/Finite Element Method (GFEM) stems out as an important and efficient option (Donea \& Huerta, 2003).

The Galerkin/Finite Element Method is based on a formulation of weighted residuals in which the exact solution of a given differential equation, posed in a infinite space, is projected orthogonally on a finite subspace. This projection is achieved by a scalar product between the residual of the differential equation and a given weighting function which, in an integral form, gives rise to the weak form of the formulation. Therefore, the exact solution is approximated by a linear combination of piecewise basis functions that spans this subspace. As a consequence, in principle, the larger the subspace, the more accurate is the solution. In the Galerkin method, the weighting functions used in the orthogonal projection are equal to the basis functions that spam the subspace of approximate solution (Gresho \& Sani, 11998).

As shown in Fig. B.d, a general slot coating flow is as a free-surface problem since the flow domain has two liquid-gas interfaces, viz. downstream and upstream of the feed slot die. For this reason, the physical domain where the conservation equations (i.e. mass, momentum and particle transport) are posed is also unknown and hence the coordinates of the free surfaces are likewise part of the solution. In this sense, the original set of partial differential equations is augmented by an additional couple of diffusive equations used in 
the mapping from an unknown domain to a geometrically simple reference and known domain. Details related to the solution method of a general free-surface problem are presented in the following sections.

\section{1}

\section{Formulation of Free Surface Problems}

In order to tackle a typical free-boundary problem efficiently, Christodolou \& Scriven (149.92); de Almeida (11995) presented an approach in which the set of all governing differential equations in the unknown or physical domain, $\Omega$, should be transformed into a set delineated in an equivalent geometrically simple and known reference domain, $\Omega_{0}$, as sketched in Fig. B.]. This procedure is achieved by the mapping $\boldsymbol{x}=\boldsymbol{x}(\boldsymbol{\xi})$ that connects both regions. Here, $\boldsymbol{x}$ and $\boldsymbol{\xi}$ are the position vectors that parameterizes both physical and reference domains, respectively, and the inverse mapping is given by $\boldsymbol{\xi}=\boldsymbol{\xi}(\boldsymbol{x})$.

The mapping is arbitrary and a common approach is to adopt quadrangular domains composed by unit squares as the reference domain. There are situations in which the physical domain is highly complex so that the reference region shall be subdivided into sub-domains connected to each other such as a slot coating flow depicted in Fig. B.]. As matter of fact, this mapping must obey two important constraints: $(i)$ the boundaries of the reference domain have to be mapped over the boundaries of the physical domain; and ( $i i)$ the mapping must be invertible, which means that

$$
\operatorname{det}\left(\nabla_{\boldsymbol{\xi}} \boldsymbol{x}\right) \neq 0
$$

for all $\boldsymbol{\xi}$ belonging to $\Omega_{0}$. In Eq. 3. , the term $\nabla_{\boldsymbol{\xi}} \boldsymbol{x}$ is the Jacobian matrix $\boldsymbol{J}$ of the mapping which, in two-dimensional problems, is given by

$$
\nabla_{\boldsymbol{\xi}} \boldsymbol{x} \equiv \boldsymbol{J}=\left(\begin{array}{cc}
\frac{\partial x}{\partial \xi} & \frac{\partial y}{\partial \xi} \\
\frac{\partial x}{\partial \eta} & \frac{\partial y}{\partial \eta}
\end{array}\right)
$$

where $\operatorname{det}(\boldsymbol{J})=|\boldsymbol{J}|$ is the Jacobian of the transformation.

In order to write the set of differential equations posed in the physical domain in terms of the coordinates of the reference domain, the spatial derivatives shall be written as follows 

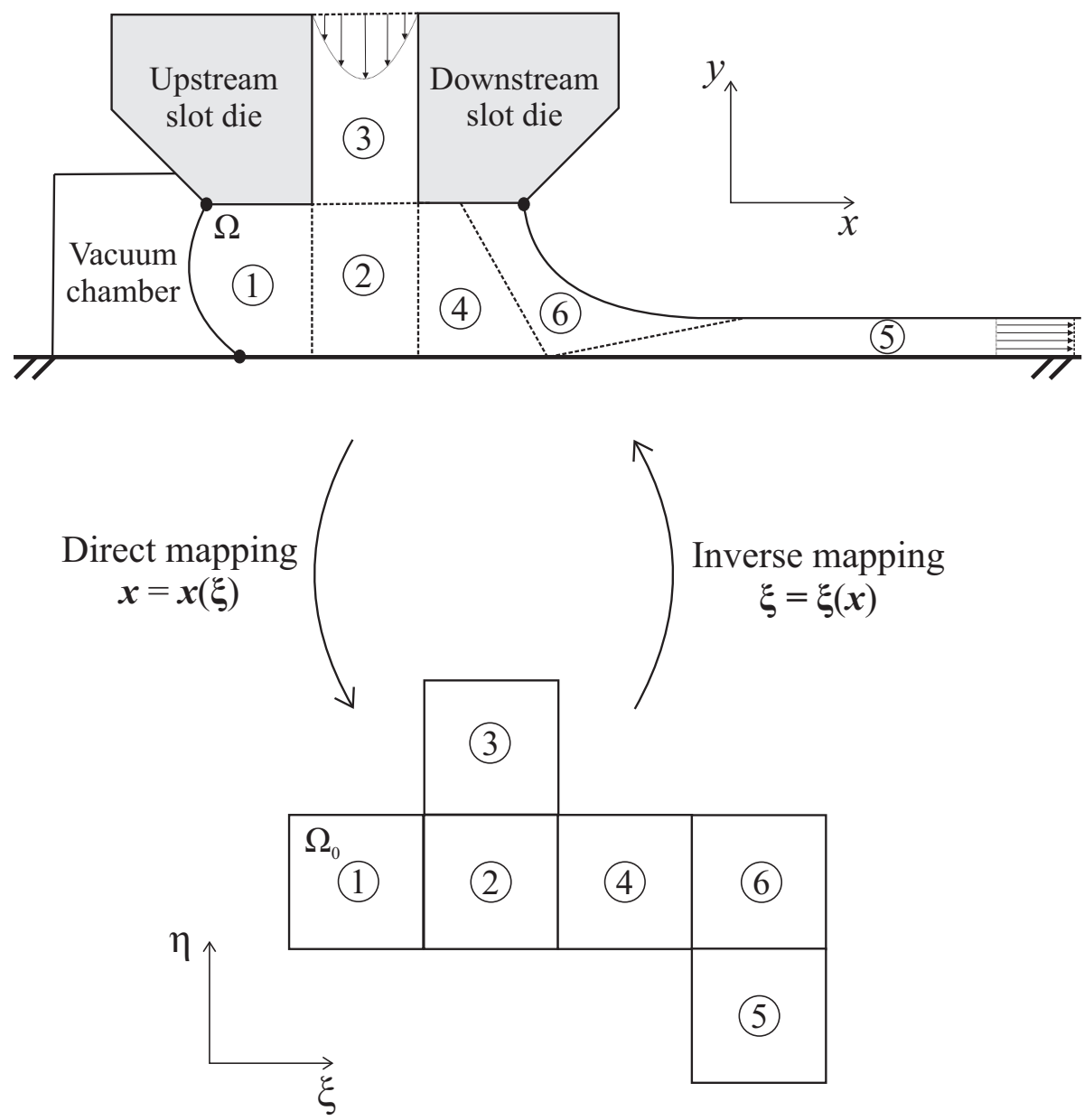

Figure 3.1: Mapping from the unknown physical domain to a given reference domain. The physical domain is transformed into regions geometrically simple composed by unit quadrangular domains (Adapted from Siqueira (2016)).

$$
\left(\begin{array}{c}
\frac{\partial \alpha}{\partial x} \\
\frac{\partial \alpha}{\partial y}
\end{array}\right)=\boldsymbol{J}^{-1}\left(\begin{array}{c}
\frac{\partial \alpha}{\partial \xi} \\
\frac{\partial \alpha}{\partial \eta}
\end{array}\right)
$$

where $\alpha=\alpha(\xi, \eta)$ is an arbitrary quantity. Moreover, area integrals over the physical domain are written in terms of the reference domain as $d \Omega=|\boldsymbol{J}| d \Omega_{0}$.

Again, the differential equations that define velocity, pressure and particle concentration fields together with the equations that govern the mapping are solved in the reference domain using the Galerkin/Finite Element method which will be discussed in detail in the present chapter. The mesh generation scheme will be presented next. 


\section{2}

\section{Mesh Generation Scheme}

In order to solve a free-surface problem, a mesh generation scheme is the crux of the matter. With this in mind, boundary conforming meshes can be generated either from an algebraic or from an elliptical mesh generation approach. The former one locates the mesh points by using a simple interpolation scheme and, despite its computational efficiency, this method requires a high interaction with the user and fails in cases of highly distorted meshes. On the other hand, the latter scheme relates mesh points of the physical domain to points of a reference domain and it provides inherent smoothness of the mesh while requiring less interaction of the user (Christodolou \& Scriven, 1992). This method was also studied and refined by Santos (11.94) and later on by Benjamin (11994) and is usually known as elliptic mesh generation. The main goal of this approach is to optimize the quality of the mesh by minimizing a functional which depends on the smoothness, orthogonality and density of the mesh. As a result, the inverse mapping that minimizes this functional is governed by the following elliptic differential equations

$$
\nabla \cdot\left(D_{\xi} \nabla \xi\right)=0
$$

and

$$
\nabla \cdot\left(D_{\eta} \nabla \eta\right)=0
$$

where $D_{\xi}$ and $D_{\eta}$ are diffusion like coefficients of the coordinates potentials that control the spacing between the curves of constant $\xi$ and $\eta$. These curves tessellate the reference domain into finite elements. The diffusion-like coefficients are obtained by interpolating prescribed stretching functions $f(\xi)$ and $g(\eta)$ along the boundaries, using the following relations:

$$
F(\xi, \eta)=\left(1-\frac{\eta}{\eta_{\max }}\right) f_{1}(\xi)+\frac{\eta}{\eta_{\max }} f_{2}(\xi)
$$

and

$$
G(\xi, \eta)=\left(1-\frac{\xi}{\xi_{\max }}\right) g_{1}(\eta)+\frac{\xi}{\xi_{\max }} g_{2}(\eta)
$$


so that $D_{\xi} \equiv \partial F / \partial \xi$ and $D_{\eta} \equiv \partial G / \partial \eta$. In an effort to write $\boldsymbol{x}=\boldsymbol{x}(\boldsymbol{\xi})$, Eqs. (B.4) and (B.5) must be transformed into the reference domain configuration. Fig. 3.2 illustrates the stretching functions used in the calculation of the diffusion-like coefficients of the mesh-generation scheme.

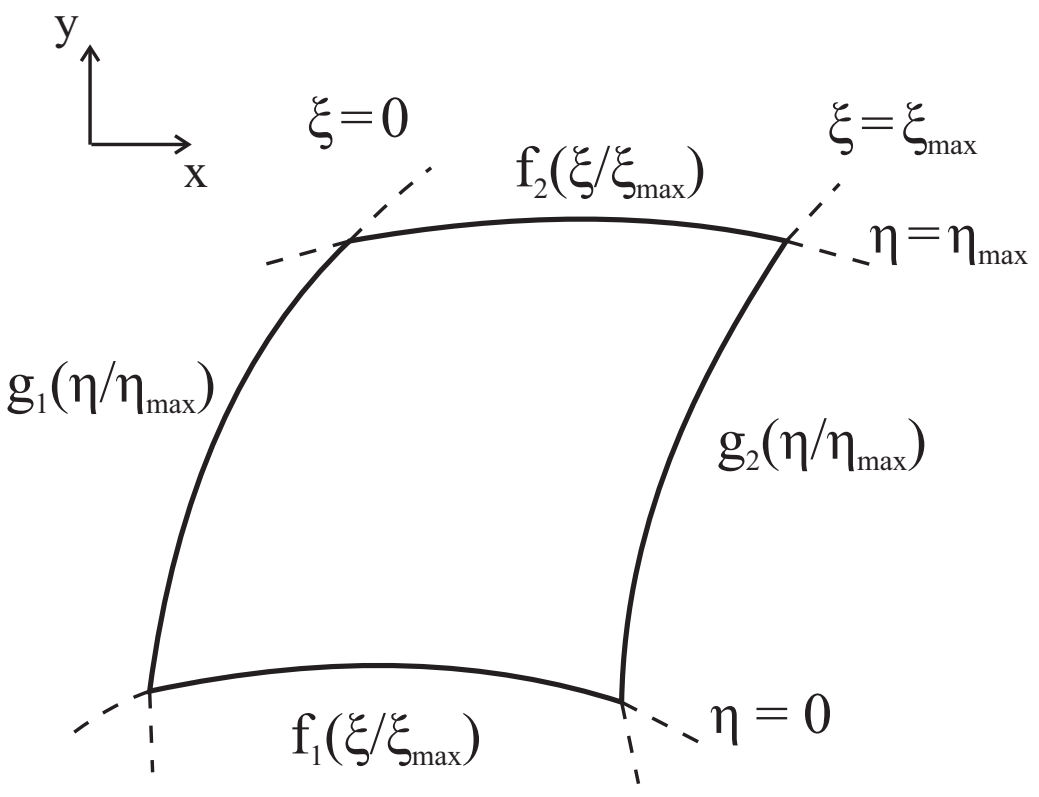

Figure 3.2: Stretching functions used in the calculation of the diffusion coefficients $D_{\xi}$ and $D_{\eta}$.

\subsection{1}

\section{Boundary Conditions: Mesh-Generation Equations}

In order to solve the set of partial differential Eqs. (3.4) and (B.5), boundary conditions must be employed. These conditions are shown in Fig. 3.3 and defined as follows.

1. Prescribed Geometry: this boundary condition is applied to all fixed boundaries except for free surfaces. In this sense, an equation that defines the shape of the boundary as a relation between the coordinates $x$ and $y$ is given as

$$
y=G(x),
$$

where $G$ is a prescribed function that describe the shape of fixed boundaries. 
2. Physical Condition: the shape of a free surface is generally determined by the physics of the problem. For instance, a kinematic condition implies that the liquid cannot cross the free surface, leading to $\boldsymbol{n} \cdot \boldsymbol{u}=0$. However, extra conditions are required in order to locate the mesh nodes and hence the position of the isolines of $\xi$ and $\eta$ over a specific interface. In this manner, there are three available options:

i Node distribution along boundary: in this case, stretching functions $f$ and $g$ are imposed in order to distribute the nodes over the boundary and control their spacing, so that

$$
\xi=f^{-1}(s)
$$

or

$$
\eta=g^{-1}(s)
$$

where $s$ is the arch-length coordinate along the boundary. More details about the shape of these stretching functions can be found in the work of Benjamin (11994).

ii Prescribed mesh angle at a boundary: the prescribed angle $\Phi$ between the isolines of $\xi$ and $\eta$ coordinates at the boundary, as shown in Fig.(3.3), is determined by

$$
\boldsymbol{n} \cdot \nabla \xi=|\nabla \xi| \cos (\Phi)
$$

in which $\boldsymbol{n}$ is the unit normal vector pointing outward the physical domain. Note that, if the scalar product presented in Eq. (B.]) is zero, then a orthogonal condition is achieved.

iii Prescribed mesh angle at a corner: this condition is satisfied by imposing an angle $\beta$ between the outward unit vectors, $\boldsymbol{n}_{1}$ and $\boldsymbol{n}_{2}$, to both converging lines that meet at the corner as shown in Fig. 3.3. As a result,

$$
\boldsymbol{n}_{1} \cdot \boldsymbol{n}_{2}=\cos (\beta)
$$

where $\beta=\left(180^{0}-\alpha\right)$. 
1) Prescribed Geometry

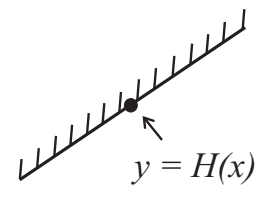

i) Node Distribution

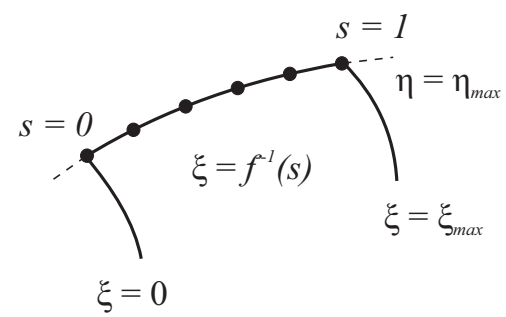

iii) Corner Angle

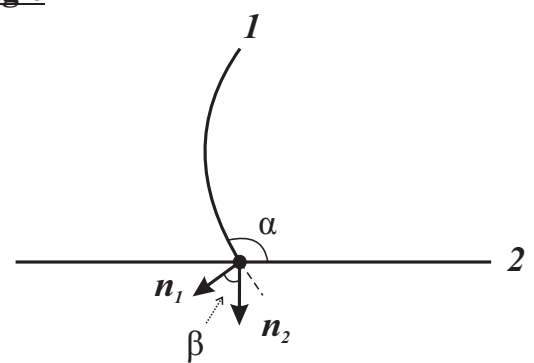

Figure 3.3: Boundary conditions for the equations (B.4) and (B.5) that denotes the mapping between the reference and physical domains.

\section{3}

\section{Interpolated Velocity Gradient}

The motivation for the use of an interpolated velocity gradient relies on the formulation of a general GFEM itself. Since the velocity field is approximated by a linear combination of Lagrangian biquadratic basis functions defined in each domain, along their boundaries, the velocity is continuous but the derivatives are not. In order to deal with the singularity of the weak formulation that has terms proportional to the integral of the second derivative of the velocity, Szad et al. ([1995) proposed that an interpolated velocity gradient should be used in place of the general velocity gradient as an independent field in the form

$$
\boldsymbol{G}=\nabla \boldsymbol{u}
$$


in an effort to improve the stability and convergence of the numerical method. However, in incompressible fluid flows the computed velocity gradient is not exactly divergence-free (i.e. $\operatorname{tr}(\boldsymbol{G})=\nabla \cdot \boldsymbol{u}=0$ ) in every point. The divergence of the velocity is zero only in a weak sense. With this in mind, Pasquali (2000) and Pasquali \& Scriven (2002) proposed a new formulation for the interpolated velocity gradient as follows

$$
\boldsymbol{G}=\nabla \boldsymbol{u}-\frac{(\nabla \cdot \boldsymbol{u}) \boldsymbol{I}}{\operatorname{tr} \boldsymbol{I}}
$$

where $\boldsymbol{I}$ is the identity tensor. This relation guarantees that $\operatorname{tr}(\boldsymbol{G})=0$ in every point of the domain.

This last contribution closes the set of differential equations that govern the phenomenon under study in this research, composed by the equations of incompressible mass ([2.5); momentum ([2.6); particle transport (2.36); elliptic mesh generation (B.4) and (B.5); and, finally, interpolated velocity gradient (3.14). By means of organization, these equations are rewritten as follows:

- Mass Balance:

$$
\nabla \cdot \boldsymbol{u}=0
$$

- Momentum Balance:

$$
\nabla \cdot \boldsymbol{\sigma}=0
$$

where $\boldsymbol{\sigma}$ is given by Eq. ([.]);

- Particle Transport:

$$
\boldsymbol{u} \cdot \nabla \phi+\nabla \cdot \boldsymbol{N}_{\phi}=0
$$

- Elliptic Mesh Generation:

$$
\nabla \cdot\left(D_{\xi} \nabla \xi\right)=0
$$


and

$$
\nabla \cdot\left(D_{\eta} \nabla \eta\right)=0
$$

- Interpolated Velocity Gradient:

$$
\boldsymbol{G}-\nabla \boldsymbol{u}+\frac{(\nabla \cdot \boldsymbol{u}) \boldsymbol{I}}{\operatorname{tr} \boldsymbol{I}}=0
$$

This set of differential equations is solved by Galerkin's method with finite element basis functions. In order to do so, these equations are written in an integral weak form, as addressed in the subsequent section.

\section{4}

\section{Weighted Residual Form}

It is important to remember that to employ the weighted residual method to solve a system of partial differential equations by Galerkin's approach, the exact solution is projected on a finite subset spanned by a finite array of basis functions. As a result, this method requires the residue of this approximation to be, at least in average, equal to zero along the whole domain under investigation. In this sense, the generic weighted residual form or weak form of the problem addressed in this work is given by

$$
\begin{aligned}
R_{c} & =\int_{\Omega}(\nabla \cdot \boldsymbol{u}) w_{c} d \Omega=0 \\
R_{m} & =\int_{\Omega}(\nabla \cdot \boldsymbol{\sigma}) \cdot \boldsymbol{w}_{m} d \Omega=0 \\
R_{\phi} & =\int_{\Omega}\left(\boldsymbol{u} \cdot \nabla \phi+\nabla \cdot \boldsymbol{N}_{\phi}\right) w_{\phi} d \Omega=0 \\
R_{\xi} & =\int_{\Omega}\left[\nabla \cdot\left(D_{\xi} \nabla \xi\right)\right] w_{\xi} d \Omega=0 \\
R_{\eta} & =\int_{\Omega}\left[\nabla \cdot\left(D_{\eta} \nabla \eta\right)\right] w_{\eta} d \Omega=0 \\
R_{\boldsymbol{G}} & =\int_{\Omega}\left[\boldsymbol{G}-\nabla \boldsymbol{u}+\frac{(\nabla \cdot \boldsymbol{u}) \boldsymbol{I}}{\operatorname{tr} \boldsymbol{I}}\right]: \boldsymbol{w}_{\boldsymbol{G}} d \Omega=0,
\end{aligned}
$$

where $\boldsymbol{w}$ and $w$ are tensor and scalar weighting functions, respectively. In fact, $w$ can be expressed as a linear combination basis functions so that, 


$$
w(x)=\sum_{i=1}^{N} w_{i} \psi_{i},
$$

where the basis functions $\psi_{i}$ span the subspace of dimension $N$ that approximates the projection space.

Now, consider the following tensorial identities

$$
\boldsymbol{\sigma}: \nabla \boldsymbol{w}_{m}=\nabla \cdot\left(\boldsymbol{\sigma} \cdot \boldsymbol{w}_{m}\right)-(\nabla \cdot \boldsymbol{\sigma}) \cdot \boldsymbol{w}_{m}
$$

and

$$
\nabla \cdot(\psi \boldsymbol{a})=\boldsymbol{a} \cdot \nabla \psi+\psi \nabla \cdot \boldsymbol{a}
$$

where $\psi$ and $\boldsymbol{a}$ are arbitrary scalars and vectors, respectively. Using these relations and applying the Gauss-Green theorem to Eqs. (3.16), (3.18) and (उ.19) it is possible to show that

$$
\begin{aligned}
R_{m}^{i} & =\int_{\Omega} \boldsymbol{\sigma}: \boldsymbol{w}_{m} d \Omega-\int_{\Gamma}(\boldsymbol{n} \cdot \boldsymbol{\sigma}) \cdot \boldsymbol{w}_{m} d \Gamma=0, \\
R_{\xi}^{i} & =-\int_{\Omega} D_{\xi} \nabla \xi \cdot \nabla w_{\xi} d \Omega+\int_{\Gamma} \boldsymbol{n} \cdot\left(D_{\xi} \nabla \xi\right) w_{\xi}=0, \\
R_{\eta}^{i} & =-\int_{\Omega} D_{\eta} \nabla \eta \cdot \nabla w_{\eta} d \Omega+\int_{\Gamma} \boldsymbol{n} \cdot\left(D_{\eta} \nabla \eta\right) w_{\eta}=0,
\end{aligned}
$$

in which $\Gamma$ is the boundary of the physical domain $\Omega, \boldsymbol{n}$ is the outward unit vector to the boundary and $i=1,2, \ldots, N$.

A special attention is required in order to write Eq. (3.17) in the same way because the term $\nabla \cdot \boldsymbol{N}_{\phi}$ presents explicit second derivatives in velocity in the term $\nabla \dot{\gamma}$. Since the velocity field is a linear combination of piecewise polynomials, second derivatives are singular and must be avoided. In this sense, $\dot{\gamma}$ is written in terms of the second invariant of the rate-of-strain tensor, $\dot{\gamma}$, which is now a function of the interpolated velocity gradient, so that,

$$
\dot{\gamma}=\boldsymbol{G}+\boldsymbol{G}^{T}
$$

Under the light of the weighted residuals method and using Eq. ([2.4]) without the Brownian diffusive term, it follows that 


$$
R_{\phi}^{i}=\int_{\Omega}(\boldsymbol{u} \cdot \nabla \phi) w_{\phi} d \Omega+\int_{\Omega} \nabla \cdot\left(\overline{\mathcal{D}}_{\phi} \nabla \phi+\overline{\mathcal{D}}_{\dot{\gamma}} \nabla \dot{\gamma}+\overline{\mathcal{D}}_{\kappa} \boldsymbol{n}_{\boldsymbol{S T}}\right) w_{\phi} d \Omega=0
$$

Using the identity $(\sqrt{323})$ on equation $(\overline{328})$ and applying the GaussGreen theorem, the residual for the particle transport equation assumes the form

$$
\begin{aligned}
R_{\phi}^{i} & =\int_{\Omega}\left[(\boldsymbol{u} \cdot \nabla \phi) w_{\phi}-\overline{\mathcal{D}}_{\phi} \nabla \phi \cdot \nabla w_{\phi}-\overline{\mathcal{D}}_{\dot{\gamma}} \nabla \dot{\gamma} \cdot \nabla w_{\phi}-\overline{\mathcal{D}}_{\kappa} \boldsymbol{n}_{\boldsymbol{S T}} \cdot \nabla w_{\phi}\right] d \Omega \\
& -\int_{\Gamma} \boldsymbol{n} \cdot\left(\overline{\mathcal{D}}_{\phi} \nabla \phi+\overline{\mathcal{D}}_{\dot{\gamma}} \nabla \dot{\gamma}+\overline{\mathcal{D}}_{\kappa} \boldsymbol{n}_{\boldsymbol{S T}}\right) w_{\phi} d \Gamma=0 .
\end{aligned}
$$

where, again, $\boldsymbol{n}_{\boldsymbol{S T}}$ is the local unit normal vector to a given streamline.

\subsection{1}

\section{Expansion of the Unknown Fields}

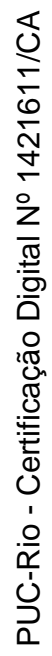

$$
\boldsymbol{G}=\left[\begin{array}{cc}
\sum_{j=1}^{M} G_{u x}^{j} \psi_{j} & \sum_{j=1}^{M} G_{u y}^{j} \psi_{j} \\
\sum_{j=1}^{M} G_{v x}^{j} \psi_{j} & \sum_{j=1}^{M} G_{v y}^{j} \psi_{j}
\end{array}\right] .
$$


where $P_{j}, U_{j}, V_{j}, X_{j}, Y_{j}, \phi_{j}$ and $G_{u, v ; x, y}^{j}$ are the coefficients used in the linear combination which are unknown a priori. It is important to note that the weighting functions used in the mass balance residue are the same as those used in the interpolation of the approximate pressure field and hence the pressure is adjusted in order to conserve the mass in each element.

In addition to that, the main feature of these basis functions is that they are non-zero only over a small part of the domain shedding light to the concept of an element. As a result, using the elliptic mesh generation, by which the governing equations are set in a reference domain, an interplay between elemental and global perspectives is established. From a numerical point of view, the choice of the right combination of basis functions leads to stable formulation and, in this research, they obey the Babuska-Brezzi condition summarized in Table B.4.] (Babuska, 1973; Franca \& Frey, 1991). In this table, the indexes $n$ and $m$ refer to the number of basis functions that must be used in each linear combination in each element. In this work, quadrilateral elements are adopted to discretize the domain and details relative to this type of element are presented next.

Table 3.1: Table with different combination of basis functions $\varphi$ and $\psi / \chi$

\begin{tabular}{|l|l|l|}
\hline Elements & $\varphi$ & $\psi$ or $\chi$ \\
\hline \hline Quadrilateral Elements & $\checkmark$ bilinear $(n=4)$ & $\checkmark$ constant $(m=1)$ \\
& $\checkmark$ biquadratic $(n=9)$ & $\checkmark$ bilinear $(m=4)$ \\
& $\checkmark$ biquadratic $(n=9)$ & $\checkmark$ linear discontinuous $(m=3)$ \\
& $\checkmark$ bicubic $(n=16)$ & $\checkmark$ biquadratic $(m=9)$ \\
\hline Triangular Elements & $\checkmark$ biquadratic $(n=6)$ & $\checkmark$ linear $(m=6)$ \\
& $\checkmark$ cubic $(n=9)$ & $\checkmark$ quadratic $(m=6)$ \\
\hline
\end{tabular}

\section{4 .2}

\section{Element Type}

The philosophy of the finite element method is to focus on a small patch of the domain, also known as element, in which the basis functions are nonzero. The global point of view of a given physical problem is represented by a global matrix that is made up of an interposition of local or element matrices. Moreover, the connection between the element nodes and global nodes is performed by a matrix called connectivity matrix.

In this research, quadrilateral elements are used which are classified as Lagrangian biquadratic basis functions, $\varphi$, for velocity, concentration and mesh; linear discontinuous basis functions, $\chi$, for pressure and bilinear basis functions, $\psi$, for the interpolated velocity gradient as shown in Fig. B.4. 


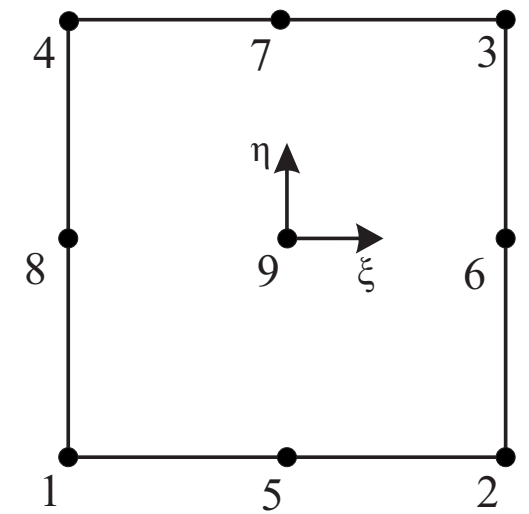

Figure 3.4: Node representation of a typical quadrangular element where $\xi$ and $\eta$ are local coordinates.

Each element is composed by nine nodes which leads to 64 local degrees of freedom. The correspondent degree of freedom related to each unknown variable is summarized in Table [3.4.2. .

Table 3.2: Relationship between local numbering and unknown coefficients degrees of freedom in each element.

\begin{tabular}{|c|c|}
\hline Local degree of freedom & Degree of freedom \\
\hline $1 \cdots 9$ & $Y_{1} \cdots Y_{9}$ \\
\hline $10 \cdots 18$ & $X_{1} \cdots X_{9}$ \\
\hline $19 \ldots 27$ & $V_{1} \cdots V_{9}$ \\
\hline $28 \cdots 36$ & $U_{1} \cdots U_{9}$ \\
\hline $37 \cdots 45$ & $\phi_{1} \cdots \phi_{9}$ \\
\hline $46 \cdots 48$ & $P_{1} \cdots P_{3}$ \\
\hline $49 \cdots 52$ & $G_{u x}^{1} \cdots G_{u x}^{4}$ \\
\hline $53 \cdots 56$ & $G_{u y}^{1} \cdots G_{u y}^{4}$ \\
\hline $57 \cdots 60$ & $G_{v x}^{1} \cdots G_{v x}^{4}$ \\
\hline $61 \cdots 64$ & $G_{v y}^{1} \cdots G_{v y}^{4}$ \\
\hline
\end{tabular}

\section{5}

\section{Solution Method}

The integrals of the residual formulation are solved in the reference domain, $\Omega_{0}$, using the Gaussian Quadrature Method with three points of integration in each direction. Thereupon, those integrals are written in the following form

$$
\int_{\Omega} F(x, y) d \Omega=\int_{\Omega_{0}} \widetilde{F}(\xi, \eta)|\boldsymbol{J}| d \Omega_{0}
$$


again, $|\boldsymbol{J}|$ is the Jacobian of the mapping. Also, the weighted residual method together with a linear interpolation of the unknown fields give rise to a system of non-linear algebraic equations which is solved with Newton's method with a numerical Jacobian. This system can be treated as $\boldsymbol{R}(\boldsymbol{c})=0$, where $\boldsymbol{c}=\boldsymbol{c}(\boldsymbol{x}, \boldsymbol{u}, \phi, \boldsymbol{G})$ is the global solution vector and $\boldsymbol{R}$ is a global residual vector. Newton's method linearizes the system of non-linear algebraic equations and consists in the solution of this linear system by means of the following iterative procedure:

$$
\begin{aligned}
& \boldsymbol{c}=\boldsymbol{c}_{0} \\
& \text { while }\|\boldsymbol{R}(\boldsymbol{c})\|>\epsilon \text { do } \\
& \quad \boldsymbol{J} \Delta \boldsymbol{c}=-\boldsymbol{R} \\
& \quad \boldsymbol{c}=\boldsymbol{c}+\Delta \boldsymbol{c}
\end{aligned}
$$

\section{end while}

Here, $\epsilon$ is the numerical tolerance of the method, $\boldsymbol{c}_{0}$ is an initial guess and $\boldsymbol{J}$ is the Jacobian matrix that denotes the sensibility of each residual equation in relation to each unknown variable of the problem, in other words,

$$
J_{i j}=\frac{\partial R_{i}}{\partial c_{j}}
$$

In Eq. ([3.36) $), i, j=1, \cdots, N_{D o f}$ and $N_{D o f}$ is the total number of basis functions coefficients (i.e. degrees of freedom) and residual equations. In this work the Jacobian matrix is calculated numerically through a central difference scheme so that

$$
J_{i j}=\frac{R_{i}\left(c_{1}, \cdots, c_{j}+\varepsilon, \cdots, c_{N_{D o f}}\right)-R_{i}\left(c_{1}, \cdots, c_{j}-\varepsilon, \cdots, c_{N_{D o f}}\right)}{2 \varepsilon}
$$

where $\varepsilon$ is a slight perturbation around an entry $j$ of the solution vector and this parameter is $\mathcal{O}\left(10^{-6}\right)$.

A few important remarks about the method are:

- At each iteration of Newton's method, the linear system $\boldsymbol{J} \Delta \boldsymbol{c}=-\boldsymbol{R}$ must be solved. Since the basis functions used in a GFEM are non-zero only in a few regions of the domain, the Jacobian matrix is sparce. In this work, each iteration step is performed using a robust LU frontal solver coupled with a pseudo-arc-length continuation in order to start with a satisfactory initial guess of the solution. Details about this procedure can be found in the following works de Almeida (1995); de Almeida et al. (1999) and Carvalho (1996); 
- If the problem is linear, the method converges in one single iteration;

- The convergence of the method is quadratic as the solution is approached.

\section{6}

\section{Numerical Aspects}

From Eqs. (2.2.5) and (2.27) it is possible to show that the Diffusive Flux Model is singular in regions where $\dot{\gamma}$ approaches zero such as the centerline of a tube or channel flow. On one hand, the term $\sim k_{c} a^{2} \phi^{2} \nabla \dot{\gamma}$ forces the particles to migrate to regions of lower shear rate (or zero shear rate if any); on the other hand, this flux is opposed by a contrary migration proportional to $\dot{\gamma} \phi$ as can be detected in the terms $\sim k_{c} \phi \dot{\gamma} \nabla \phi, \sim k_{\eta} \dot{\gamma} \phi^{2} a^{2} \eta^{-1}(\partial \eta / \partial \phi \nabla \phi)$ and $\sim k_{\eta} \dot{\gamma} \phi^{2} a^{2} \eta^{-1}(\partial \eta / \partial \dot{\gamma} \nabla \dot{\gamma})$. As a result, in regions where $\dot{\gamma} \rightarrow 0$, this opposing flux vanishes and the model predicts particle concentrations close to or equal to the maximum packing fraction which leads to a local infinite viscosity. Consequently, this theoretical inconsistency originates numerical convergence problems.

In order to overcome this singularity, in this work we employ a modified version of the non-local stress contribution to the shear rate as presented by Miller \& Morris (2006) and later on adapted by Ahmed \& Singh (2011) in which

$$
\dot{\gamma}_{\delta}=\delta \frac{U_{\max }}{H}
$$

where $\delta \approx 10^{-3}$ is a numerical parameter, $U_{\max }$ is the maximum suspension velocity and $H$ is a characteristic length (e.g., in a tube flow $H$ would be equal to the tube radius). In this sense, the non-local stress contribution is important in regions where $\dot{\gamma} \rightarrow 0$ so that $\dot{\gamma}=\dot{\gamma}_{\delta}$. This procedure avoids the divergence of the viscosity model and improves the numerical results by leading to more numerically stable problem. 


\section{Results and discussion}

The results of this work concern to the analysis of a slot coating process in which the coating liquid is a shear sensitive particle suspension. In this manner, a shear rate dependant viscosity model is employed which is also sensitive to local particle concentration. The main goal is to analyze the effect of operating conditions on the particle distribution in the deposited coated layer. In this work, particle migration mechanisms were computed in accordance with the three models: Model I, original DFM proposed by Phillips et al. (1992); Model II, which is a modified Phillips model with curvature-induced migration; and, lastly, Model III which is a modified Model II with concentration-dependant phenomenological diffusive constants following the investigations presented by Kim et al. (2018) . The predictions of the model that consider particle migration are compared to the solution of the flow problem assuming that the particle concentration is uniform on the flow.

\section{1}

\section{Numerical Validation}

In an effort to validate the numerical code used in this dissertation, we compared the numerical results of velocity and particle concentration profiles with the correspondent analytical solutions presented by Phillips et al. (1992) for a fully-developed tube flow of a non-colloidal suspension of spherical mono-modal and rigid particles. The latter authors used a constant ratio of $k_{c} / k_{\eta}=0.66$ to calculate the exact solutions for velocity and particle concentration. The analytical particle concentration profile is given by

$$
\phi=\frac{\phi_{m}}{1+\beta \tilde{r}}
$$

where $\beta=\left(\phi_{m}-\phi_{w}\right) / \phi_{w}$ is a numerical parameter, $\phi_{w}$ is the concentration at the wall and $\tilde{r}=r / R$ is a dimensionless radial coordinate where $R$ is the tube radius. Still in Eq. (4.]), $\phi_{m}=0.68$ is the maximum packing fraction used in Krieger's model. In this validation the particle size adopted was $a=10 \mu \mathrm{m}$ (i.e., 
non-colloidal suspension) so that the viscosity model is a function of particle concentration only

$$
\eta(\phi)=\eta_{s}\left(1-\frac{\phi}{\phi_{m}}\right)^{-1.82}
$$

It is remarkable that the analytical velocity profile is calculated as a function of the exact concentration profile since the viscosity model varies with local particle concentration. In order to clarify this assumption, consider the momentum conservation equation in cylindrical coordinates as follows

$$
\frac{1}{r} \frac{d}{d r}\left(r \tau_{r z}\right)=\frac{\partial P}{\partial z}
$$

where $\tau_{r z}=\dot{\gamma} \eta(\phi)$ in which $\dot{\gamma}=d u / d r$. Integrating Eq. (4.3) once and using the relation for $\tau_{r z}$, it can be shown that

with the boundary condition of no-slip velocity at the tube wall, $u(R)=0$. With this in mind, let $G=-\partial P / \partial z$ where $G$ is constant for each bulk concentration in such a way that

$$
\frac{d u}{d r}=-\frac{1}{2} G \frac{r}{\eta}
$$

Moreover, note that $\tilde{\eta}=\eta / \eta_{s}$ is a dimensionless viscosity and hence Eq. (4.5) can be written as

$$
\frac{d}{d r}\left(\frac{u}{R G / \eta_{s}}\right)=-\frac{\tilde{r}}{2 \tilde{\eta}}
$$

where $\tilde{u}=u /\left(R G / \eta_{s}\right)$ is also a dimensionless velocity. Recall that $\tilde{\eta}$ is a function of particle concentration which also depends on the radial position. Hence, a dimensionless velocity profile can be calculated by integrating the following equation 


$$
\frac{d \tilde{u}}{d \tilde{r}}=-\frac{\tilde{r}}{2 \widetilde{\eta}}
$$

considering the corresponding boundary condition $\tilde{u}(1)=0$. Consequently, if the exact concentration profile given by Eq. (4.]) is used in Eq. (4.7), then it is possible to determine the analytical velocity profile for a given analytical particle distribution.

The numerical results were developed by applying the GFEM where the whole set of residual forms related to the problem (i.e., mass, momentum, particle migration and interpolated velocity gradient) were solved in cylindrical coordinates in a two-dimensional flow through a tube. Also, a mesh with $25 \times 30$ quadrangular elements was employed in the numerical simulations. The flow domain is shown in Fig. 4.0 and the boundary conditions used for momentum and particle migration equations were:

1. Inlet: Prescribed parabolic velocity profile of a Newtonian fluid with viscosity $\eta=\eta(\bar{\phi})$ and constant particle concentration profile $\phi(r)=\bar{\phi}$;

2. Outlet: Fixed pressure and fully-developed velocity and concentration profiles so that $\boldsymbol{n} \cdot \nabla \boldsymbol{u}=0$ and $\boldsymbol{n} \cdot \nabla \phi=0$;

3. At the wall: no-slip boundary condition for velocity and no-penetration or flux of particles $\left(\boldsymbol{n} \cdot \boldsymbol{N}_{\phi}\right)=0$;

4. Centerline of the tube: symmetry for the velocity profile and no flux of particles.

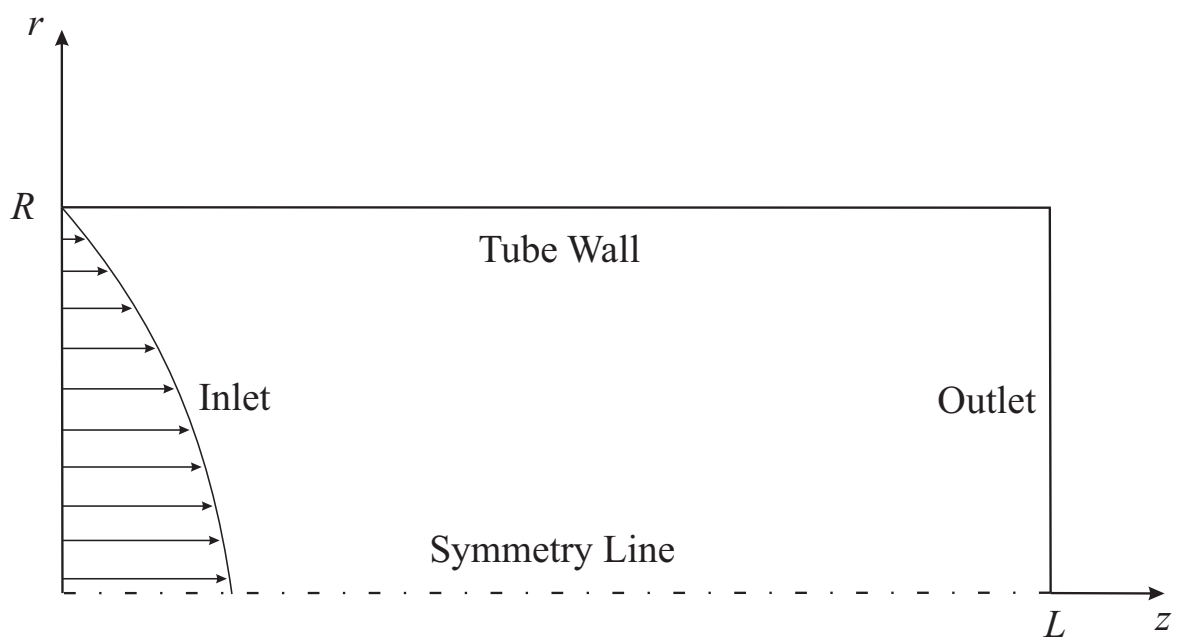

Figure 4.1: Flow domain of a cylindrical tube used in the numerical validation. 
The parameters used as input data for the simulations were: particle radius $a=10 \mu \mathrm{m}$, tube radius $R=1.0 \mathrm{~mm}$, tube length $L=10.0 \mathrm{~mm}$ and bulk concentrations $\bar{\phi}=0.3,0.4,0.5$ and 0.6. Figures 4.2 and 4.3 show the results for both velocity and particle concentration profiles for a set of bulk concentrations $\bar{\phi}$. As can be seen, the results agree very well with the exact solutions for velocity and particle concentration profiles given by Eqs. (4.]) and (4.7), respectively. Also, the imposed flow rate was adjusted such that the pressure difference was the same for all cases.

Note that for all bulk concentrations, particle concentration increases from the wall towards the centerline of the tube. As a result, the velocity profile becomes more blunted in relation to the parabolic Newtonian fluid one presenting lower centerline velocity. In other words, the flow rate decreases as the bulk concentration increases. For instance, for $\bar{\phi}=0.6$ the centerline velocity is $20 \%$ of the maximum velocity of a plain Newtonian fluid.

It is also important to highlight that the numerical predictions for the particle concentration profiles present small deviations from the analytical solution, specially, near the centerline of the tube. Recall that, in this region, $\dot{\gamma}=\dot{\gamma}_{\delta}$ and the local particle concentration never reaches the maximum packing fraction, $\phi_{m}=0.68$, as predicted by the original Diffusive Flux Model proposed by Phillips et al. (1992).

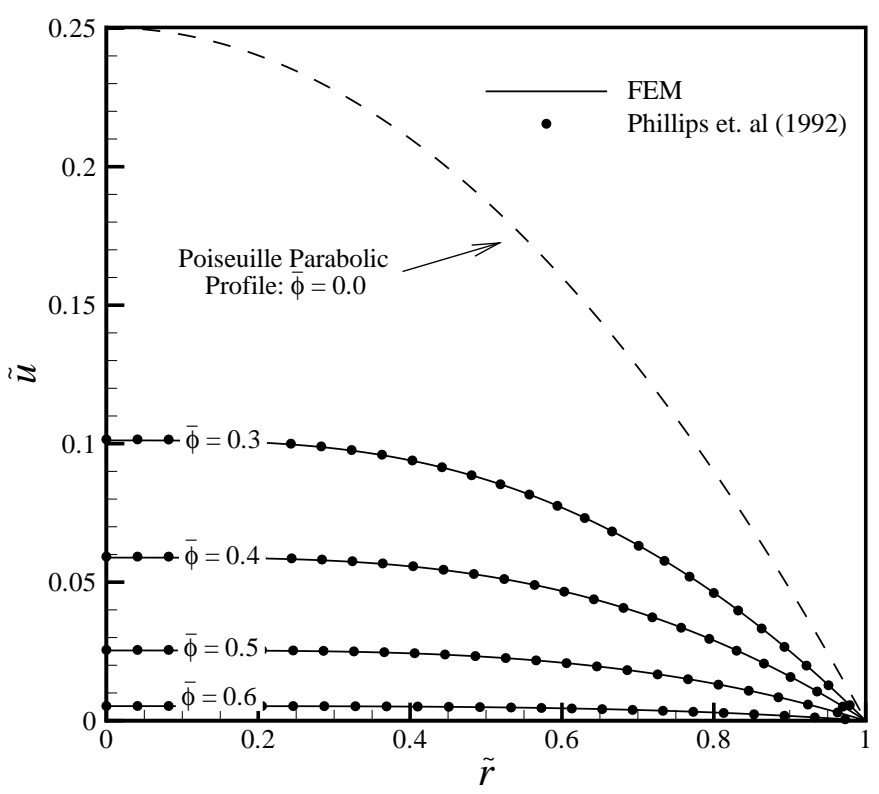

Figure 4.2: Comparison between the numerical results of the velocity profiles with Phillips' analytical solutions. The parabolic profile of a typical Poiseuille flow (i.e. $\bar{\phi}=0.0$ ) is plotted as reference. 


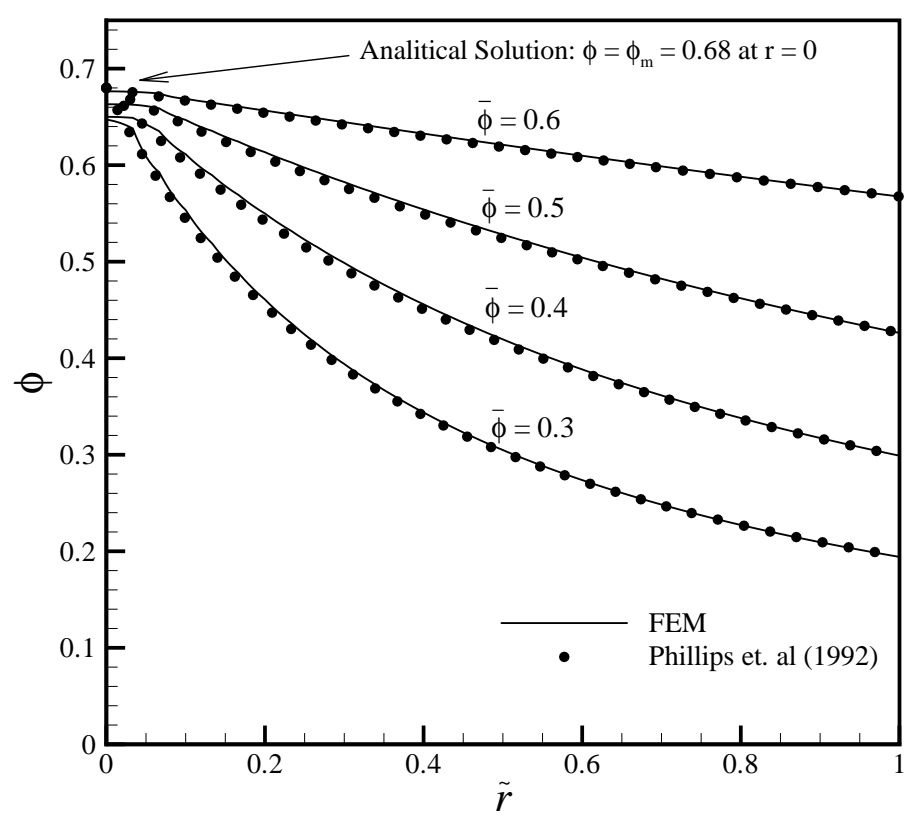

Figure 4.3: Comparison between the numerical results of the concentration profiles with Phillips' analytical solutions.

Figure 4.4 shows the contours of particle concentration for $\bar{\phi}=0.4$ and 0.6, respectively. From this results one can observe that at the entrance (i.e., $x=0 \mathrm{~mm}$ ) the concentration is uniform and equal to the corresponding bulk concentration, as imposed. From the latter region until, approximately, $X=6.0 \mathrm{~mm}$ the concentration profile develops and from this position to the end of the computational domain, the flow is almost fully-developed. It is important to highlight that, although Phillips et al. (119.92) stated that the diffusion coefficients $k_{c}$ and $k_{\eta}$ should be $\mathcal{O}(1)$, in this work we employed high values of those parameters (e.g., $k_{c}=32.8$ and $k_{\eta}=49.6$ ) in order to enhance particle migration mechanisms, reduce the development entrance length of the particle concentration profile and maintain a constant ratio of $k_{c} / k_{\eta}=0.66$. Also, note that the profiles depicted in Figs. 4.3 and 4.2 were taken from the final section of the tube at $X=10.0 \mathrm{~mm}$. 

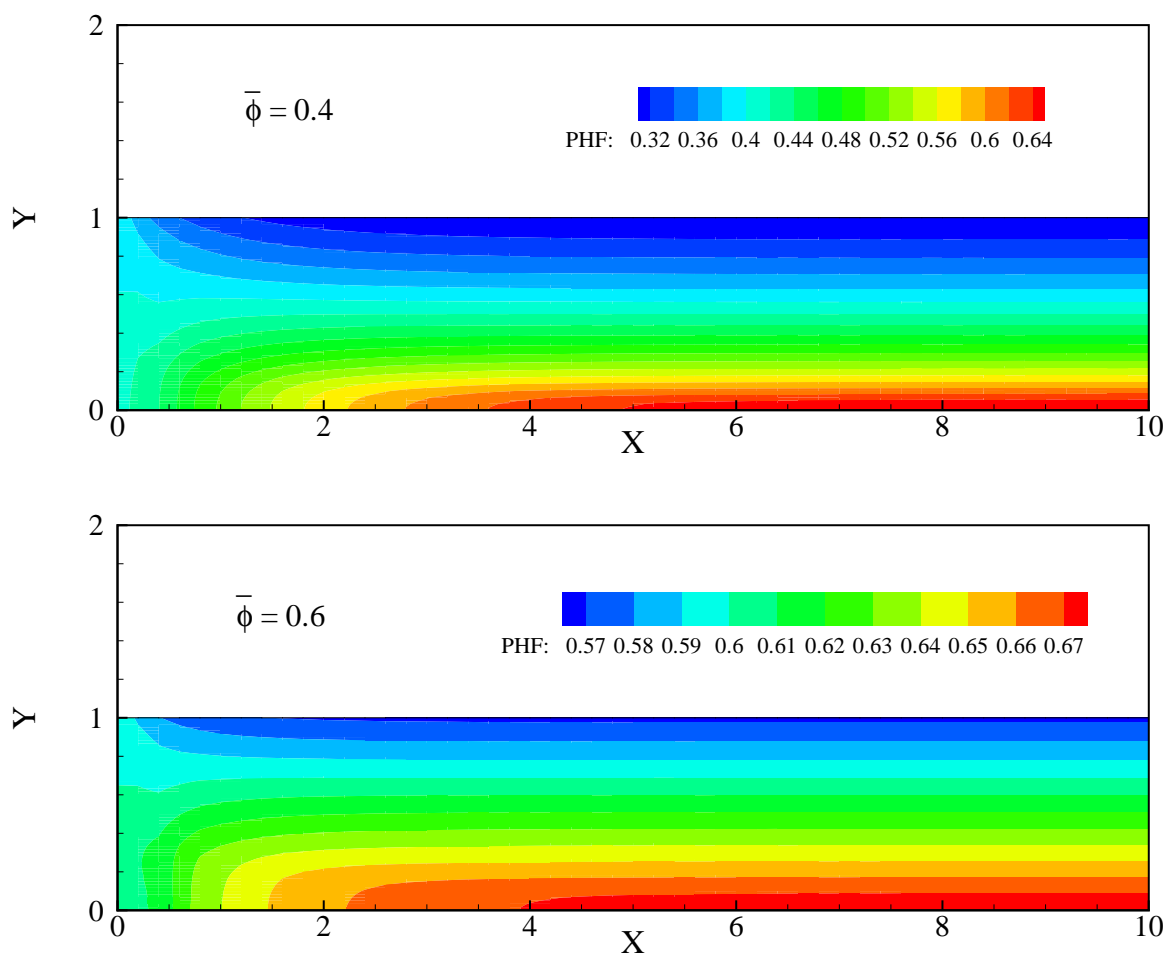

Figure 4.4: Countors for bulk concentrations 0.40 and 0.60, respectively.

\section{2}

\section{Slot Coating Analysis}

This section presents the results of a slot coating flow in which the coating liquid is a suspension of rigid and spherical particles. As stated before, in applications where particle size is small enough so that inter-particle forces compete with macroscopic contributions of the flow, the suspension becomes shear sensitive. First, a mesh convergence test is performed in an effort to define the optimum mesh for this work. After that, an analysis of particle concentration profiles considering migration Models I, II and III is presented for a final film thickness equal to half of the coating gap. In addition to that, a similar investigation is employed with regards to a smaller film thickness considering three different Capillary numbers. In this work, the Capillary number is evaluated based on the bulk viscosity of the suspension. Finally, the effect of particle migration and shear sensitive behavior on the low-flow limit study is evaluated. All the simulations where done considering a bulk concentration $\bar{\phi}=0.30$ and particle size $a=1 \mu \mathrm{m}$. 


\subsection{1}

\section{Mesh convergence test}

In order to determine the optimum mesh to be used in this work, the convergence of the solution of a typical slot coating flow of a shear-sensitive particle suspension was tested for three different meshes. The following meshes are shown in Figs. 4.5, 4.6 and 4.7 and the main difference between them is the number of elements in the downstream region where the final film is deposited. Tables 4.1, 4.2 and 4.3 show the number of elements per regions in each mesh following the same numbering presented in Figure $3 . \mathrm{C}$. In this sense, Mesh 1 is composed by 328 elements, with 9,621 unknowns; Mesh 2 is composed by 418 elements, with 12, 135 unknowns; and Mesh 3 is composed by 608 elements with 17, 405 unknowns.

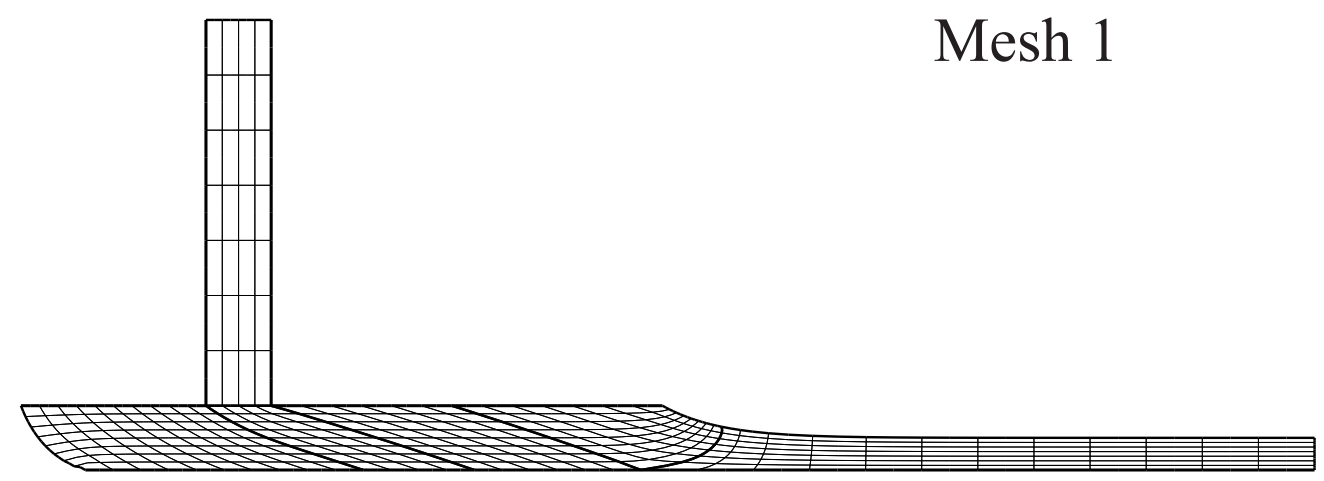

Figure 4.5: Representative Mesh 1 used to discretize the problem: 328 elements and 9,621 unknowns.

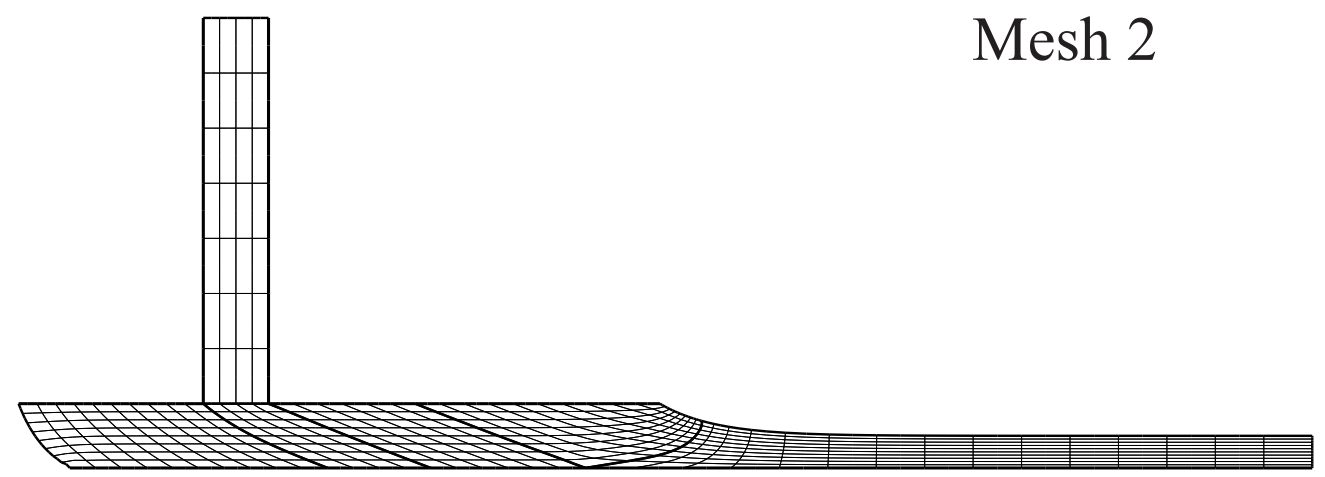

Figure 4.6: Representative Mesh 2 used to discretize the problem: 418 elements and 12,135 unknowns. 


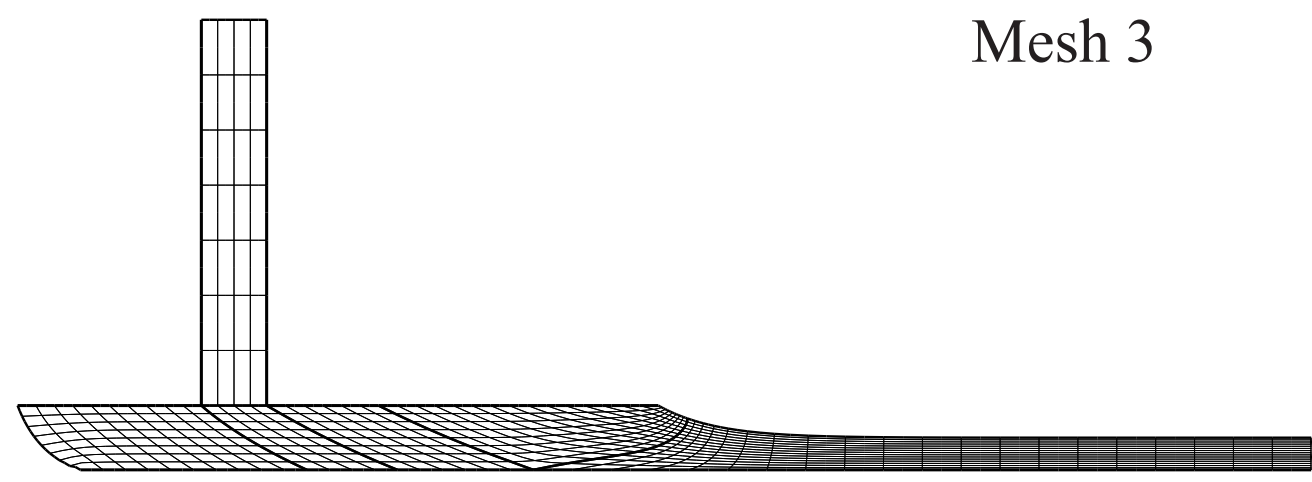

Figure 4.7: Representative Mesh 3 used to discretize the problem: 608 elements and 17, 405 unknowns.

Table 4.1: Number of elements in each region of Mesh 1.

\begin{tabular}{|c|c|c|}
\hline Region & y-direction & x-direction \\
\hline \hline 1 & 8 & 10 \\
\hline 2 & 8 & 4 \\
\hline 3 & 7 & 4 \\
\hline 4 & 8 & 6 \\
\hline 5 & 12 & 7 \\
\hline 6 & 8 & 7 \\
\hline
\end{tabular}

Table 4.2: Number of elements in each region of Mesh 2.

\begin{tabular}{|c|c|c|}
\hline Region & y-direction & x-direction \\
\hline \hline 1 & 8 & 10 \\
\hline 2 & 8 & 4 \\
\hline 3 & 7 & 4 \\
\hline 4 & 8 & 6 \\
\hline 5 & 15 & 10 \\
\hline 6 & 8 & 10 \\
\hline
\end{tabular}

Table 4.3: Number of elements in each region of Mesh 3.

\begin{tabular}{|c|c|c|}
\hline Region & y-direction & x-direction \\
\hline \hline 1 & 8 & 10 \\
\hline 2 & 8 & 4 \\
\hline 3 & 7 & 4 \\
\hline 4 & 8 & 6 \\
\hline 5 & 20 & 15 \\
\hline 6 & 8 & 15 \\
\hline
\end{tabular}


The convergence of the solution was tested by comparing the numerical predictions for particle concentration profiles of each mesh at the final section of the deposited film. For this purpose, we assumed the following operating parameters: velocity of the substrate $V=0.4 \mathrm{~m} / \mathrm{s}$; bulk concentration $\bar{\phi}=$ 0.30 ; vacuum pressure $P_{v a c}=-3.5 \mathrm{kPa}$; and final film thickness $t=H / 2$, where $H=100 \mu \mathrm{m}$ is the coating gap. Also, we employed particle migration Model III which incorporates all the modifications of the original Phillips model accounted in this work along with the viscosity model of Cross, i.e. $\eta=\eta(\phi, \dot{\gamma})$, with solvent viscosity $\eta_{s}=0.02 \mathrm{~Pa}$ s.

As an initial analysis, minimum and maximum local particle concentrations for each mesh were computed considering the whole domain and organized in Table 4.4. Note that all the values are approximately the same with an agreement $\mathcal{O}\left(10^{-2}\right)$ which shades light to a convergence of the solution of interest. With this in mind, in an effort to enhance the investigation and subsequent choice of the mesh, particle concentration profiles were plotted along the final film thickness as shown in Fig. 4.8. From these results one can observe that the solutions are virtually the same and converge to a master particle concentration curve.

Table 4.4: Minimum and maximum local concentrations for each mesh.

\begin{tabular}{|c|c|c|}
\hline Mesh & $\phi_{\min }$ & $\phi_{\max }$ \\
\hline \hline 1 & 0.1757 & 0.4912 \\
\hline 2 & 0.1765 & 0.4913 \\
\hline 3 & 0.1778 & 0.4915 \\
\hline
\end{tabular}

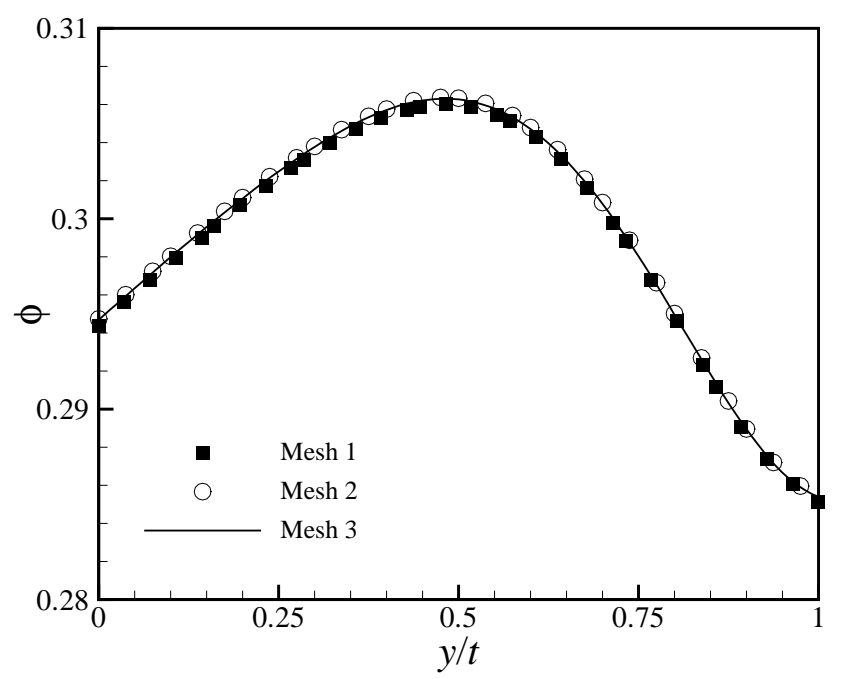

Figure 4.8: Predictions using three different meshes of the particle concentration profile at the exit of the deposited film. 
In order to select the most appropriate mesh, we integrated numerically the numerical particle concentration profile along the final film thickness for each mesh and evaluated the error in relation to the bulk concentration. Since there are no source and/or sink terms in the particle migration equation, the latter integral should recover the bulk concentration under the light of conservation principles. Thereupon, the relative errors for each mesh are: Mesh $1=1.801 \%$; Mesh $2=0.236 \%$; and, finally, Mesh $3=0.896 \%$, which indicates that the solution is mesh independent considering the set of meshes under investigation. In this sense, the predictions presented in this work were obtained by using Mesh 2 since it presents the lower relative error.

\subsection{2}

\section{Effect of shear sensitivity}

In this subsection, the focus is to investigate the effects of considering or not a shear-sensitive viscosity model in cases where particle size is small enough so that colloidal forces are relevant. Thereupon, the following results were evaluated using the viscosity model of Cross in comparison with the wellknown viscosity model of Krieger. Note that, Krieger's model is sensitive to local particle concentration only, i.e. $\eta=\eta(\phi)$, and that, on the other hand, Cross' model is sensitive to local particle concentration and local shear rate, so that $\eta=\eta(\phi, \dot{\gamma})$.

Initially, the flow field is obtained numerically by solving the governing equations presented in Chapter 2 for suspensions of spherical and rigid particles neglecting any kind of migration mechanisms. This is possible by setting the diffusion coefficients $k_{c}$ and $k_{\eta}$ equal to zero. In this case, the suspension can be understood as a Newtonian liquid with a constant viscosity throughout the entire domain, and thereby, $\eta=\eta_{0}(\bar{\phi})$. It is important to recall that $\eta_{0}$ is the viscosity evaluated at the first Newtonian plateau and it is used as a reference in this work. Figure 4.9 shows the contour of particle concentration for a bulk concentration $\bar{\phi}=0.30$, vacuum pressure $P_{v a c}=-3.5 \mathrm{kPa}$, ambient pressure $P_{a t m}=0 \mathrm{kPa}$, velocity of the substrate $V=400 \mathrm{~mm} / \mathrm{s}$, interfacial tension $\sigma=60 \mathrm{dyn} / \mathrm{cm}$, dynamic contact angle $\theta_{D C L}=40^{\circ}$ and final film thickness $t=H / 2$ (i.e., $t=50 \mu \mathrm{m}$ ). Moreover, the bulk Capillary number for this simulation was $C a=0.25$. Under those conditions, the numerical parameter employed to overcome the singularity of the DFM was set to $\delta \approx 10^{-3}$. 


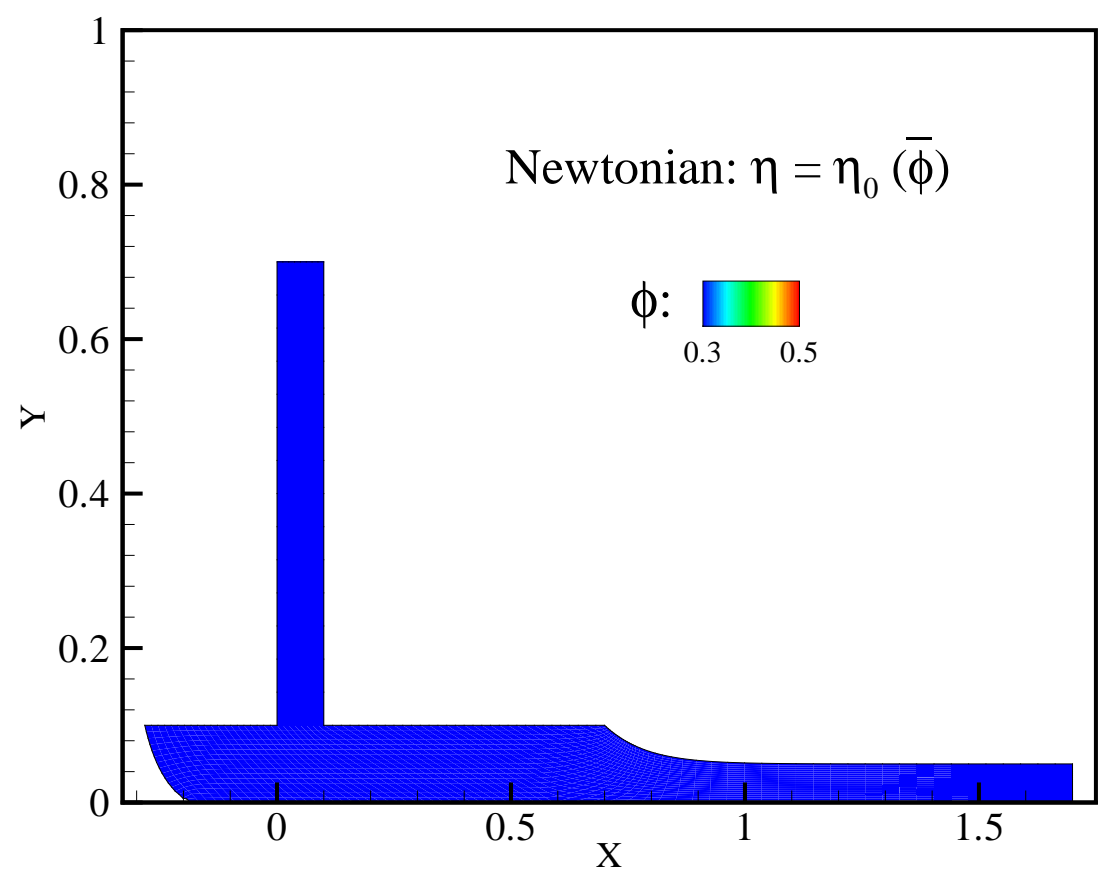

Figure 4.9: Contour of particle concentration for a suspension in which the viscosity is a constant function of the bulk concentration, $\bar{\phi}=0.30$.

The next step was to consider particle migration mechanisms in the simulations using a fixed ratio of the phenomenological parameters $k_{c}^{P} / k_{\eta}^{P}=0.66$ for Models I, II and III. We set $k_{c}^{P}=32.8$ and $k_{\eta}^{P}=49.6$ to enhance the effect of particle migration. All other parameters of the flow such as vacuum pressure and final film thickness remained the same as the Newtonian simulation which, in turn, was used as an initial guess for simulations considering particle migration. This part of the work investigates particle concentration contours and profiles at the final section of the deposited film at $X=1.7 \mathrm{~mm}$ and the position of the upstream meniscus considering both viscosity models under study: Krieger and Cross, respectively.

Figures $4.10,4.17$ and 4.22 show the contours of particle concentration considering the three migration models and the viscosity relation of Krieger. It is clear that particles tend to migrate from regions of high to low shear rates. For example, this feature is evident near the exit of the feed slot $(Y \approx 0.2$ $\mathrm{mm})$. There are regions of high shear rates near the walls and regions of lower shear rates at the vicinities of the center line. In this sense, due to overall contributions of particle collision frequency, which scales with $\phi \dot{\gamma}$, and by means of gradients in viscosity, the particles tend to migrate preferably towards the center of the feed slot where local particle concentration reaches 
values approximately equal to $\phi=0.4902$ for Model I; $\phi=0.4897$ for Model II; and $\phi=0.3878$ for Model III. In the feed slot region, since there is no curvature of the streamlines, Models I and II are the same and should recover the same values of local particle concentration which happens indeed. Small deviations between Models I and II shall regarded to particle migration contributions at $Y=0.1 \mathrm{~mm}$ where curvature-induced migration is important. At the same time, Model III differs considerably from Models I and II since it considers that the ratio $k_{c} / k_{\eta}$ is a function of local particle concentration and this dependency is explicit on $k_{c}$ which is the diffusion parameter related to spatially varying interacting frequency migration. Again, following the results presented by Kim et al. (2008), since $k_{c}=1.5 \phi k_{c}^{P}, k_{\eta}=k_{\eta}^{P}$ and $k_{\kappa}=0.75 k_{c}^{P}$, the spatially varying interaction frequency is lower and the contribution of the migration due to gradients in viscosity is greater and, hence, the flux of particles from regions of high to low viscosities is also magnified. This latter analysis explains the reason why Model III presents lower concentrations at the center of the feed slot in comparison with Models I and II.

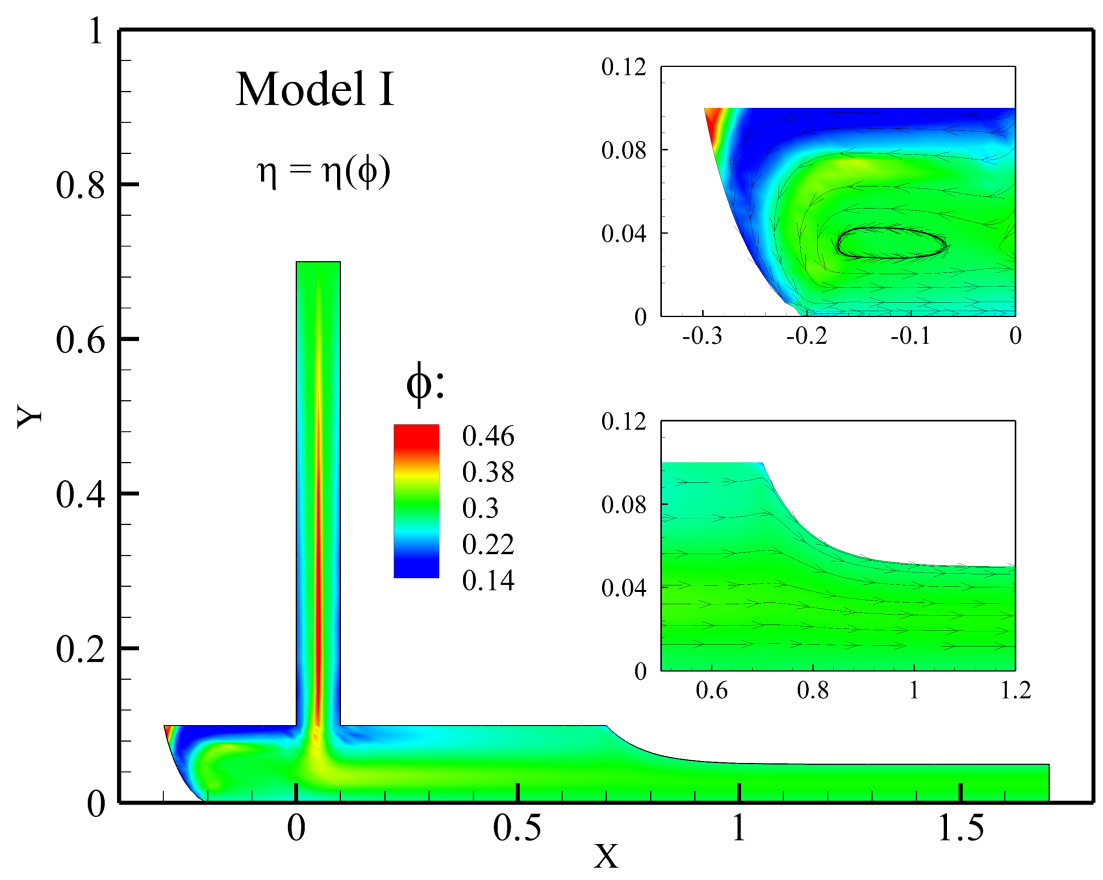

Figure 4.10: Contour map of particle concentration of the upstream region considering migration Model I and the viscosity model of Krieger. 


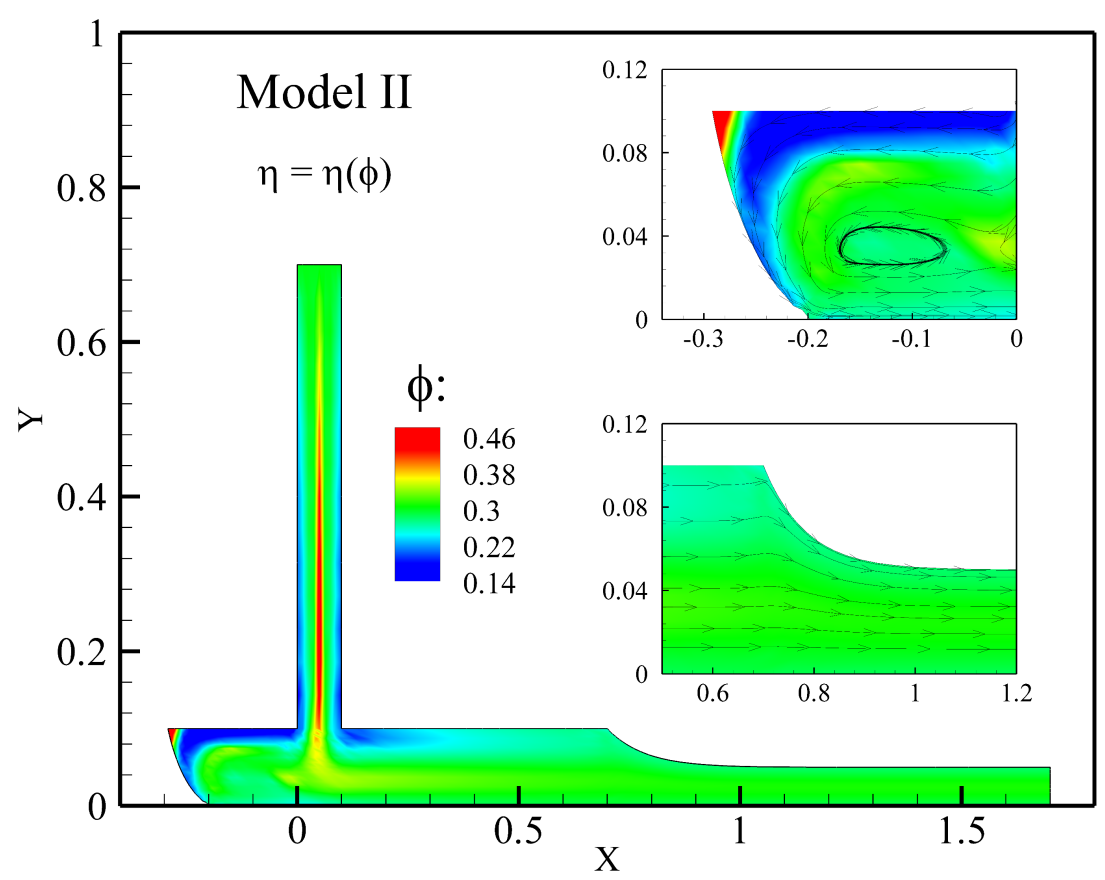

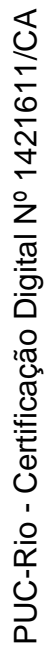

Figure 4.11: Contour map of particle concentration of the upstream region considering migration Model II and the viscosity model of Krieger.

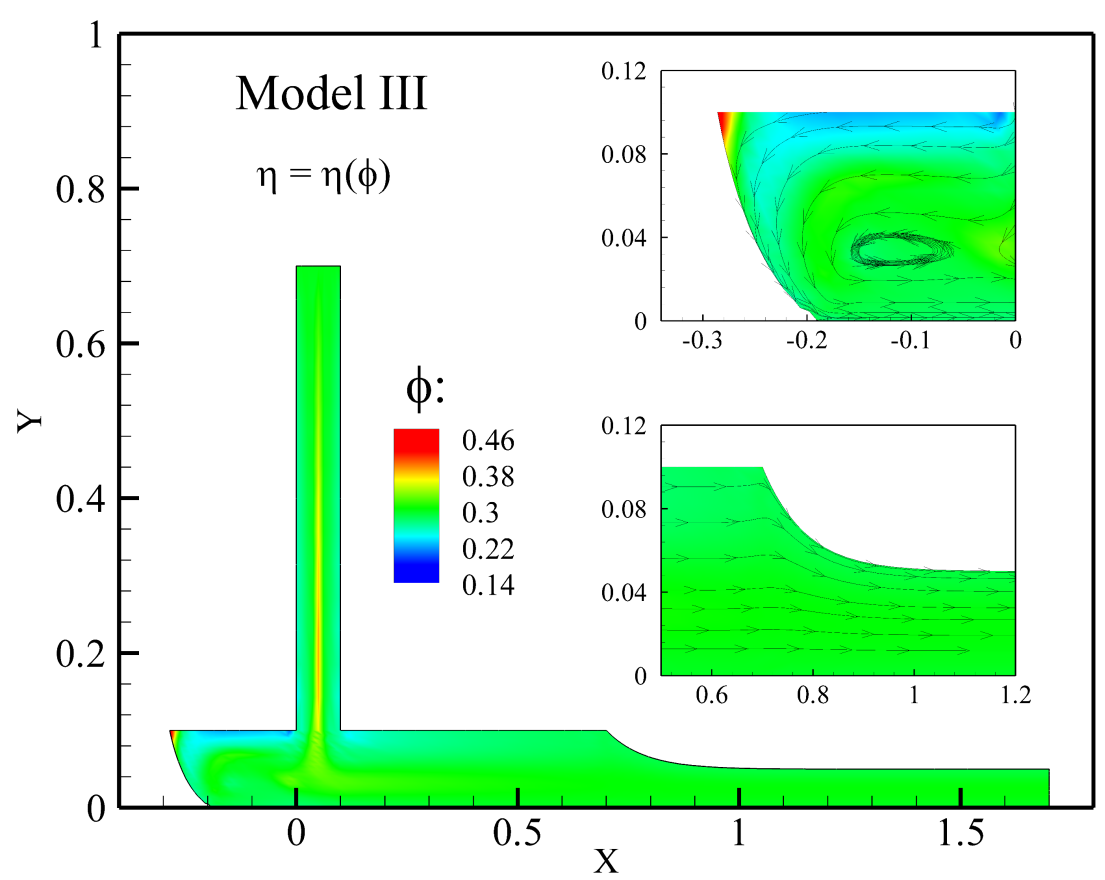

Figure 4.12: Contour map of particle concentration of the upstream region considering migration Model III and the viscosity model of Krieger. 
Now, making the transition from the feed slot to the upstream region, it is clear that particles move away from the die lip towards the region of high shear rate. Particle migration due to the curvature of the streamlines also becomes relevant. Recall from Eq. 2.28 that curvature-induced migration drives the particles in the same direction as the local normal vector to the streamline, $\boldsymbol{n}_{\boldsymbol{S T}}$. As a consequence, it is expected that regions opposing curved streamlines should present an increment in local particle concentration, especially, when comparing Models I and II. Since Model III incorporates a concentration dependency on the ratio $k_{c} / k_{\eta}$, the magnitude of curvature-induced particle migration shall decrease as $k_{\kappa}=k_{c}$ and $k_{c}=k_{c}(\phi)$. Indeed, these assumptions are confirmed and can be seen at Figs. 4.10, 4.17 and 4.72 where the local particle concentration at the vicinities of the upstream static contact line for Models I, II and III are 0.4695, 0.4844 and 0.4325, respectively.

Following the same track, the curvature of the streamlines at the exit of the feed slot and at the beginning of the downstream meniscus induces a migration towards the substrate and this behavior explains the difference between the particle concentration profiles at the final section of the deposited film for Models I, II and III depicted in Figure 4. I3]. Moreover, since migration Model III incorporates a concentration-dependent phenomenological parameter $k_{c}=k_{c}(\phi)$, then the migration mechanism due to spatially varying viscosity has a greater contribution so that more particles tend to migrate from regions of high to low viscosity in an opposing flux due to gradients in shear rate. This latter analysis explains why the concentration at the center of the final film is lower and at the extremes higher for Model III in contrast to Models I and II. As the coating liquid is deposited at the substrate, particle concentration profile is convected by the flow. 


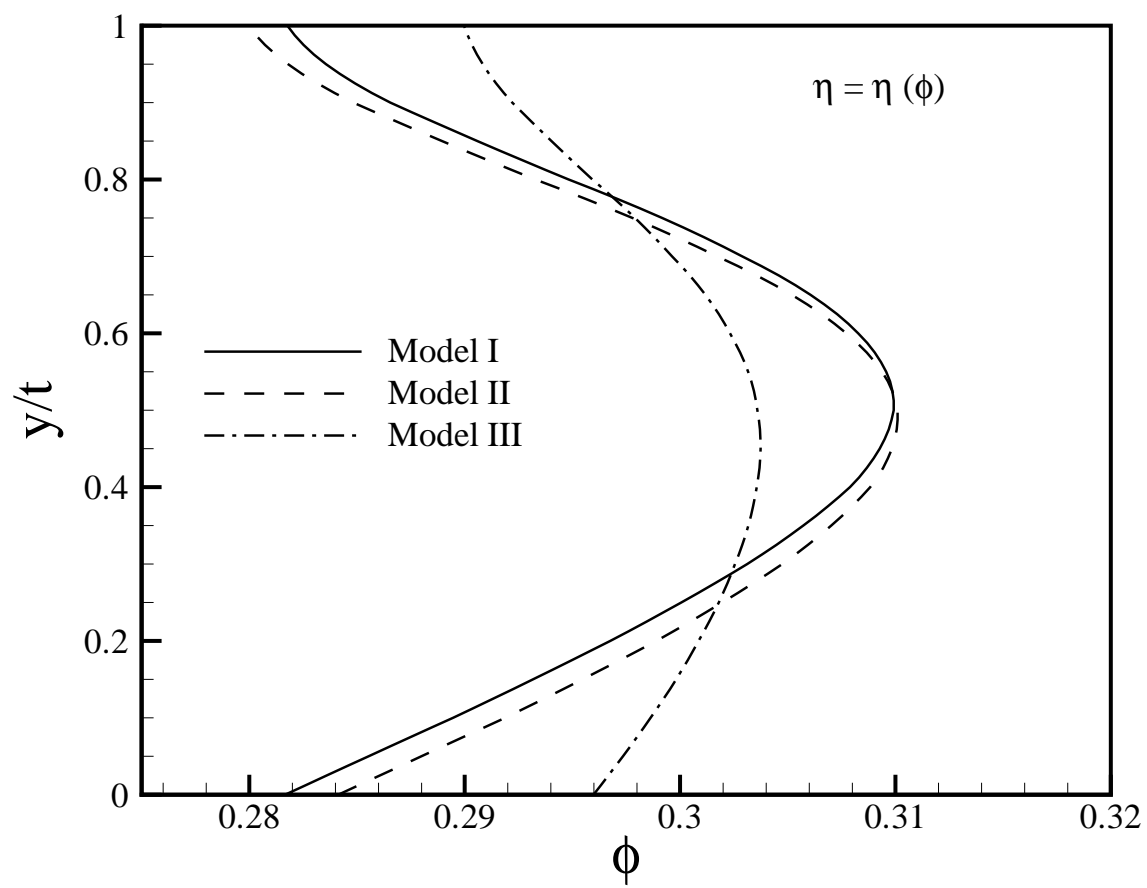

Figure 4.13: Particle concentration profiles the deposited film (i.e., $X=1.7$ $\mathrm{mm}$ ) for a viscosity model $\eta=\eta(\phi)$ and for migration Models I, II and III.

In order to verify if shear sensitive behavior affects the flow, an examination of the contours of concentration, positions of the upstream dynamic contact line and profiles of particle concentration at the deposited film upstream is performed. First of all, Figs. 4.15, 4.16 and 4.17 show the contour maps for migration Models I, II and III. Note that, comparing these results with Figures $4.10,4.01$ and 4.5 it is possible to detect that the behavior of particle migration is qualitatively the same, however, the position of the downstream dynamic contact line is different.

Figure 4.14 illustrates the shift of the upstream meniscus in comparison to the Newtonian reference for all migration models and both types of viscosity models under study. Since particles tend to migrate towards regions of low shear rates, they move away from the upstream die lip and the flow is lubricated is this region. As a result, the upstream meniscus shifts and this movement is even greater when the suspension is shear thinning as the local viscosity reduces with shear rate. To demonstrate this, let $Q_{U}$ be the flow rate under the upstream die for a fixed gap width. The flow rate must be zero in this region in order to conserve the mass. Since the pressure driven flow rate scales with $\left(\Delta P_{U} / L\right) / \eta$, and assuming that the pressure difference at the upstream region is approximately constant over a distance $L$, if the suspension is shear thinning, than, in order to maintain the zero flow rate, the size of the upstream 
region must increase which happens indeed. In fact, the $\mathrm{X}$-coordinate of the dynamic contact line for a Newtonian fluid with $\eta=\eta(\bar{\phi})$ is $-0.185 \mathrm{~mm}$ and Table 4.5 shows the respective X-coordinates of the upstream meniscus for Models I, II and III.

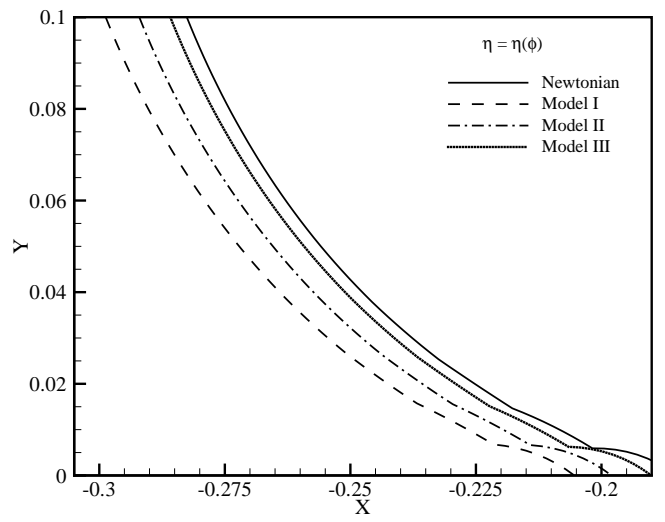

$4.14(\mathrm{a})$

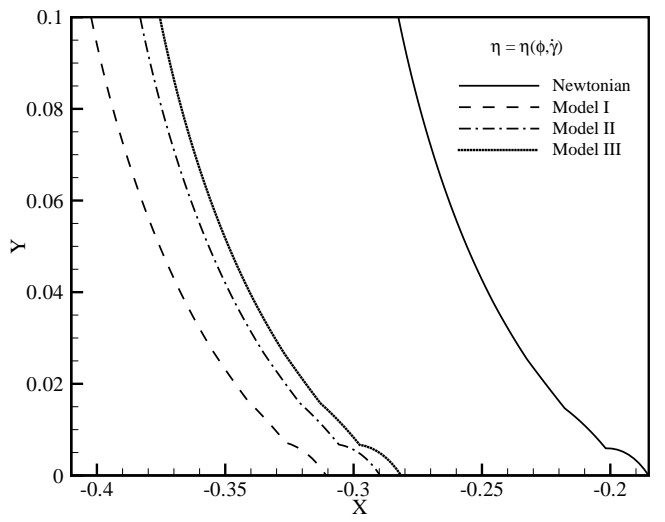

$4.14(\mathrm{~b})$

Figure 4.14: Graphs of the position and shape of the upstream meniscus when $\bar{\phi}=0.30$ : (a) using Krieger's viscosity model and (b) using Cross' model.

Table 4.5: X-coordinate of the dynamic contact line related to migration models I, II and III.

\begin{tabular}{|c|c|c|}
\hline Model & $X_{D C L}[\mathrm{~mm}]-\eta(\phi)$ & $X_{D C L}[\mathrm{~mm}]-\eta(\phi, \dot{\gamma})$ \\
\hline \hline I & -0.206 & -0.311 \\
\hline II & -0.198 & -0.290 \\
\hline III & -0.190 & -0.282 \\
\hline
\end{tabular}

The reason why Models I, II and III do not predict the same meniscus curve for each viscosity model is that Model II incorporates a curvature-induced migration which drives the particles towards the upstream free surface which generates a greater local viscosity in this region in comparison to Model I. A similar trend is verified for Model III in which the overall concentration under the upstream lip is slightly uniform so that a greater resistance to flow is verified leading to an also higher local viscosity. The latter analysis can be applied for both viscosity models with the respective quantitative differences.

Following the same analysis made for a concentration dependent viscosity with regards to curvature-induced migration, one can observe that the particles tend to migrate in the direction of the normal vector to the streamline as illustrated in Figs. 4.15, 4.16 and 4.17. It is important to highlight that local particle concentration at the vicinities of the upstream static contact line is greater for all models of migration considering Cross' viscosity model in 
contrast to Krieger's one. In this case, the values of local concentration at the vicinities of the upstream static contact line for Models I, II and III are 0.5284, 0.5319 and 0.4582 , respectively. A plausible reason is that more particles tend to occupy the upstream region since the size of this part is augmented when shear-thinning effects are taken into account.

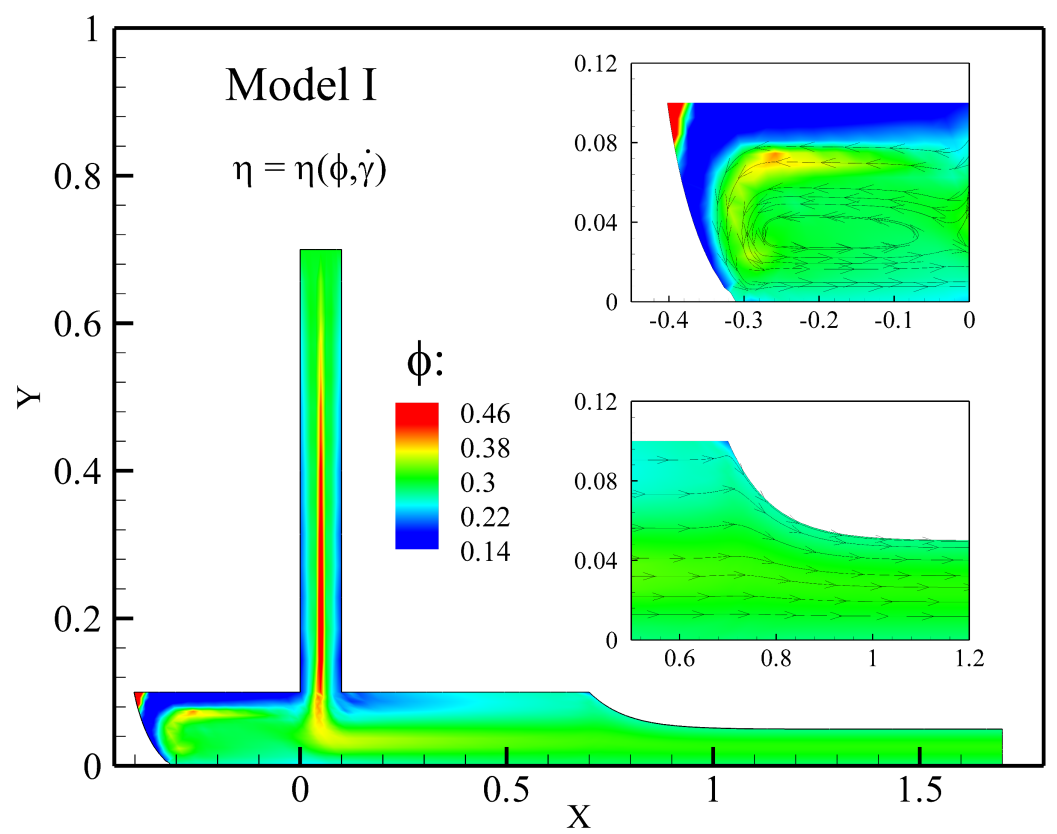

Figure 4.15: Contour map of particle concentration considering migration Model I and the viscosity model of Cross.

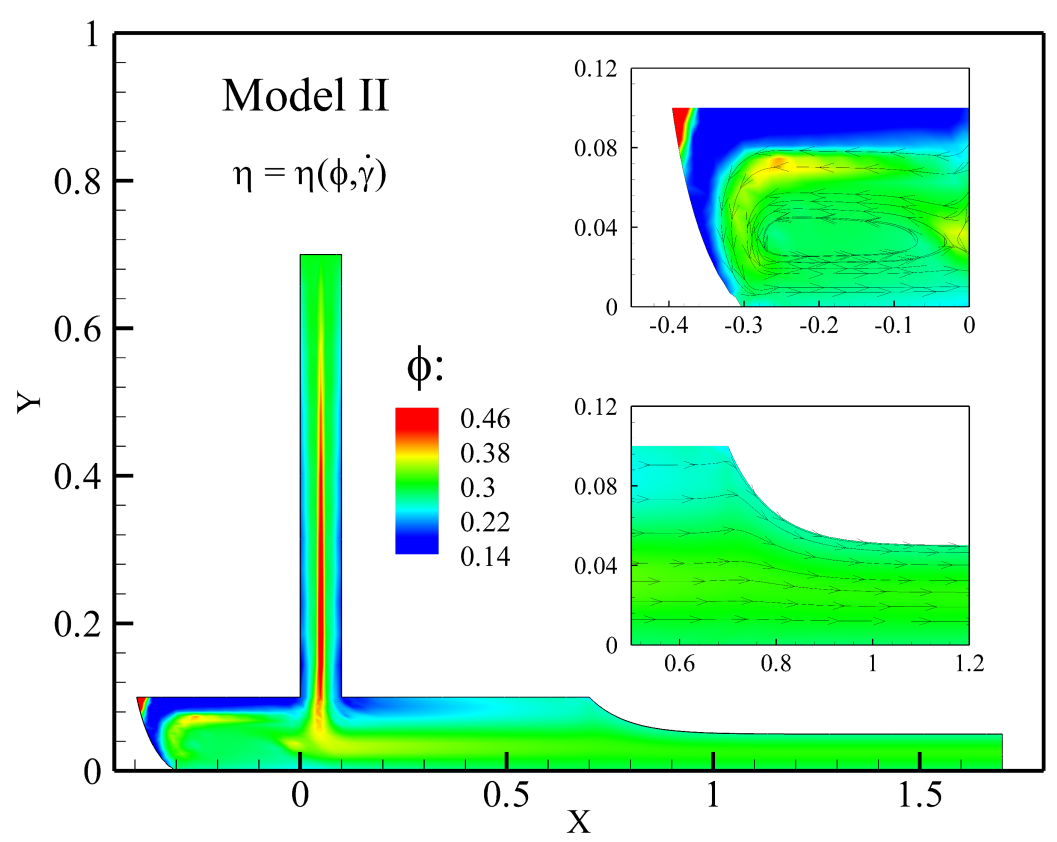

Figure 4.16: Contour map of particle concentration considering migration Model II and the viscosity model of Cross. 


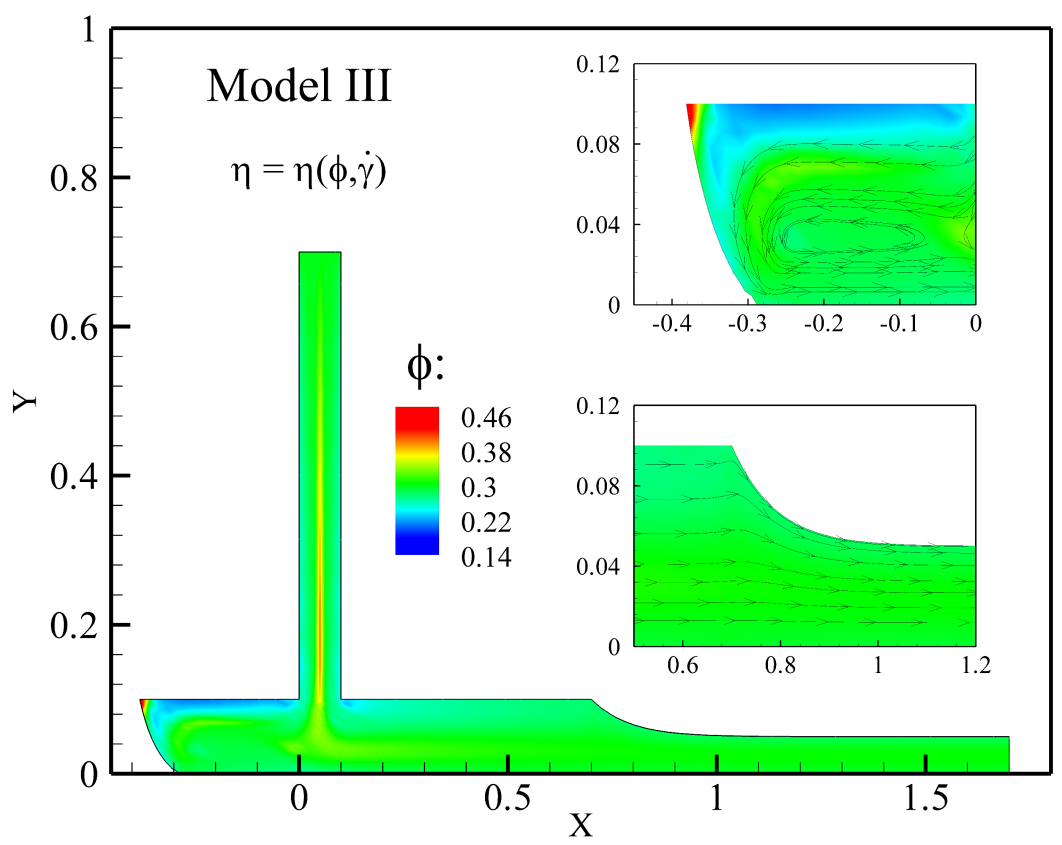

Figure 4.17: Contour map of particle concentration considering migration Model III and the viscosity model of Cross.

In addition to that, particle concentration profiles at the deposited film are shown in Fig. 1.18. Again, the results are qualitatively in agreement with the results of Fig. 4.13 and it is possible to see that local particle concentration at the center of the film is higher for a shear sensitive suspension model. This can be explained by the fact that the shear stress at the die lip walls should remain the same for both viscosity models since the flow rate is also equal. Moreover, in these regions local shear rate reaches its maximum value leading to a shear-thinning behavior. Since $\tau_{w}=\eta \dot{\gamma}$, if the viscosity decreases, then the local shear rate must increase in order to maintain a fixed value of $\tau_{w}$. In this manner, for a shear sensitive coating liquid, gradients in shear rate are magnified in comparison with a concentration-dependent viscosity model so that the migration mechanism due to spatially variations in collision frequency is also augmented. Therefore, more particles tend to migrated towards regions of lower shear rate reducing the concentration at the wall and increasing it in lower shear rate regions such as the center of the feed slot. Then, for a film thickness of $H / 2$ one shall observe that the shear rate under the downstream die is practically uniform so that the overall contributions of particle migration mechanisms are weaker. As a result, the shape of the particle concentration profile that leaves the feed slot is convected to the film formation which explains the format of the results presented in Figs. 4.18 and 4.13 . 


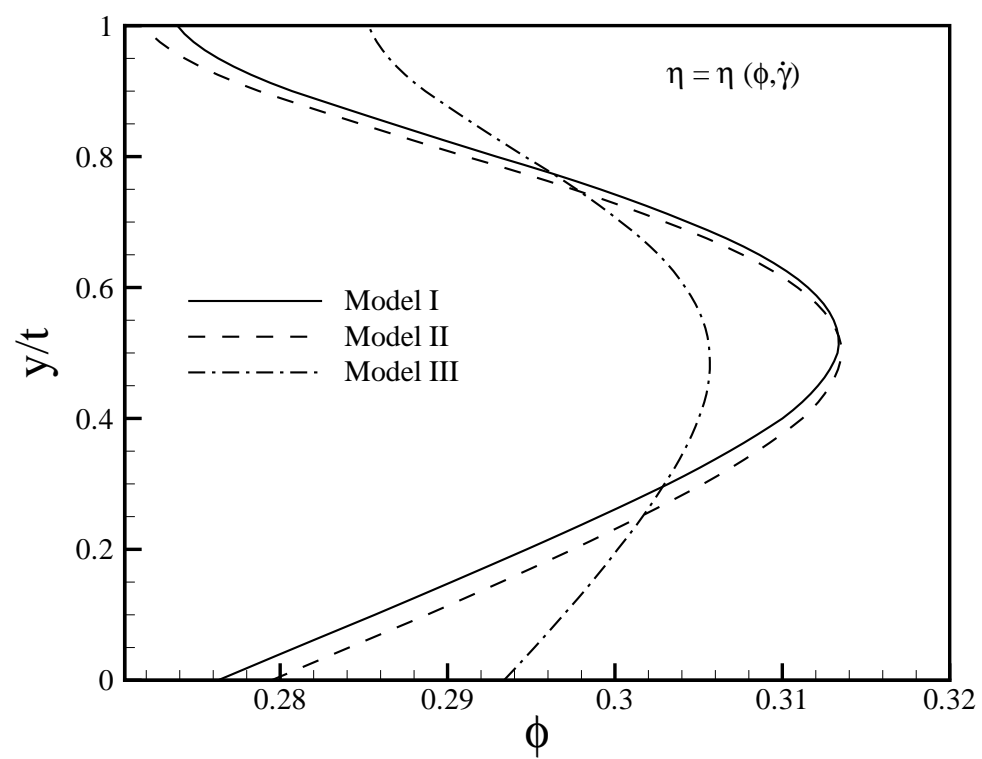

Figure 4.18: Particle concentration profiles at the final section of the deposited film (i.e., $X=1.7 \mathrm{~mm}$ ) for a suspension in which the viscosity is a function of local particle concentration only, $\eta=\eta(\phi, \dot{\gamma})$ and for Models I, II and III.

\subsection{3}

\section{Effects of Capillarity}

In this part of the results the goal is to explore how changes in the Capillary number affects particle concentration profiles at the final deposited film and the shape of the downstream meniscus. Note from the dimensionless form of the particle transport equation given by Eq. ([2.39), that there is no explicit dependency on the substrate velocity $V$ so that changes in Capillary number, performed by changing $V$, maintain the same balance between convective and diffusive contributions. Also, since the interfacial tension is assumed to be constant in this work, variations in the Capillary number are solely regarded to viscous effects. As the viscosity model depends on the local particle concentration which also depends on operating conditions, viscous effects were modeled considering the viscosity of the suspension as a function of the bulk concentration only so that $C a=\eta_{0}(\bar{\phi}) \mathrm{V} / \sigma$.

All simulations were held considering three different operating velocities of the substrate and hence Capillary numbers. In all cases the numerical parameters were: final film thickness $t=35 \mu \mathrm{m}$; stabilizing parameter $\delta=$ $6 \times 10^{-2}$; bulk concentration $\bar{\phi}=0.30$; fixed ratio of diffusion parameters $k_{c}^{P} / k_{\eta}^{P}=0.66$; interfacial tension $\sigma=60 \mathrm{dyn} / \mathrm{cm}$; dynamic contact angle $\theta_{D C A}=40^{\circ}$ and ambient pressure $P_{a t m}=0 \mathrm{kPa}$. The vacuum pressure was adjusted in order to keep the upstream meniscus away from the feed slot and 
consequently a stable coating bead. In addition to that, an examination of both viscosity models (i.e., Krieger and Cross) considering migration Models I, II and III was performed. At this point, it is important to make clear that, for a concentration-dependent viscosity approach, only Model I was considered in the subsequent investigation since an emphasis on shear-sensitive coating liquids is the main concern herein.

As an illustration, Figs. 4.19, 4.20, 4.27 and 4.22 show the contours of local particle concentration considering migration Models I and II and both viscosity relations for three distinct Capillary numbers. Table 4.6 presents the vacuum pressure used for each Capillary number in order to obtain a stable coating bead. Note, again, that for a shear sensitive liquid the upstream region is longer than the corresponding one for a suspension which behaves as a generalized Newtonian fluid with a concentration-dependent viscosity model.

Table 4.6: Capillary numbers and adjustable parameters for a stable slot coating flow considering three cases.

\begin{tabular}{|c|c|c|c|}
\hline Case & $V$ & $C a$ & $P_{v a c} H / \sigma$ \\
\hline \hline I & $0.1 \mathrm{~m} / \mathrm{s}$ & 0.077 & -3.33 \\
\hline II & $0.5 \mathrm{~m} / \mathrm{s}$ & 0.386 & -11.67 \\
\hline III & $1.5 \mathrm{~m} / \mathrm{s}$ & 1.158 & -30.0 \\
\hline
\end{tabular}

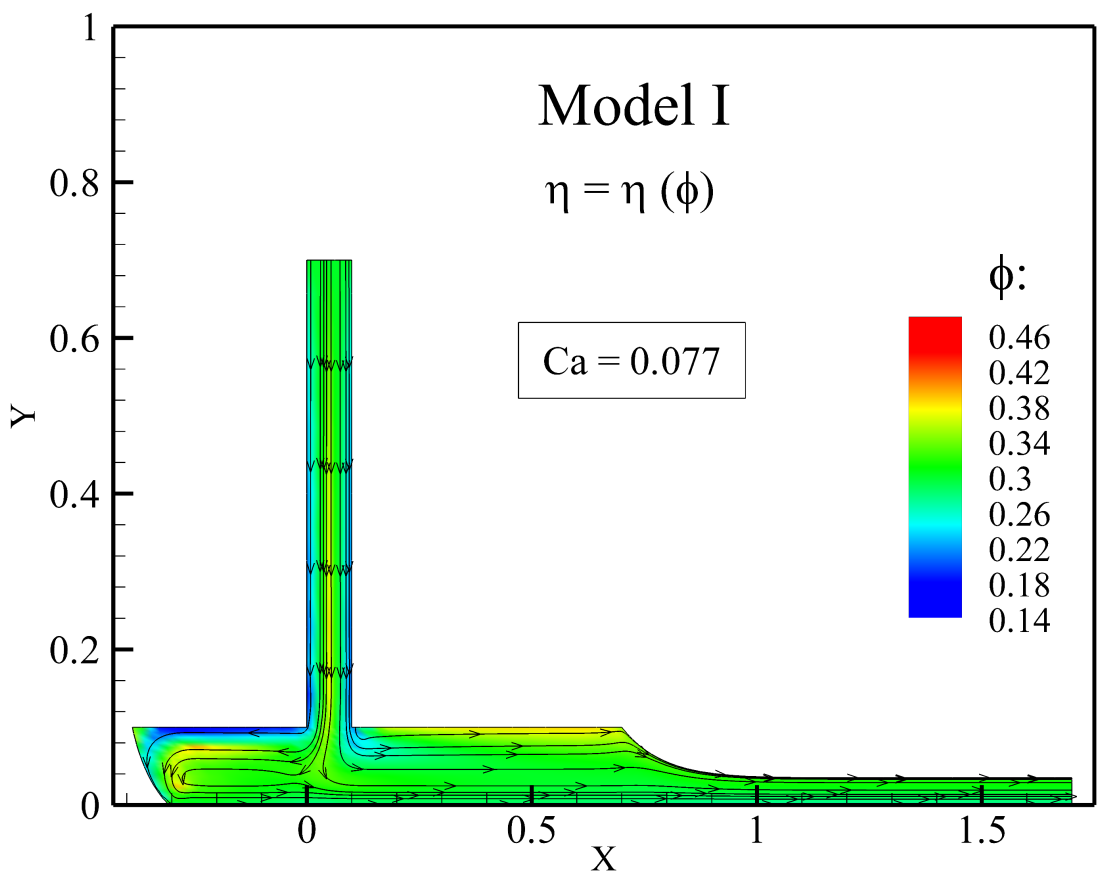

Figure 4.19: Contours of particle concentration for Capillary number 0.077 using Krieger's viscosity model and migration Model I. 


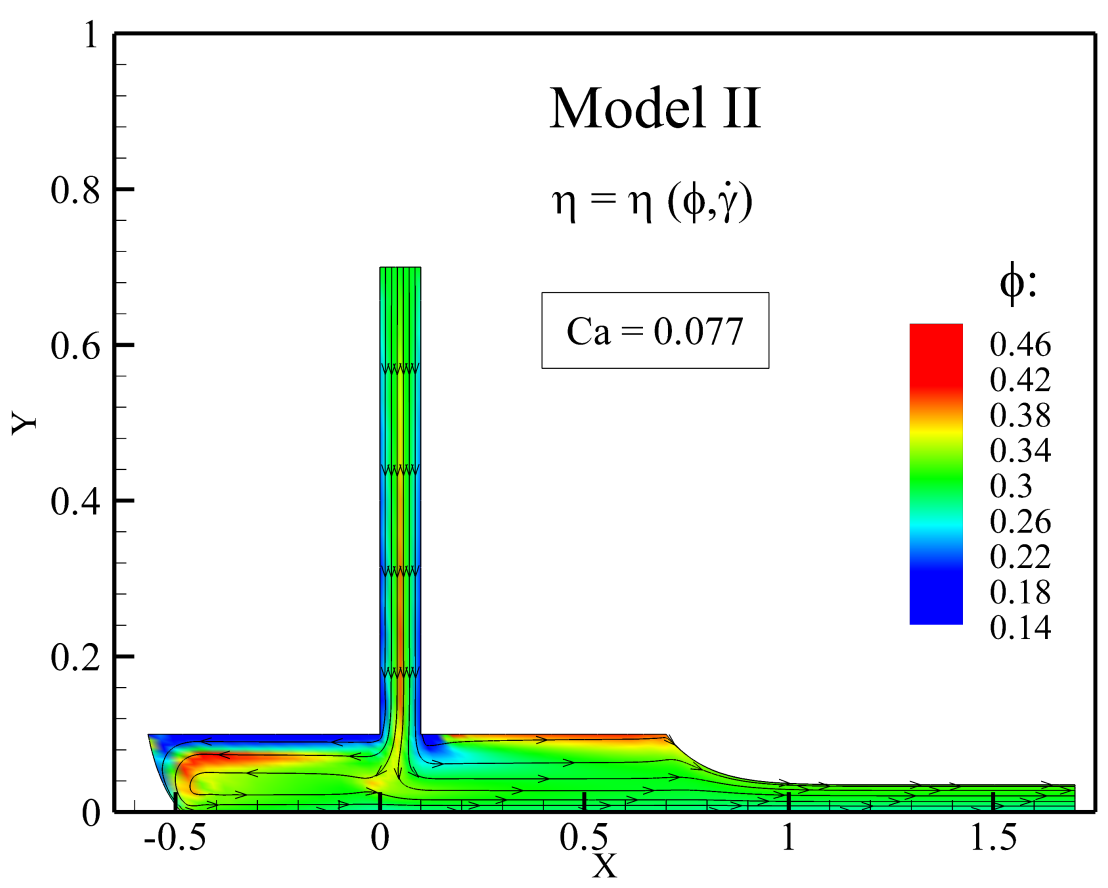

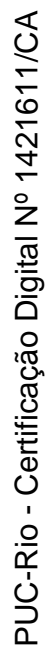

Figure 4.20: Contours of particle concentration for Capillary number 0.077 using Cross' viscosity model and migration Model II.

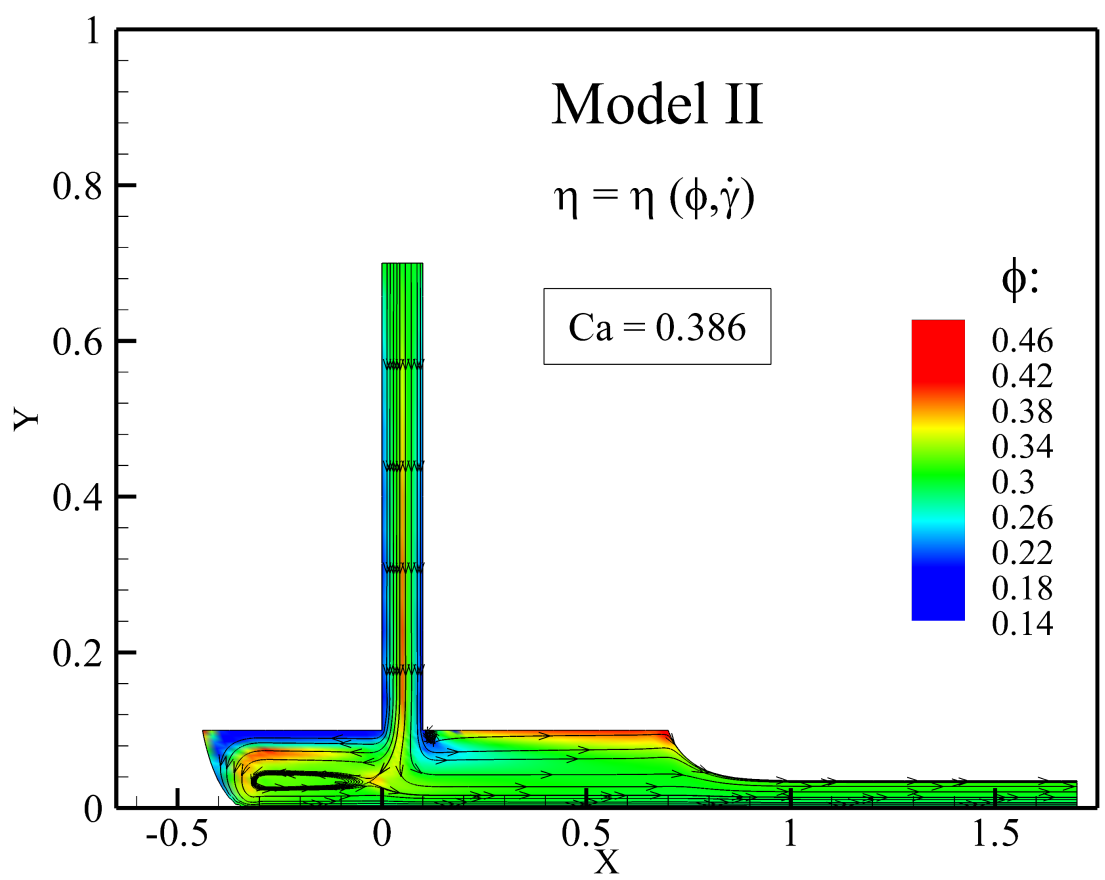

Figure 4.21: Contours of particle concentration for Capillary number 0.386 using Cross' viscosity model and migration Model II. 


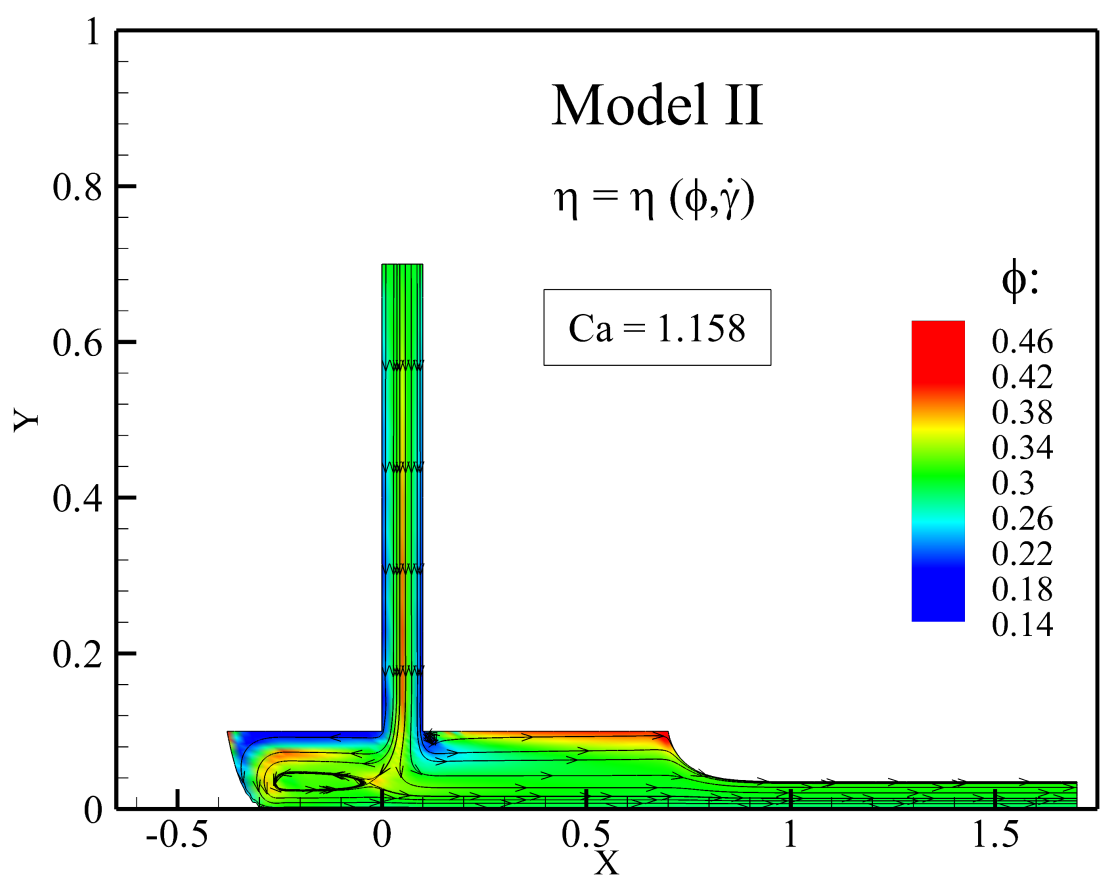

Figure 4.22: Contours of particle concentration for Capillary number 1.158 using Cross' viscosity model and migration Model II.

From those figures one can also observe that as the Capillary number increases, the upstream length and the principal radius of curvature of the gas-liquid interface decreases. The upstream length decreases due to the force balance at the free surface in this region. In other words, as the Capillary number increases, the viscous drag also rises in comparison to the same vacuum pressure applied at the meniscus so that the upstream dynamic contact line moves in the down-web direction. In addition to that, the reason for the reduction of the principal radius of curvature at the downstream gas-liquid interface relies on the balance of Poiseuille and Couette contributions in this region as depicted in Fig. 4.23. To put it another way, the flow rate under the downstream die lip can be modeled as a combination of a Couette and Poiseuille contributions:

$$
Q=V_{w} t=Q_{\text {Couette }}-Q_{\text {Poiseuille }}
$$

in which $Q_{\text {Couette }}=V H / 2$ and $Q_{\text {Poiseuille }} \propto P_{2}-P_{1}=\sigma / R$, following YoungLaplace's equation, 


$$
\Delta P_{D}=P_{2}-P_{1}=\sigma\left(\frac{1}{R_{1}}+\frac{1}{R_{2}}\right)
$$

where $\Delta P_{D}$ is the pressure difference across the downstream meniscus, $R_{1}=R$ and $R_{2}=\infty$ are the principal radii of curvature of the downstream meniscus since this interface is approximated to a cylinder. Note from Fig. 4.2:3 that, as the curvature of the deposited film is zero, there is no stress jump across the free surface, so that $P_{2}=P_{a t m}$ and hence $\Delta P_{D}=P_{a t m}-P_{1}$ at the meniscus. Moreover, $\Delta P_{D}$ must be greater than zero since the pressure on the concave side of a meniscus is always greater than the pressure on the convex side.

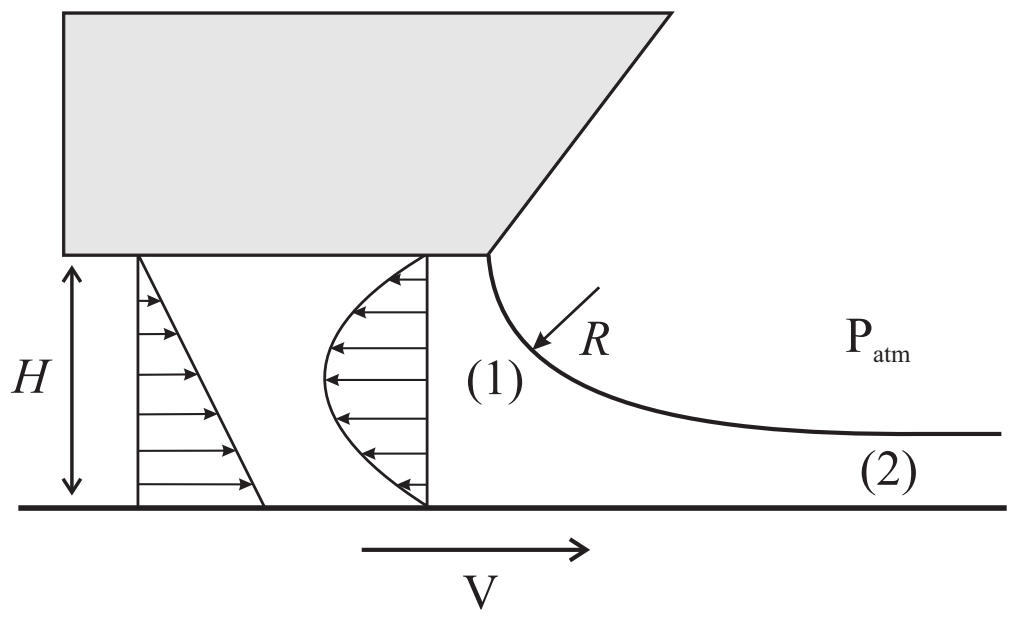

Figure 4.23: Scheme of the Couette and Poiseuille contributions at the downstream region of a slot coating flow.

Figures 4.25(b), 4.26(b) and 4.27(b) show the curvature of the streamline considering three Capillary numbers: 0.0772, 0.3860 and 1.1580, respectively, corresponding to web velocities equal to 100,500 and $1500 \mathrm{~mm} / \mathrm{s}$. Since the coating gap width is fixed for all simulations, it is convenient to introduce here a dimensionless flow rate in order to explain the relationship between the curvature of the meniscus and the Capillary number. In this manner, let a characteristic flow rate be $Q^{*}=V H$ in such a way that the dimensionless flow rate can be expressed as $\tilde{Q}=t / H$. For a fixed film thickness, if the Capillary number increases (i.e., Couette contribution), then the Poiseuille increment must increase equivalently in order to maintain a constant dimensionless flow rate. In other words, the radius of curvature of the downstream gas-liquid interface decreases and the meniscus becomes more curved.

From Figs. 4.25(a), 4.26(a) and 4.27(a) it is clear that the particles tend to accumulate at the top of the deposited film in contrast to what have been 
verified in Figures 4.53 and 4.18 , where the concentration of particles is higher at the center of the deposited film. Note that in Figs. 4.53 and 4.18 the final film thickness is $t=H / 2$ and, in this part of the results, $t=7 H / 2 \approx H / 3$. In this manner, it is possible to show that for $t=H / 2$, the flow under the downstream die is mostly determined by a Couette contribution in which the shear rate throughout this region is nearly constant and approximated by the ratio $V / H$. Consequently, particle diffusion is dominated by migrations from regions of high to low concentrations due to the terms related to $\nabla \phi$ which tends to smoothen the concentration profile. As the film thickness decreases, the adverse Poiseuille contribution increases and, as a result, the slope of the velocity profile at the downstream die wall also reduces. Therefore, a stronger gradient in shear rate is verified and particles tend to migrate towards the downstream slot die where the shear rate is small. Subsequently, those particles are convected by the flow into the deposited film region which explains the shape of the particle concentration profiles for the given three Capillary numbers examined here.

To illustrate this, Figures 4.24(a) and 4.24(b) show the contour of particle concentration for film thicknesses $t=H / 2$ and $t=7 H / 20$ considering migration Model I with a moderate Capillary number $C a=0.3860$ and viscosity model of Cross. Still regarding to 4.25(a), 4.26(a) and 4.27(a), it is possible to verify that the particle concentration profile becomes more uniform following an increase in Capillary number. In that case, as the Capillary number increases, the residence time of the particles inside the coating bead decreases and, consequently, there is not enough time for migration mechanisms to evolve in the same way as in cases of small web velocities.

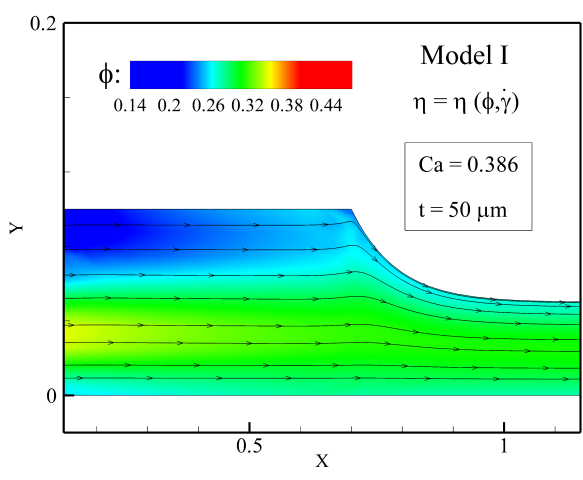

$4.24(\mathrm{a})$

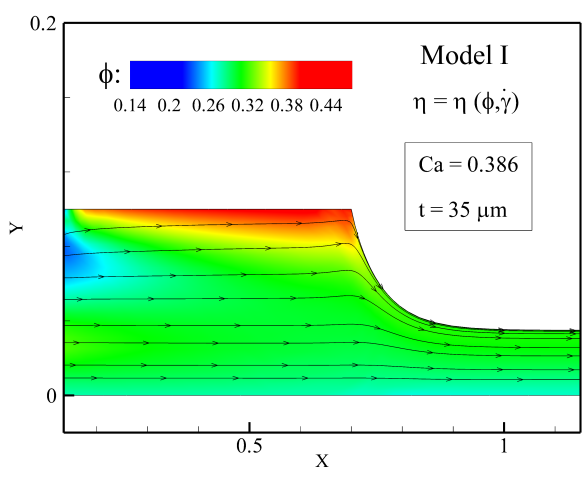

$4.24(\mathrm{~b})$

Figure 4.24: Contours of particle concentration for Capillary number $\mathrm{Ca}=$ 0.386: (a) for a final film thickness $t=H / 2$ (b) for a final film thickness $t=7 H / 20$. 


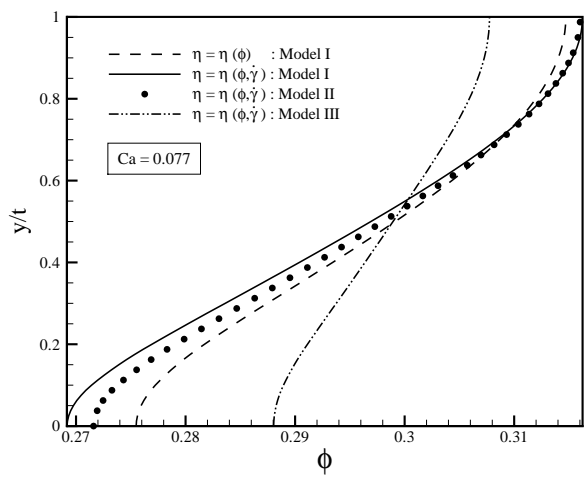

$4.25(\mathrm{a})$

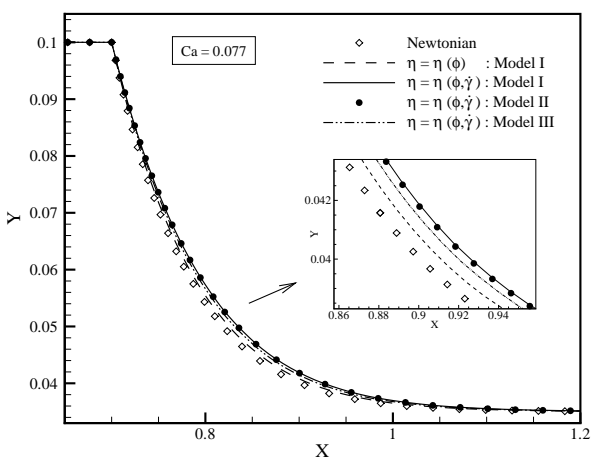

$4.25(\mathrm{~b})$

Figure 4.25: Profiles of particle concentration and upstream meniscus (a) and (b), respectively, for a Capillary number $C a=0.077$.

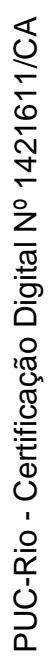

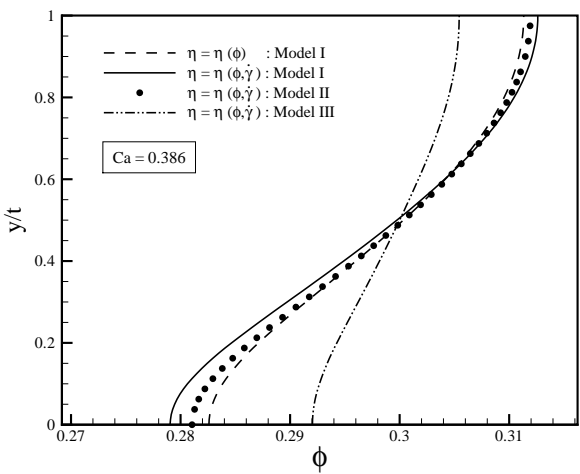

$4.26(\mathrm{a})$

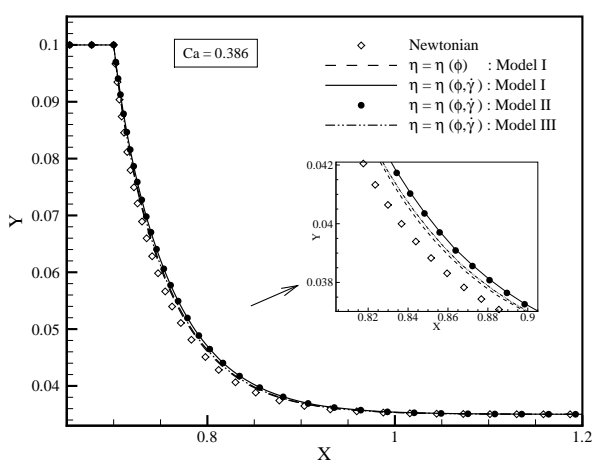

$4.26(\mathrm{~b})$

Figure 4.26: Profiles of particle concentration and upstream meniscus (a) and (b), respectively, for a Capillary number $C a=0.386$

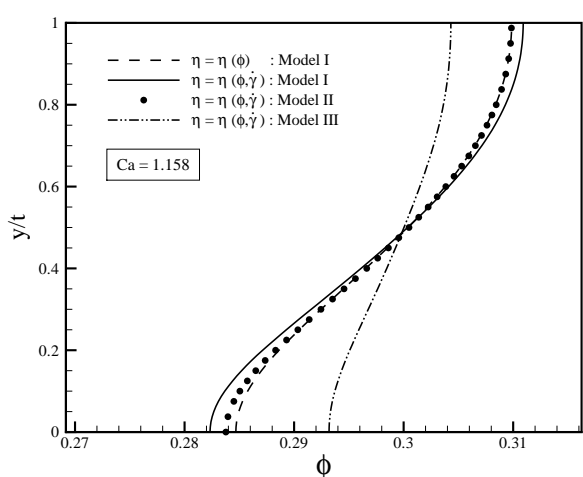

$4.27(\mathrm{a})$

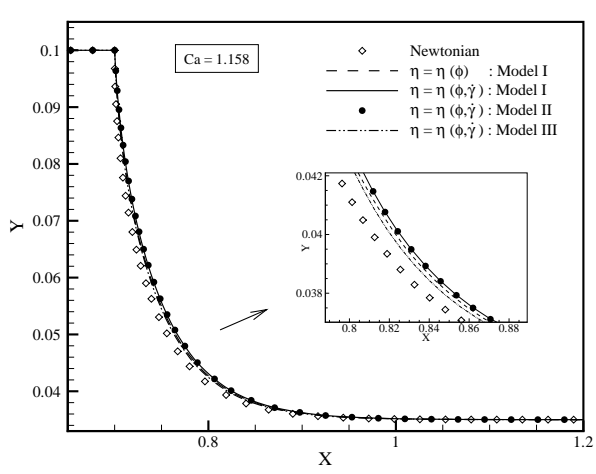

4.27 (b)

Figure 4.27: Profiles of particle concentration and upstream meniscus (a) and (b), respectively, for a Capillary number $C a=1.158$ 


\subsection{4}

\section{Low-flow limit}

In this section, the main focus is to study the effects of operating parameters and liquid properties, such as shear sensitivity, on process limits and hence on particle distribution in the coated film. In other words, a brief investigation of the coating window for a slot coating flow is performed in order to define if a specific product can be coated at a prescribed production rate. Since slot coating is classified as a pre-metered coating operation, the thickness of the deposited film is defined by the flow rate, set at the feed slot, and by the velocity of the moving substrate in such a way that the process is independent of other process variables. For instance, for a fixed substrate speed or Capillary number, the flow rate may be reduced in order to deliver a thinner coated film. Likewise, for a fixed flow rate, the web speed can be augmented to increase production rates. However, those changes are ruled out by an operating limit known as low-flow limit which is caused by a receding action of the downstream meniscus.

The low-flow limit corresponds to the maximum web speed possible at a given flow rate or the minimum film thickness possible at a given web speed at which the coating flow is steady and two-dimensional (Carvalho \& Kheshgi, 2000(1). Moreover, this stability in defined by a force balance at the downstream meniscus which is intrinsically related to the opposing contributions of Couette and Poiseuille flows beneath the downstream die. In this sense, for a given web speed or, equivalently, Capillary number, if the flow rate and thereby the film thickness decreases then the adverse contribution given by the Poiseuille flow must, on the contrary, increase since the flow rate deriving from the Couette contribution is fixed. On the other hand, for a given film thickness, if the web speed (i.e., Couette contribution) increases then the adverse Poiseuille flow must also increase in order to maintain a constant dimensionless flow rate at the downstream region. As a result, the principal radius of curvature of the downstream meniscus is reduced to increase the pressure difference across the coating bead and hence sustain a stable two-dimensional flow. However, this process cannot be performed indefinitely which means that there is a maximum pressure gradient possible above which the flow becomes instable or three-dimensional. Beyond this threshold, the upstream meniscus curves in the remaining third direction and more fluid is pumped upstream giving rise to failure mechanisms also known as ribbing and rivulets.

Following the same path, other two important limits define the coating window in a slice of the n-dimensional space of operating conditions in which the Capillary number is fixed. These limits are known as high- and low-vacuum 
limits. In this manner, if the vacuum pressure applied upstream is too high, then the liquid invades the vacuum chamber and pre-metered action is lost. On the other hand, if the upstream applied vacuum pressure is low, not enough vacuum is applied to push the upstream meniscus and hence it invades the feed slot leading to a bead breakup. Figure 4.28 gathers all three important limits that borders the coating window by defects. Note that for each Capillary number there is a critical gap-to-thickness ratio or optimum range pressure difference that must be taken into account by manufactures. As stated before, in the present work a special attention is dedicated to the low-flow limit so that the vacuum pressure is adjusted in an effort to avoid both high- and low-vacuum limits.

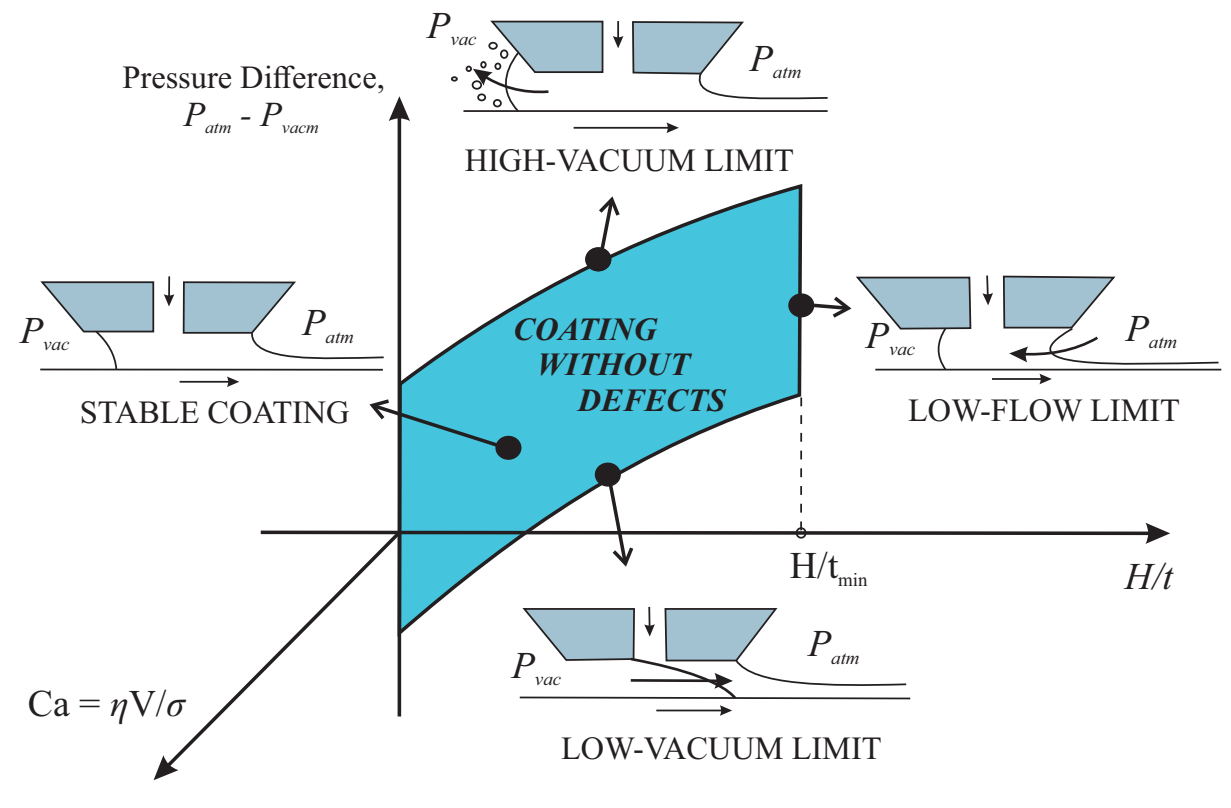

Figure 4.28: Coating window of a slot coating process as a function of the pressure difference $\left(P_{a t m}-P_{v a c}\right)$, fixed Capillary number $C a$ and gap-topthickness ratio $H / t$.

Under the light of the latter analysis, one can observe that there is a maximum pressure gradient possible at the downstream meniscus in order to guarantee a stable coating bead. In addition to that, recall that the pressure difference is ruled out by Young-Laplace's Eq. 4.9 being determined according to the principal radius of curvature of the downstream meniscus, $R$, and the interfacial tension, $\sigma$. In this sense, according to Carvalho \& Kheshgi (2000), if the shape of the free surface is assumed to be cylindrical, the smallest radius of curvature that can be obtained is 


$$
R_{\text {min }}=\frac{H-t}{2}
$$

where $H$ is the coating gap and $t$ is the film thickness. Following the works of Ruschak (1976) and Landau \& Levich (1942), the pressure gradient across the liquid/gas interface can be modeled as

$$
\Delta P=1.34 C a^{2 / 3} \frac{\sigma}{t}
$$

As a result, Eqs. 4.9, 4. 40 and 4.10 combined give birth to a relation that sets ground to the onset of low-flow limit as follows

$$
C a=0.65\left(\frac{2}{(H / t)-1}\right)^{3 / 2}
$$

Equation 4.12 is also known as the viscocapillar model and this relation is plotted in Fig. 4.29. Above the solid line, the coating bead is unstable and a steady two-dimensional flow is not possible. Although the viscocapillary model is valid only at low Capillary regimes, it is a good approximation for the threshold of coating stability and is used in this work as a reference.

The analysis of the low-flow limit is performed considering Krieger's and Cross' viscosity relations and Model I (i.e., original DFM) to predict particle migration. Models II and III are not taken into account due to numerical convergence problems as the film thickness reaches values below, approximately, $H / 3$. Under those circumstances, it was verified that curvatureinduced migration leads to very high gradients in concentration especially at regions of recirculation of the flow which possibly explains the above mentioned numerical issues.

Figure 4.2.9 depicts the low-flow limit for three different Capillary numbers: $1.158,0.2316$ and 0.0772 , respectively. Note that a Newtonian liquid behavior is approximated by considering a coating liquid in which the viscosity is a constant function of the bulk concentration. The Newtonian results deviate from the viscocapillary model for moderate to high Capillary numbers. In that case, for Capillary numbers $C a=1.158$ and $C a=0.2316$ this discrepancy can be explained by the fact the viscocapillary model is only valid at low Capillary numbers which is in agreement with the works presented by Carvalho \& Kheshgi (2000) and Romero et al. (20104). Equally important, note that for 
$C a=0.0772$, the Newtonian result recovers the viscocapillary model, as expected. Still in the same track, one shall observe that as particle migration is considered, the zone of stability of the coating flow is broadened. In fact, for shear sensitive liquids, where a shear thinning behavior is dominant, the minimum film thickness possible is even smaller for a given web speed.

From Fig. 4.2.9 one can also observe that the discrepancy between Cross' model and the Newtonian result apparently decreases as Capillary number rises. For instance, the absolute difference is around 1.2 for Capillary numbers 0.0772 and 0.2316 and 0.712 for $C a=1.158$. In addition, considering Krieger's viscosity model it is possible to detect the same trend in which the absolute differences are: $0.322,0.37$ and 0.40 for Capillary numbers $0.077,0.2316$ and 1.158 .

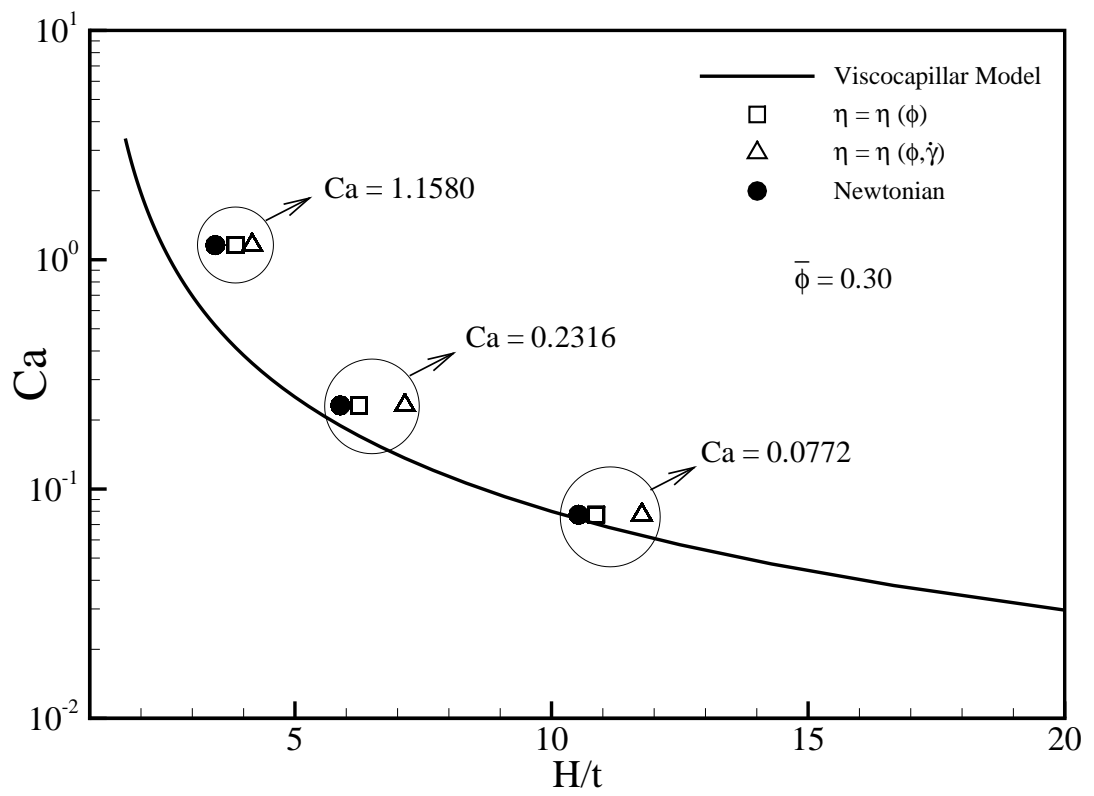

Figure 4.29: Low-flow limit predictions by: viscocapillary model and viscosity models of Krieger and Cross considering migration Model I.

Figure 4.30 shows the meniscus configuration at the critical gap-tofilm thicknesses considering all viscosity models a fixed Capillary number. Again, note that, as particle migration is taken into account, the region of stability is broadened. Moreover, if the suspension is shear sensitive, smaller film thicknesses can be obtained for the same production rate or web velocity.

Figures 4.31 and 4.32 show the contour map of concentration field and particle concentration profiles at the final deposited film for $C a=0.2316$ and $t \approx H / 7$. Under those circumstances, a recirculation appears under the downstream die and a considerable amount of particles tend to be trapped inside the vortex which is in agreement with the Newtonian results for coating 
simulations for $t<H / 3$ (Silva, 2013). In that case, the adverse flow due to the pressure difference across the downstream meniscus creates a layer of maximum negative velocity and thereby vanishing shear rate close to the die lip towards which the particles migrate by means of shear-induced particle migration. In this way, particles are convected to the film region by viscous drag which explains the shape of particle concentration profile at the deposited film in Fig. 1.32. Again, the concentration of particles at the top of the film is greater for a shear sensitive liquid in comparison with a concentration-dependant one. Considering that the dimensionless flow rate is, approximately, constant for both viscosity models, in order to maintain the same stress at the wall, if the the viscosity is lower, then higher gradients in shear rate are generated leading to a stronger flux of particles towards regions of low shear rates such as the layer of maximum negative velocity under the downstream die.

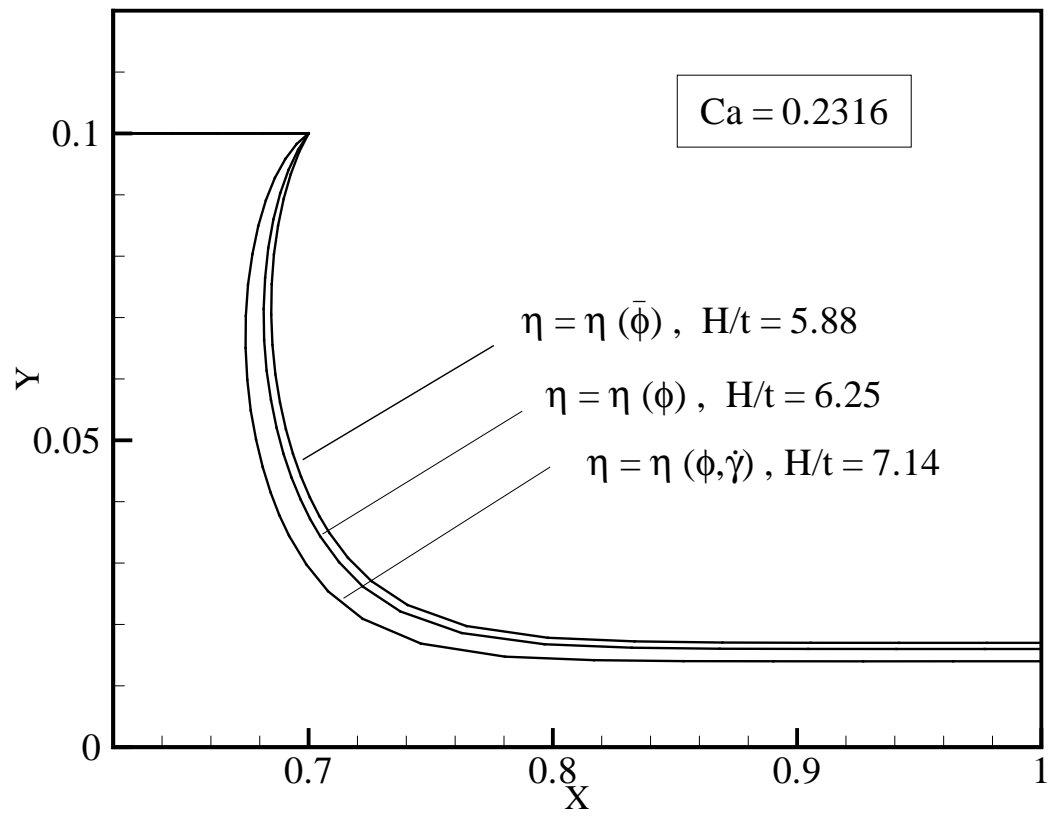

Figure 4.30: Downstream free surface at different gap-to-thickness ratio and Capillary numbers considering concentration-dependent only viscosity. 


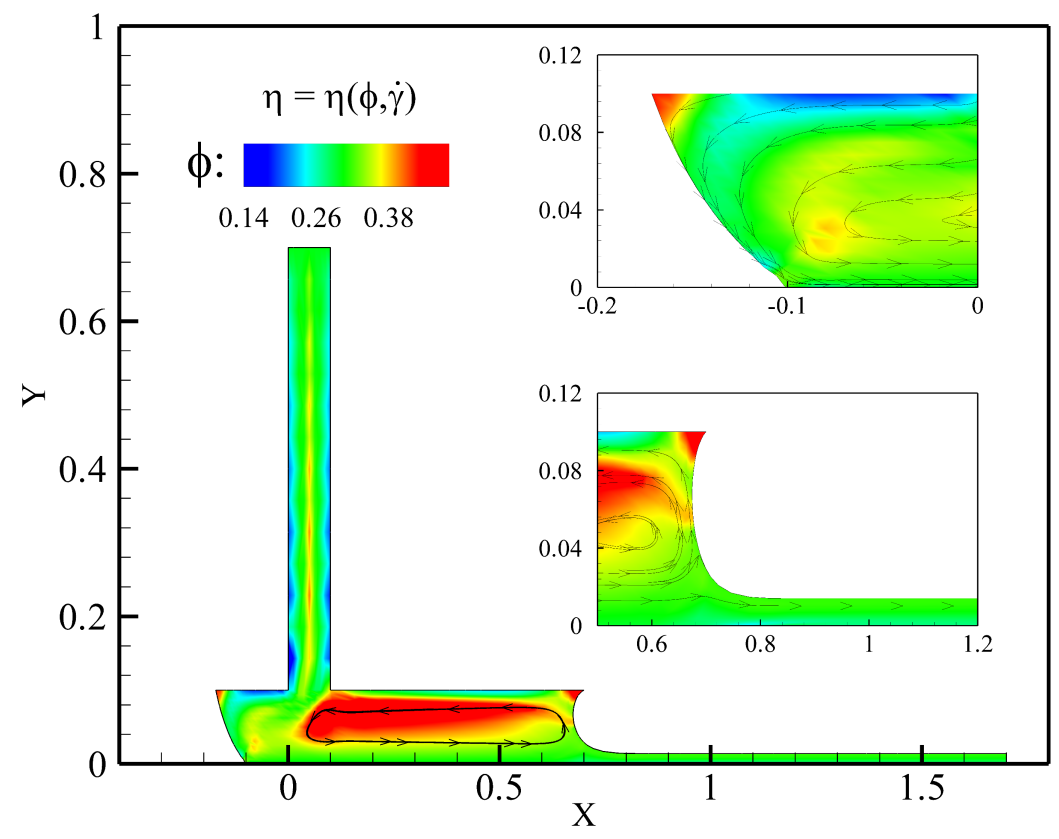

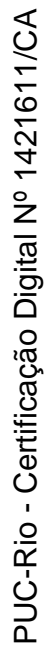

Figure 4.31: Contour map of concentration field considering viscosity model of Cross' considering a fixed Capillary number $C a=0.2316$.

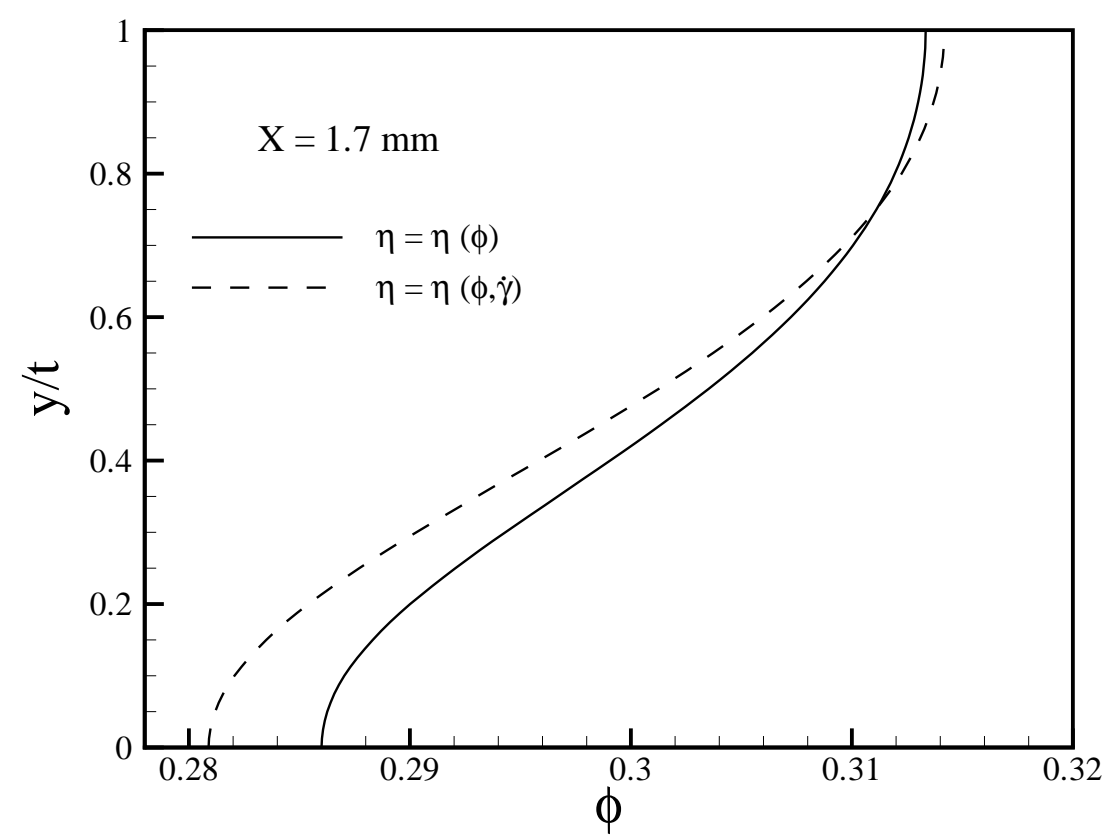

Figure 4.32: Particle concentration profiles comparing both viscosity models considering a fixed Capillary number $C a=0.2316$. 


\section{5}

\section{Final remarks}

In this work we investigated numerically the effects of liquid rheological properties (e.g., pseudo-plastic behavior) on process limits and particle distribution on a typical slot coating flow. In order to capture this non-Newtonian behavior the viscosity model of Cross ([1970) was chosen and the values of the material constants follow experimental predictions presented by Choi \& Krieger (1986). On the other hand, in an effort to include particle migration mechanisms, three migration models were studied, namely: Model I, Diffusive Flux Model (DFM) proposed by Phillips et al. ([.992) in which shear-induced particle migration is the main source of flux; Model II, which is an extension of Model I that also includes curvature-induced migration; and, finally, Model III, which in turn is an adaptation of Model II with volume-fraction-dependent parameters. With all the equations posed, the whole set of non-linear partial differential equations were solved numerically by using the GFEM coupled with Newton's method. However, numerical issues were encountered due to the singularity of the DFM in regions where local shear rate approaches zero leading to convergence problems. In order to overcome this adversity, we employed a modified version of the non-local stress contribution to the shear rate in which $\dot{\gamma}=\dot{\gamma}_{\delta}$ when $\dot{\gamma} \rightarrow 0$ (Miller \& Morris, 20106; Ahmed \& Singh, 2011).

The results of this work were divided in three main blocks regarding the study of a typical slot coating flow. First, we analyzed the influence of considering or not a shear sensitive viscosity model in cases where particle size is small enough so that colloidal forces compete with macroscopic forces of the flow yielding a shear thinning behavior. In this way, for a fixed dimensionless flow rate or final film thickness, we verified that when this pseudo-plastic behavior is taken into account, the size of the upstream region of the slot coating flow is augmented in comparison to the case where a concentrationdependent viscosity model is employed. Also, given that the flow rate and hence the shear stress at the wall is fixed for both cases, a reduction in local viscosity generates an increase in shear rate so that the flux of particles towards regions of low rates of shear is intensified. For instance, in the case where $t=H / 2$ the particles tend to be concentrated at the center of the coated film. 
Also, an examination of all migration models is performed in this part of the results. To this end, with regards to Model II, we showed that as curvatureinduced migration is considered, more particles tend to migrate towards regions opposite to curved streamlines like those ones close to the upstream static contact line. In addition to that, we also have shown that Model III (i.e., with concentration-dependent diffusion parameters) delivers a more uniform concentration field. Since the ratio $k_{c} / k_{\eta}$ depends on local concentration, and this dependency is explicit on $k_{c}=k_{c}(\phi)$, the migration mechanism due to gradients in viscosity dominates those related to gradients in shear rate and to the curvature of the streamlines. This mechanism acts on an opposite direction of the latter two mentioned fluxes delivering a more uniform concentration profile.

The second block of results explores the effects of the Capillary number on particle concentration profiles at the coated film and on the shape of the downstream meniscus for a fixed film thickness. First of all, in this part of the results we verified that for a film thickness $t \approx H / 3$, the velocity profile under the downstream die is such that the shear rate close at the wall is lower and a shear-induced particle migration towards this area is pronounced. As a result, this agglomerate of particles is convected by the flow to the film formation region so that the particles tend to accumulate at the top of the coated film. Additionally, for a fixed vacuum pressure, we showed that as the Capillary number increases, the upstream dynamic contact line moves in the down-web direction and the downstream meniscus becomes more curved. This latter phenomenon is due to a receding action of the gas-liquid interface to cope with an increase of the Couette flow rate under the downstream die. Again, since the dimensionless flow rate is constant, an increment in Couette contribution must be followed by an equivalent increase in adverse Poiseuille contribution that is made possible by a reduction of the radius of curvature of the downstream meniscus.

Finally, the third block of results focus on a well-established operating limit for a slot coating application also known as low-flow limit. In this sense, we presented the minimum film thickness or flow rate possible given a fixed web speed or Capillary number. With this in mind, a comparison between the numerical predictions of the onset of low-flow limit considering both viscosity relations and the viscocapillary model was performed. In the latter analysis we verified that the region of stability is broadened as particle migration mechanisms are taken into account. Moreover, it was detected that even smaller film thicknesses can be obtained if the suspension is considered shear sensitive. A further investigation is made in order to study the shape of 
the downstream meniscus and particle concentration profile at the deposited film. In this sense, it follows that for a given viscosity model, the critical gapto-thickness ration varies with the Capillary number so that the downstream meniscus becomes more curved for higher values of web velocity. Alternatively, for a fixed Capillary number, we verified a similar trend as viscosity model was changed. For a Capillary number $C a=0.2316$, the meniscus of a shear sensitive suspension becomes more curved than the one of a concentrationdependent viscosity yielding a thinner final film thickness. With regards to particle concentration distribution, one shall observe that, for $t=H / 7$, the particles tend to be trapped inside the vortex or recirculation region just below the downstream die. In that case, the adverse Poiseuille flow generates a layer of vanishing shear rate at the region of maximum negative velocity towards which the particles are driven due to shear-induced particle migration. Also, as a final analysis, we compared particle concentration profiles at the deposited film considering the viscosity models of Cross and Krieger, respectively. Again, as the dimensionless flow rate is the same for both cases, if the viscosity at the downstream die decreases, then gradients in shear rate are intensified driving more particles to regions of lower shear rates. As a result, the concentration of particles at the top of the deposited film is greater than the case in which the viscosity is a function of the local concentration only, i.e., neglecting shear rate dependency.

\section{1}

\section{Future Works}

As suggestions of future works one shall consider:

- Perform experiments in order to better understand the physics of the problem and confirm the validity of the numerical results presented in this work. In this manner, confocal microscopy could be a good experimental technique to investigate particle distribution in the final film thickness. Also, experimental results are important in an effort to fit the phenomenological parameters of the DFM in accordance to the complexities of a typical slot coating flow.

- Improve the numerical formulation in order to overcome numerical issues related to convergence problems when curvature-induced migration is taken into account in simulations where the final film thicknesses is lower than $H / 3$.

- Expand the model to consider effects of sedimentation for colloidal suspensions where inter-particle forces may assume an important role. 
In addition to that, include effects of drying processes to predict and understand the final particle distribution on the coated product.

- In addition, consider the particle orientation with respect to the flow in cases of non-spherical particles. For instance, Siqueira (2016) employed a modified particle conformation tensor for the orientation of ellipsoidal particles in complex flows. The same model might be applied but, now, considering the effects of a shear-sensitive viscosity relation. 


\section{Bibliography}

Abbott, J. R., Tetlow, N., Graham, A. L., Altobelli, S. A., Fukushima, E., Mondy, L. A., \& Stephens, S. (1991). Experimental observations of particle migration in concentrated suspensions: Couette flow. Journal of Rheology, 35, 773-795.

Ahmed, G. M. Y. \& Singh, A. (2011). Numerical simulation of particle migration in asymmetric bifurcation channel. Journal of Non-Newtonian Fluid Mechanics, 166, 42-51.

Araújo, S. B. (2014). Slot coating of particle suspension. Master's thesis, PUC-Rio, Rio de Janeiro, RJ-Brazil.

Aris, R. (1962). Vectors, tensors and the basic equations of fluid mechanics. Dover Publications, INC.

Babuska, I. (1973). The finite element method with lagrangean multipliers. Numer. Math., 20, 179-192.

Barnes, H. A., Hutton, J. F., \& Walters, K. (1989). An Introduction to Rheology. Elsevier Science B. V.

Batchelor, G. K. (1967). An introduction to fluid dynamics. Cambridge University Press.

Batchelor, G. K. (1977). The effect of brownian motion on bulk stress in a suspension of spherical-particles. Journal of Fuid Mechanics, 83, 97-117.

Batchelor, G. K. \& Green, J. T. (1972). Hydrodynamic interaction of two small freely moving spheres in a linear flow field. Journal of Fluid Mechanics.

Beguin, A. E. (1954). Method of coating strip material. U.S. Patent No. 2,681,294.

Benjamin, D. F. (1994). Roll Coating Flows and Multiple Roll Systems. PhD thesis, University of Minnesota, Minneapolis, MN-USA.

Bird, R. B., Armstrong, R. C., \& Hasser, O. (1987). Dynamic of polymeric liquids. Wiley. 
Bossis, G. \& Brady, J. F. (1989). The rheology of browian suspensions. Journal of Chemical Physics, 91, 1866-1874.

Brown, R. (1827). On the particles contained in the pollen of plants and on the general existence of active molecules in organic and inorganic bodies. Not Published.

Carreau, P. J. (1972). Rheological equations from molecular network theories. Transactions of the Society of Rheology, 16, 99-127.

Carvalho, M. S. (1996). Roll Coating Flows in Rigid and Deformable gaps. $\mathrm{PhD}$ thesis, University of Minnesota.

Carvalho, M. S. \& Kheshgi, H. S. (2000). Low-flow limit in slot coating: Theory and experiments. AIChe Journal, 46, 1907-1917.

Chen, L. B., Ackerson, B. J., \& Zukoski, C. F. (1994). Rheological consequences of microstructural transitions in colloidal crystals. Journal of Rheology, 38, 193-216.

Cheng, X., McCoy, J. H., Israelachvili, J. N., \& Cohen, I. (2011). Imaging the microscopic structure of shear thinning and thickening colloidal suspensions. Science, 333, 1276-1279.

Choi, G. N. \& Krieger, I. M. (1986). Rheological studies on sterically stabilized model dispersions of uniform colloidal spheres. Journal of Colloid and Interface Science, 113, 101-113.

Chow, A. W., Sinton, S. W., \& Iwamiya, J. H. (1994). Shear-induced particle migration in couette and parallel-plate viscometers: Nmr imaging and stress measurements. Physics of Fluids, 6, 2561-2575.

Christodolou, K. N. \& Scriven, L. E. (1992). Discretization of free surface flows and other moving boundary problems. Journal of Computational Physics, 99, 39-55.

Cross, M. M. (1970). Kinetic interpretation of non-newtonian flow. Journal of Colloid and Interface Science, 33, 30-35.

Cunha, F. R. \& Hinch, E. J. (1996). Shear-induced dispersion in a dilute suspension of rough spheres. Journal of Fluid Mechanics, 309, 211-223.

Dbouk, T. (2011). Rheology of concentrated suspensions and shear-induced migration. PhD thesis, Laboratoire de Physique de la Matière condensèe Universitè de Nice-Sophia Anipolis. 
de Almeida, V. F. (1995). Gas-liquid counterflow through constricted passages. $\mathrm{PhD}$ thesis, University of Minnesota, Minneapolis, MN-USA.

de Almeida, V. F., Chapman, A. M., \& Derby, J. J. (1999). On equilibration and sparse factorization of matrices arising in finite element solutions of partial differential equations. Numerical Methods for Partial Differential Equations.

de Kruif, C. G., van Iersel, M. F., Vrij, A., \& Russel, W. B. (1985). Hard sphere colloidal dispesions: Viscosity as a function of shear rate and volume fraction. The Journal of Chemical Physics, 83, 4717-4725.

Deryagin, B. M. \& Levi, S. M. (1964). Film Coating Theory. Focal Press.

Donea, J. \& Huerta, A. (2003). Finite Element Method for Flow Problems. John Wiley \& Sons.

Eckestein, E. C., Bailey, D. G., \& Shapiro, A. H. (1977). Self-diffusion of particles in shear flow of a suspension. Journal of Fluid Mechanics, 79, 191-208.

Eilers, H. (1941). Die viskositat von emulsionen hochviskoser stoffe als funktion der konzentration. Kolloid Z., 97, 313-321.

Einstein, A. (1906). Eine neue bestimmung der molekuledimensionen. Annalen der Physik, 19, 289-306.

Einstein, A. (1911). Berichtigung zu meiner arbeit: Eine neue bestimmung der molekuledimensionen. Annalen der Physik, 34, 591-592.

Fang, Z. \& Phan-Thien, N. (1995). Numerical simulation of particle migration in concentrated suspensions by a finite volume method. Journal of NonNewtonian Fluid Mechanics, 58, 68-81.

Foss, D. R. \& Brady, J. F. (2000). Structure, diffusion and rheology of brownian suspenions by stokesian dynamics simulation. Journal of Fluid Mechanics, 407, 167-200.

Franca, L. P. \& Frey, S. L. (1991). Stabilized finite element methods: Ii the incompressible navier-stokes equations. Computer Methods in Applied Mechanics and Engineering, 99, 209-233.

Gadala-Maria, F. \& Acrivos, A. (1980). Shear-induced structure in a concentrated suspension of solid spheres. Journal of Rheology, 24, 799-814. 
Gates, I. D. (1996). Stability analysis of slot coating flow. AIChE Meeting.

Gontijo, R. G. (2013). Micromechanics and Microhydrodynamics of Magnitic Suspensions. PhD thesis, University of Brasilia, Brasilia, Brazil.

Graham, A. L., Mammoli, A. A., \& Bush, M. B. (1998). Effects of demixing on suspension rheometry. Rheologica Acta, 37(2), 139-150.

Gresho, P. M. \& Sani, R. L. (1998). Incompressible Flows and the Finite Element Method: Advection-Diffusion and Isothermal Laminar Flow. John Wiley \& Sons.

Guth, E. \& Simha, R. (1936). Untersuchungen uber die viskositat von suspensionen und losungen. 3. uber die viskositat von kugelsuspensionen. Kolloid Z., 74, 266-275.

Higgins, B. G. \& Scriven, L. E. (1980). Capillary pressure and viscous pressure drop set bounds on coating bead operability. Chemical Engineering Science, $35,673-682$.

Hill, R. J., Koch, D. L., \& Ladd, A. J. C. (2001). The first effect of fluid inertia on flows in ordered and random arrays of spheres. Journal of Fluid Mechanics, 448, 213-241.

Hinch, E. J. (2011). The measurement of suspension rheology. Journal of Fluid Mechanics, 686, 1-4.

Hoffman, R. L. (1972). Discontinuous and dilatant viscosity behavior in concentrated suspensions. i. observation of a flow instability. Transactions of the Society of Rheology, 16.

Huh \& Scriven (1971). Hydrodynamic model of steady movement of a solid/liquid/fluid contact line. Journal of Colloid and Interface Science, $1,85-101$.

Husband, D. M., Mondy, L. A., Ganani, E., \& Graham, A. L. (1994). Direct measurements of shear-induced particle migration in suspensions of bimodal spheres. Rheologica Acta, 33, 185-192.

Jeffery, G. B. (1922). The motion of ellipsoidal particles immersed ia a viscous fluid. Proceedings of the Royal Society of London, Series A, 1, 161-179.

Jenkins, J. T. \& McTigue, D. F. (1990). Transport processes in concentrated suspensions: The role of particle fluctuations. Two Phase Flows and Waves, $26,70-79$. 
Karnis, A., Goldsmith, H. L., \& Mason, S. G. (1966). The kinetics of flowing dispersions i. concetrated suspensions of rigid particles. Journal of Colloid and Interface Science, 22, 531-553.

Kim, J. M., Lee, S. G., \& Kim, C. (2008). Numerical simulations of particle migration in suspension flows: Frame-invariant formulation of curvatureinduced migration. AIChE Journal, 150, 162-176.

Krieger, I. M. (1972). Rheology of monodisperse latices. Advances in Colloid and Interface Science, 3, 111-136.

Krieger, I. M. \& Dougherty, T. J. (1959). A mechanism for non-newtonian flow in suspensions of rigid spheres. Transactions of the Society of Rheology, $3,137-152$.

Krishnan, G. P., Beimfohr, S., \& Leighton, D. T. (1995). Shear-induced radial segragation in bidisperse suspensions. Journal of Fuid Mechanics, 321, 371393.

Landau, L. \& Levich, B. (1942). Dragging of a liquid by a moving plate. Acta Physicochimica URSS, 17, 42-54.

Larson, R. G. (1999). The Structure and Rheology of Complex Fuids. Oxford University Press.

Laun, H. M. (1984). Rheological properties of aqueous polymer dispersions. Macromolecular Materials and Engineering, 123, 335-359.

Lee, K. Y. \& Liu, T. J. (1992). Minimum web thickness in extrusion slot coating. Chemical Engineering Science, 47, 1703-1713.

Leighton, D. \& Acrivos, A. (1987a). Measurement of shear-induced self-difusion in concentrated suspensions of spheres. Journal of Fluid Mechanics, 177, $109-131$.

Leighton, D. \& Acrivos, A. (1987b). The shear-induced migration of particles in concentrated suspensions. Journal of Fluid Mechanics, 181, 415-439.

Lin, C. F., Wong, D. S. H., Liu, T. J., \& Wu, P. Y. (2010). Operating windows of slot die coating: Comparison of theoretical predictions with experimental observations. Advances in Polymer Technology, 29, 31-44.

Loimer, T., Nir, A., \& Semiat, R. (2002). Shear-induced corrugation of free interfaces in concentrated suspensions. Journal of Fluid Mechanics, 102, $115-134$. 
Macosko, C. W. (1994). Rheology: Principles, Measurements and Applications. Wiley-VCH, Inc.

Majors, P. D., Gilver, R. C., \& Fukushima, E. (1989). Velocity and concentration measurements in multiphase flows by nmr. Journal of Magnetic Resonance, 85, 235-243.

Maron, S. H. \& Pierce, P. E. (1956). Application of ree-eyring generalized flow theory to suspensions of spherical particles. Journal of Colloid and Interface Science, 11, 80-95.

Maza, D. \& Carvalho, M. S. (2015). Transient response of two-layer slot coating flows to periodic disturbances. AIChe Journal, 61, 1699-1707.

Miller, R. M. \& Morris, J. F. (2006). Normal stress-driven migration and axial development in pressure-driven flow of concentrated suspensions. Journal of Non-Newtonian Fluid Mechanics, 135, 149-165.

Min, K. H. \& Kim, C. (2010). Simulation of particle migration in free-surface flows. AIChE Journal, 56, 2539-2550.

Mooney, M. (1951). The viscosity of a concentrated suspension of spherical particles. Journal of Colloid Science, 6, 162-170.

Morris, J. F. \& Boulay, F. (1999). Curviliniar flows of noncolloidal suspensions: The role of normal stresses. Journal of Rheology, 43, 1213-1237.

Nott, P. R. \& Brady, J. F. (1994). Pressure-driven flow of suspensions: simulation and theory. Journal of Fluid Mechanics, 275, 157-199.

Pasquali, M. (2000). Polymer Molecules in free surface coating flows. PhD thesis, University of Minnesota, Minneapolis, MN-USA.

Pasquali, M. \& Scriven, L. E. (2002). Free surface flows of polymer solutions with models based on the conformation tensor. Journal of Non-Newtonian Fluid Mechanics, 108, 363-409.

Phillips, R. J., Armstrong, R. C., \& Brown, R. A. (1992). A constitutive equation for concentrated suspensions that accounts for shear-induced particle migration. Physics of Fluids A, 4, 30-40.

Quemada, D. (1977). Rheology of concentrated disperse systems and minimum energy-dissipation principle. Rheologica Acta, 16, 82-94. 
Rebouças, R. B., Siqueira, I. R., de Souza Mendes, P. R., \& Carvalho, M. S. (2016). On the pressure-driven flow of suspensions: particle migration in shear sensitive liquids. Journal of Non-Newtonian Fluid Mechanics, 234, $178-187$.

Romero, O. J. \& Carvalho, M. S. (2008). Response of slot coating flows to periodic disturbances. Chemical Engineering Science, 63, 2161-2173.

Romero, O. J., Suszynski, W. J., Scriven, L. E., \& Carvalho, M. S. (2004). Low-flow limit in slot coating of dilute solutions of high molecular weight polymer. Journal of Non-Newtonian Fluid Mechanics, 118, 137-156.

Ruschak, K. J. (1976). Limiting flow in a pre-metered coating device. Chemical Engineering Science, 31, 1057-1060.

Santos, J. M. (1991). Two-Phase Cocurrent Downflow through Constricted Passages. PhD thesis, University of Minnesota, Minneapolis, MN-USA.

Sartor, L. (1990). Slot Coating: Fluid Mechanics and Die Design. PhD thesis, University of Minnesota.

Sato, T. (1995). Rheology of suspensions. Journal of Coatings Technology, 67, 69-79.

Sato, T. (1997). Simulating the rheology of dense colloidal suspensions using dissipative particle dynamics. Physical Review E, 55, 3124-3133.

Seto, R., Mari, R., Morris, J. F., \& Denn, M. M. (2013). Discontinuous shear thickning of frictional hard-sphere suspensions. Physics Review Letters, 111, 218301.

Shewan, H. M. \& Stokes, J. R. (2015). Analytically predicting the viscosity of hard sphere suspensions from the particle size distribution. Journal of Non-Newtonian Fluid Mechanics, 222, 72-81.

Silliman, W. J. (1979). Viscous Film Flows with Contact Lines: Finite Element Simulation, a Basis for Stability Assessment and Design Optimization. $\mathrm{PhD}$ thesis, University of Minnesota, Minneapolis, MN-USA.

Silva, L. D. V. (2013). Slot coating of particle suspension. Master's thesis, PUC-Rio, Rio de Janeiro, RJ-Brazil.

Simha, R. (1949). Effect of concentration on the viscosity of dilute solutions. Journal of Research of the National Bureau of Standards, 42, 409-418. 
Singh, A., Nir, A., \& Semiat, R. (2006). Free-surface flow of concentrated suspensions. International Journal of Multiphase Flow, 32, 775-790.

Siqueira, I. R. (2016). Slot coating flows of elongated particle suspensions. Master's thesis, PUC-Rio, Rio de Janeiro, RJ-Brazil.

Snook, B. B., Butler, J. E., \& Guazzelli, E. (2016). Dynamics of shear-induced migration of spherical particles in oscillatory pipe flow. Journal of Fluid Mechanics, 786, 128-153.

Stickel, J. J. \& Powell, R. L. (2005). Fluid mechanics and rheology of dense suspensions. Annual Review of Fluid Mechanics, 37, 129-149.

Subia, S. R., Ingber, M. S., Mondy, L. A., Altobelli, S. A., \& Graham, A. L. (1998). Modelling of concentrated suspensions using a continuum constitutive equation. Journal of Fluid Mechanics, 373, 193-219.

Szad, M. J., Salamon, T. R., Liu, A. W., Bornside, D. E., Armstrong, R. C., \& Brown, R. A. (1995). A new mixed finite element method for viscoelastic flows governed by differential constitutive equations. Journal of NonNewtonian Fuid Mechanics, 59, 215-243.

Tetlow, N., Graham, A. L., Ingber, M. S., Subia, S. R., Mondy, L. A., \& Altobelli, S. A. (1998). Particle migration in a couette apparatus: experimental and modeling. Journal of Rheology, 42(2), 307-327.

Timberlake, B. D. \& Morris, J. F. (2002). Concentration band dynamics in free-surface couette flow of a suspension. Physics of Fluids, 14, 1580-1589.

Tirumkudulu, M., Mileo, A., \& Acrivos, A. (1999). Particle segregation in monodisperse sheared suspensions. Physics of Fluids, 11, 507-509.

Tirumkudulu, M., Mileo, A., \& Acrivos, A. (2000). Particle segregation in monodisperse sheared suspensions in a partially filled rotating horizontal cylinder. Physics of Fluids, 12, 1615-1618.

Vand, V. (1947). Viscosity of solutions and suspensions. Journal of Physical Chemistry, 52, 277-299.

Wyart, M. \& Cates, M. E. (2014). Discontinuous shear thickning without inertia in dense non-brownian suspensions. Physics Review Letters, 111, 098302.

Zhang, K. \& Acrivos, A. (1994). Viscous resuspension in fully developed laminar pipe flows. International Journal of Multiphase Flow, 20, 579-591. 